\title{
DISSERTATION
}

\section{On the kernel of the reciprocity map of varieties over local fields}

\author{
ZUR ERLANGUNG DES DOKTORGRADES DER \\ NATURWISSENSCHAFTEN (DR. RER. NAT.) \\ AN DER FAKULTÄT FÜR MATHEMATIK \\ DER UNIVERSITÄT REGENSBURG
}

\author{
vorgelegt von \\ Patrick Forré \\ aus Engelskirchen
}

16. Juni 2011

\author{
betreut durch \\ Prof. Dr. Uwe Jannsen
}


Promotionsgesuch eingereicht am: 16. Juni 2011

Die Arbeit wurde angeleitet von: Prof. Dr. Uwe Jannsen

Prüfungsausschuss:

Vorsitzender:

Prof. Dr. Helmut Abels

1. Gutachter:

Prof. Dr. Uwe Jannsen

2. Gutachter:

Prof. Dr. Alexander Schmidt, Heidelberg

weiterer Prüfer:

Prof. Dr. Bernd Ammann

Ersatzprüfer:

Prof. Dr. Klaus Künnemann 


\section{Introduction}

One main problem in arithmetic geometry is the computation of the étale fundamental group $\pi_{1}(X, \bar{x})$ of a scheme $X$, classifying its finite étale covers. Because this problem is too hard to solve, in class field theory one restricts to the comparison of the abelianized fundamental group $\pi_{1}^{\mathrm{ab}}(X)$, classifying finite abelian étale covers of $X$, with a suitable so-called class group $C_{X}$, like in the classical case of a local or global field. The class group usually is linked to more local data and is supposed to be more easy to handle. The comparison is done via a homomorphism

$$
\rho^{X}: C_{X} \rightarrow \pi_{1}^{\mathrm{ab}}(X)
$$

which is called reciprocity map. In the best case $\rho^{X}$ would be an isomorphism. But in general this is not true. So the main task of class field theory will be to determine the cokernel and the kernel of $\rho^{X}$.

In this thesis we will consider class field theory for higher dimensional varieties $X$ over local fields $K$ following the ideas of S. Bloch, K. Kato, S. Saito and U. Jannsen (cf. [Blo81], [KS83, [Sai85a], [JS03]). In this setting the class group for a proper variety is given by

$$
\mathrm{SK}_{1}(X):=\operatorname{coker}\left(\bigoplus_{y \in X_{1}} K_{2}(\kappa(y)) \stackrel{\partial}{\rightarrow} \bigoplus_{x \in X_{0}} \kappa(x)^{\times}\right),
$$

where $\partial$ is induced by the boundary maps coming from algebraic $K$-theory and where $X_{a}$ is the set of $a$-dimensional points. The reciprocity map

$$
\rho^{X}: \mathrm{SK}_{1}(X) \rightarrow \pi_{1}^{\mathrm{ab}}(X)
$$

then is defined to be the sum of local reciprocity maps $\rho_{x}: \kappa(x)^{\times} \rightarrow \mathrm{Gal}_{\kappa(x)}^{\mathrm{ab}} \rightarrow$ $\pi_{1}^{\mathrm{ab}}(X)$, which for proper varieties factors through $\mathrm{SK}_{1}(X)$.

The main strategy to understand the reciprocity map $\rho^{X}$ of a smooth proper variety $X$ over a local field $K$ is to find a suitable model $\mathfrak{X}$ for $X$ over the corresponding ring of integers and relate it to the reciprocity map

$$
\rho^{Y}: \mathrm{CH}_{0}(Y) \rightarrow \pi_{1}^{\mathrm{ab}}(Y)
$$

of its reduction $Y=\mathfrak{X}_{s}$ leading to higher dimensional class field theory over finite fields $k$, which is well understood, but only for smooth and proper varieties (cf. [Lan56], [Blo81], KS83], [KS09]): For a smooth and proper variety $Y$ over a finite field $k$ the map $\rho^{Y}$ is injective with dense image. Assuming resolution of singularities, there exists a flat, proper and regular model $\mathfrak{X}$ for $X$ whose reduced special 
fibre is a simple normal crossing variety (cf. [CJS09], [Il09]), i.e. a variety whose irreducible components are smooth and intersect nicely, but which in general will not be smooth. So even if we assume that such a resolution exists, one has to deal with the reciprocity map of simple normal crossing varieties over finite fields. The cokernel and kernel of $\rho^{Y}$ then are controlled by the first and second weight homology groups associated to the dual complex $\Gamma_{Y}$ of $Y$, which only carries information about how the irreducible components of $Y$ intersect with each other.

Given such a model from above, the relation between the generic and special fibre is then given via a generalized Hasse principle for the model $\mathfrak{X}$, which was proven by U. Jannsen, S. Saito and M. Kerz (cf. [JS03], [KS10]) and which is formulated in terms of Kato homology groups $\mathrm{H}_{a}^{K}$ associated to the étale homology theory $\mathrm{H}$ and solving Kato's conjectures. More precisely, the étale homology theory over the valuation $\operatorname{ring} A$ of a local field $K$ with $\mathbb{Z} / n$-coefficients, $n$ not divisible by the residue field characteristic $p$, induces a niveau spectral sequence

$$
E_{r, q}^{1}(\mathfrak{X})=\bigoplus_{x \in \mathfrak{X}_{r}} \mathrm{H}^{r-q-2}(\kappa(x), \mathbb{Z} / n(r-1)) \Longrightarrow \mathrm{H}_{r+q}(\mathfrak{X}, \mathbb{Z} / n),
$$

for every proper scheme $\mathfrak{X}$ over $A$ and its fibres. The corresponding 5-term sequences of the model $\mathfrak{X}$, the variety $X$ and its reduction $Y$ then lie in an exact sequence. The occurring groups can be identified with modulo $n$ versions of all the appearing groups from above including Kato homology groups, which then take control over the cokernels and kernels of the reciprocity maps. These identifications use the duality theorem for étale homology theory, coming from Artin-Verdier duality together with duality theorems for Galois cohomology of the ground fields, and the lower Galois symbol isomorphisms, which come from Kummer theory and MS83, and which are part of the now proven Bloch-Kato conjecture (cf. VVoe10b], [Voe10a], [SJ06], [HW09]). Then the Hasse principle states that the Kato homology groups of the model $\mathfrak{X}$ vanish and therefore induces isomorphisms between the Kato homology groups of the variety $X$ and its reduction $Y$. Altogether, we get the commutative diagram of exact sequences

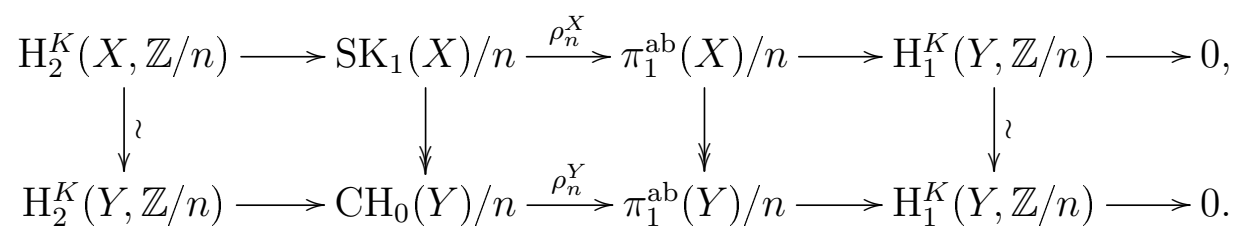

And there is also an isomorphism between $\mathrm{H}_{a}^{K}(Y, \mathbb{Z} / n)$ and the corresponding weight homology groups $\mathrm{H}_{a}\left(\Gamma_{Y}, \mathbb{Z} / n\right)$ of $\Gamma_{Y}$. So in the modulo $n$ version the first and second weight homology of the dual complex of $Y$ control the cokernel and kernel of the reciprocity map $\rho_{n}^{X}$ of $X$. 
Since the topological cokernel of the reciprocity map coker $\rho^{X}$ can be identified with $\pi_{1}^{\mathrm{ab}}(X)^{\mathrm{cd}}$, the quotient of $\pi_{1}^{\mathrm{ab}}(X)$ classifying the abelian coverings in which every closed point of $X$ is completely decomposed, and which easily is computed (cf. JJS03 Lem. 5.4) to be the first weight homology group of $\Gamma_{Y}$ in the setting from above, the whole problem will be to analyse the kernel of the reciprocity map.

The main result in this thesis concerning the kernel of the reciprocity map $\operatorname{ker} \rho^{X}$ is the following

Theorem. Let $X$ be a smooth and proper variety over a local field $K$ and let $\mathbb{L}$ be a set of prime numbers not containing the residue characteristic $p=\operatorname{char}(k)$. Then the kernel of the reciprocity map $\operatorname{ker} \rho^{X}$ is the direct sum of a finite group and a $\mathbb{L}$-divisible group.

If $\operatorname{char}(K)=0, X$ is projective over $K$ and has a model $\mathfrak{X}$ like above and which satisfies the Hasse principle for $\mathbb{Q}_{p} / \mathbb{Z}_{p}$-coefficients, then $p$ can also be included into $\mathbb{L}$. The theorem is a higher dimensional generalisation of an analogous result for curves by S. Saito (cf. [Sai85a], [Yos03]) and for surfaces by S. Saito and U. Jannsen (cf. [JS03]).

The techniques for the determination of the structure of $\operatorname{ker} \rho^{X}$ are the following. Using Gabber's refinement of de Jong's alteration theorem (cf. [Ill09], dJ96]) one can restrict to smooth, projective and geometrically irreducible $X$ with strict semi-stable reduction $Y$. Reducing to the case of a curve (cf. [Sai85a, [Yos03]), one can show that the kernel $V(X)$ of the norm map $\operatorname{SK}_{1}(X) \rightarrow K^{\times}$is a direct sum of a torsion group and a divisible group. Since $\operatorname{ker} \rho^{X}$ is a subgroup of $V(X)$ with a finite index by the structure theorem of the geometric part $\pi_{1}^{\text {geo }}(X)$ of $\pi_{1}^{\mathrm{ab}}(X)$ (cf. [Yos03]) for such $X$, one deduces that $\operatorname{ker} \rho^{X}$ also is the direct sum of a torsion group and a divisible group.

Because $\pi_{1}^{\mathrm{ab}}(X)$ and coker $\rho^{X}$ can be shown to have only a finite torsion subgroup, we get that for any set of prime numbers $\mathbb{L}$ the $\mathbb{L}$-completion of $\operatorname{ker} \rho^{X}$ coincides with the kernel of $\rho_{\mathbb{L}}^{X}$ which is the induced map from the $\mathbb{L}$-completed groups $\operatorname{SK}(X)_{\mathbb{L}} \rightarrow \pi_{1}^{\mathrm{ab}}(X)_{\mathbb{L}}$. Now one can prove inductively, reducing to the known case of a surface (cf. [JS03]) by finding a good divisor $Z \subseteq X$, that for almost all primes $\ell \in \mathbb{L}$ the $\ell$-completed group $\operatorname{ker} \rho_{\{\ell\}}^{X}$ lies in the image of $V(Z)$, which is $\ell$-divisible for such $\ell$, and therefore vanishes. By this argument we can assume that w.l.o.g. $\mathbb{L}$ is a finite set of primes. Now using the deep result that the second weight homology group $\mathrm{H}_{2}\left(\Gamma_{Y}, \mathbb{Z}\right) \otimes \mathbb{Z}_{\mathbb{L}}$, which is a finitely generated $\mathbb{Z}_{\mathbb{L}}$-module, surjects onto $\operatorname{ker} \rho_{\mathbb{L}}$, the latter group has only a finite torsion subgroup for finite sets $\mathbb{L}$. Since $\left(\operatorname{ker} \rho^{X}\right)_{\mathbb{L}}=\operatorname{ker} \rho_{\mathbb{L}}^{X}$ and $\operatorname{ker} \rho^{X}$ is torsion plus divisible, it must follow that $\operatorname{ker} \rho^{X}$ already is the direct sum of a finite group and a $\mathbb{L}$-divisible group. 
This thesis is divided into three chapters. In the first chapter we will discuss class field theory for varieties $Y$ over finite fields $k$. We will recapitulate the main definitions like Chow groups, the reciprocity map, the dual complex and quote the theorem about the injectivity of the reciprocity map for smooth and proper varieties. Using this theorem and an algebraic Seifert-van-Kampen theorem Sti06] we will be able to give a description of the kernel and cokernel of the reciprocity map for simple normal crossing varieties $Y$ in terms of weight homology groups $H_{a}\left(\Gamma_{Y}, \hat{\mathbb{Z}}\right)$ of the dual complex $\Gamma_{Y}$ in a new way and as a homology group of the complex $\pi_{1}^{\mathrm{ab}}\left(Y^{[\bullet]}\right)$ filled with descent data. This enables us to give criteria for the injectivity of the reciprocity map $\rho^{Y}$. The main results in this thesis about simple normal crossing varieties over finite fields is summarized in the following

Theorem. Let $Y=\bigcup_{i=1}^{m} Y_{i}$ be a proper simple normal crossing variety over a finite field $k$ with its irreducible components $Y_{i} \hookrightarrow Y$. Let

$$
Y^{[k]}:=\coprod_{i_{0}<i_{1}<\cdots<i_{k}} Y_{i_{0}} \times_{Y} Y_{i_{1}} \times_{Y} \ldots \times_{Y} Y_{i_{k}}
$$

be the disjoint union of the $k$-fold intersections, $k \geqslant 0$, and $\Gamma_{Y}$ be the corresponding dual complex. Consider the complex

$$
\pi_{1}^{\mathrm{ab}}\left(Y^{[\bullet]}\right): \quad \ldots \stackrel{d_{2}}{\longrightarrow} \pi_{1}^{\mathrm{ab}}\left(Y^{[1]}\right) \stackrel{d_{1}}{\longrightarrow} \pi_{1}^{\mathrm{ab}}\left(Y^{[0]}\right) \stackrel{d_{0}}{\longrightarrow} \pi_{1}^{\mathrm{ab}}(Y)
$$

with $d_{k}:=\sum_{j=0}^{k}(-1)^{j}\left(\delta_{j}^{k}\right)_{*}$

1. The cokernel and kernel of the reciprocity map

$$
\rho^{Y}: \mathrm{CH}_{0}(Y) \longrightarrow \pi_{1}^{\mathrm{ab}}(Y)
$$

then are given by

$$
\begin{aligned}
\operatorname{coker} \rho^{Y} & \cong \mathrm{H}_{-1}\left(\pi_{1}^{\mathrm{ab}}\left(Y^{[\bullet]}\right)\right) \oplus(\hat{\mathbb{Z}} / \mathbb{Z})^{\pi_{0}(Y)}, \\
\operatorname{ker} \rho^{Y} & \cong \mathrm{H}_{0}\left(\pi_{1}^{\mathrm{ab}}\left(Y^{[\bullet]}\right)\right),
\end{aligned}
$$

where the last group is finite.

2. There also is an exact sequence

$$
\mathrm{H}_{2}\left(\Gamma_{Y}, \hat{\mathbb{Z}}\right) \longrightarrow \mathrm{CH}_{0}(Y) \stackrel{\rho^{Y}}{\longrightarrow} \pi_{1}^{\mathrm{ab}}(Y) \longrightarrow(\hat{\mathbb{Z}} / \mathbb{Z})^{\pi_{0}(Y)} \oplus \mathrm{H}_{1}\left(\Gamma_{Y}, \hat{\mathbb{Z}}\right) \longrightarrow 0 .
$$

3. If, furthermore, every component of $Y^{[0]}$ and $Y^{[1]}$ is geometrically connected over $k$ and $\mathrm{CH}_{0}\left(Y^{[0]}\right)$ is torsion-free, then $\rho^{Y}$ is injective. 
The last point gives a new criterion for the injectivity of the reciprocity map for simple normal crossing varieties which does not use the second weight homology group $\mathrm{H}_{2}\left(\Gamma_{Y}, \hat{\mathbb{Z}}\right)$.

The second chapter is about homology theories following [JS03]. There we will collect all ingredients like the niveau spectral sequences, étale homology theory and the connection between the fibres of a scheme over a discrete valuation ring, we will use in the last chapter. Some homology groups can be related to each other using Kato's conjecture about the generalized Hasse principle.

In the third chapter we will do class field theory for varieties $X$ over local fields $K$. By studying the 5-term sequences of the niveau spectral sequences associated to the étale homology theory, we will be able to deduce the modulo $n$ results for the reciprocity map of varieties over local fields like in [JS03, by identifying the occurring groups with the given ones and relate them to the corresponding groups of the reduction. Therefore we will use duality theorems and some cases of the Bloch-Kato-conjecture. After an excursion over abelian groups and some quotations of some necessary theorems, we will be able to dispose ourselves from the $\mathbb{Z} / n$-coefficients. The main theorem then can be stated and proven. In the last section we will discuss, if it is probable that the kernel ker $\rho_{n}^{X}$ of the reciprocity map modulo $n$ injects into the corresponding kernel $\operatorname{ker} \rho_{n}^{Y}$ of the reduction. We will give a criterion for the failing of this by computing the orders of homology groups coming from higher terms of the niveau spectral sequence.

\section{Acknowledgement}

I heartily thank my advisor Prof. Dr. Uwe Jannsen for the opportunity to work on the interesting field of higher dimensional class field theory and his support during the creation of this thesis. I am also very thankful for the excellent working atmosphere in the whole working group in Regensburg. I also express my gratitude towards Timo Keller, Franziska Schneider, Bernd Schober and Jochen Gärtner for various correction hints. 


\section{Contents}

Introduction ............................... ii

$\begin{array}{lll}1 & \text { Class field theory over finite fields } & 1\end{array}$

1.1 The dual complex . . . . . . . . . . . . . . . . . . 1

1.2 An algebraic Seifert-van-Kampen theorem . . . . . . . . . . . . . . 4

1.3 Chow groups $\ldots \ldots \ldots \ldots \ldots$. . . . . . . . . . . . . . . . . . . . . 7

1.4 The reciprocity map over finite fields . . . . . . . . . . . . . . . . . . . . . . 10

1.5 Examples $\ldots \ldots \ldots \ldots \ldots$

2 Homology theories 25

2.1 Homology theories and their niveau spectral sequence . . . . . . . . 25

2.2 Homology theories over fields f . . . . . . . . . . . . . . . . 31

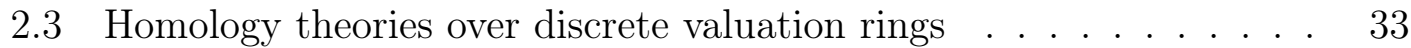

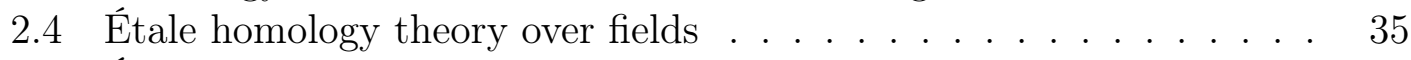

2.5 Étale homology theory over discrete valuation rings . . . . . . . . . 43

3 Class field theory over local fields $\quad 49$

3.1 The reciprocity map over local fields modulo $n$. . . . . . . . . . . . 49

3.2 Divisibility yoga and completeness meditations . . . . . . . . . . . 54

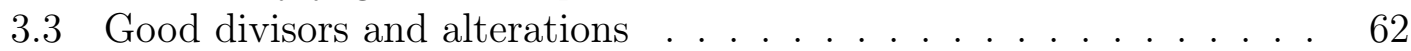

3.4 The structure of $\mathrm{SK}_{1}$ and the kernel of the reciprocity map . . . . . 64

3.5 The kernel of the reciprocity map and its reduction . . . . . . . . . 77

\begin{tabular}{lr}
\hline Bibliography & 91
\end{tabular} 


\section{Class field theory over finite fields}

The main goal of this chapter is to extend the results of class field theory of smooth proper varieties over finite fields to proper simple normal crossing varieties over finite fields. This could be done via the study of homology theories like in the next chapter (cf. JS03]), or alternatively by using a general Seifert-van-Kampen theorem. We will take the second method, for which one directly can see where the kernel and cokernel of the reciprocity map comes from. Also there are no problems with the characteristic of the field.

\subsection{The dual complex}

In this section, we will introduce the dual complex of a variety. It only contains the information of how the components of a variety meet each other. The homology groups of the dual complex play a key role in describing the kernel and cokernel of the reciprocity map in the next sections.

Definition 1.1.1 (The dual complex). Let $(I,<)$ be an totally ordered set. Let $Y=\bigcup_{i \in I} Y_{i}$ be a locally noetherian scheme with closed subschemes $Y_{i} \hookrightarrow Y$. For $k \geqslant 0$ we put

$$
Y^{[k]}:=\coprod_{i_{0}<i_{1}<\cdots<i_{k}} Y_{i_{0}, i_{1}, \ldots, i_{k}}
$$

as the disjoint union of

$$
Y_{i_{0}, i_{1}, \ldots, i_{k}}:=Y_{i_{0}} \times_{Y} Y_{i_{1}} \times_{Y} \ldots \times_{Y} Y_{i_{k}}
$$

the $k$-fold scheme-theoretic intersection of the $Y_{i}$ and $Y^{[-1]}:=Y$.

For instance we have

$$
Y^{[0]}=\coprod_{i_{0} \in I} Y_{i_{0}} \quad \text { and } \quad Y^{[1]}=\coprod_{i_{0}<i_{1} \in I} Y_{i_{0}} \cap Y_{i_{1}} .
$$

For every interger $k \geqslant 1$ there are $k+1$ morphisms

$$
\delta_{j}^{k}: Y^{[k]} \rightarrow Y^{[k-1]} \quad \text { for } j=0, \ldots, k
$$


given by the closed immersions

$$
\delta_{j}^{k}: Y_{i_{0}, \ldots, i_{k}} \hookrightarrow Y_{i_{0}, \ldots, \hat{i_{j}}, \ldots, i_{k}},
$$

where the index $i_{j} \in I$ is omitted on the right side. For $j=0, \ldots, k$ we get induced maps:

$$
\partial_{j}^{k}: \pi_{0}\left(Y^{[k]}\right) \rightarrow \pi_{0}\left(Y^{[k-1]}\right)
$$

on the connected components. Notice that we also have a canonical map $h=\delta_{0}^{0}$ : $Y^{[0]} \rightarrow Y=Y^{[-1]}$ and correspondig induced maps.

Therefore $\Gamma:=\left(\pi_{0}\left(Y^{[\bullet]}\right),\left(\partial_{j}^{\bullet}\right)_{j}\right)$ is a simplicial complex, called the dual complex to $\left(Y,\left(Y_{i}\right)_{i \in I},(I,<)\right)$.

Remark 1.1.2. $\quad$ 1. Notice that the indices are shifted by one in comparison to other papers like [JS03] or [Sat05].

2. If we only considere the vertices $\Gamma_{0}$, the edges $\Gamma_{1}$ and the faces $\Gamma_{2}$ of the simplicial complex $\Gamma$, then we have a undirected graph in the sense of graph theory.

3. If we assume that all the $Y_{i}$ are connected, then all edges between two vertices $Y_{i_{0}}$ and $Y_{i_{1}}$ are given by $\pi_{0}\left(Y_{i_{0}} \cap Y_{i_{1}}\right)$ and the faces spanned by three vertices $Y_{i_{0}}, Y_{i_{1}}$ and $Y_{i_{2}}$ are given by $\pi_{0}\left(Y_{i_{0}} \cap Y_{i_{1}} \cap Y_{i_{2}}\right)$.

Definition 1.1.3 (Simplicial homology groups). Let $\Gamma$ be simplicial complex (e.g. like the one in 1.1.1). For an abelian group $A$ we get a complex

$$
C(\Gamma, A): \ldots \stackrel{d_{3}}{\longrightarrow} A^{\left(\Gamma_{2}\right)} \stackrel{d_{2}}{\longrightarrow} A^{\left(\Gamma_{1}\right)} \stackrel{d_{1}}{\longrightarrow} A^{\left(\Gamma_{0}\right)} \stackrel{d_{0}}{\longrightarrow} A^{\left(\Gamma_{-1}\right)} \ldots
$$

with $d_{k}:=\sum_{j=0}^{k}(-1)^{j} \partial_{j}^{k}$. Therefore we get simplicial homology groups

$$
\mathrm{H}_{k}(\Gamma, A):=\operatorname{ker}\left(d_{k}\right) / \operatorname{im}\left(d_{k+1}\right) .
$$

In the setting of 1.1.1, this complex is

$$
C(\Gamma, A): \quad \ldots \stackrel{d_{3}}{\longrightarrow} A^{\left(\pi_{0}\left(Y^{[k]}\right)\right)} \stackrel{d_{2}}{\longrightarrow} A^{\left(\pi_{0}\left(Y^{[1]}\right)\right)} \stackrel{d_{1}}{\longrightarrow} A^{\left(\pi_{0}\left(Y^{[0]}\right)\right)}
$$

Notice that $A^{\left(\pi_{0}\left(Y^{[k]}\right)\right)}$ is placed in degree $k$.

Definition 1.1.4 (Maximal subtree of a complex). Let $\Gamma$ be a connected simplicial complex. A maximal subtree $T$ of $\Gamma$ is a connected and simple undirected subgraph of $\Gamma$ with the same vertices as $\Gamma$, but without any cycles, i.e.:

$$
\begin{aligned}
& T_{i}=\varnothing \quad \text { for every } i \geqslant 2, \\
& T_{i}=\Gamma_{i} \quad \text { for every } i \leqslant 0
\end{aligned}
$$

and $\# T_{1}$ is minimal with the connectivity property. (If $\# \Gamma_{0}$ is finite, then $\# T_{1}=$ $\# G_{0}-1$.) 
Lemma 1.1.5. Let $\Gamma$ be a connected simplicial complex and $T$ a maximal subtree of $\Gamma$. For every abelian group $A$ considere the vertical exact sequence of complexes:

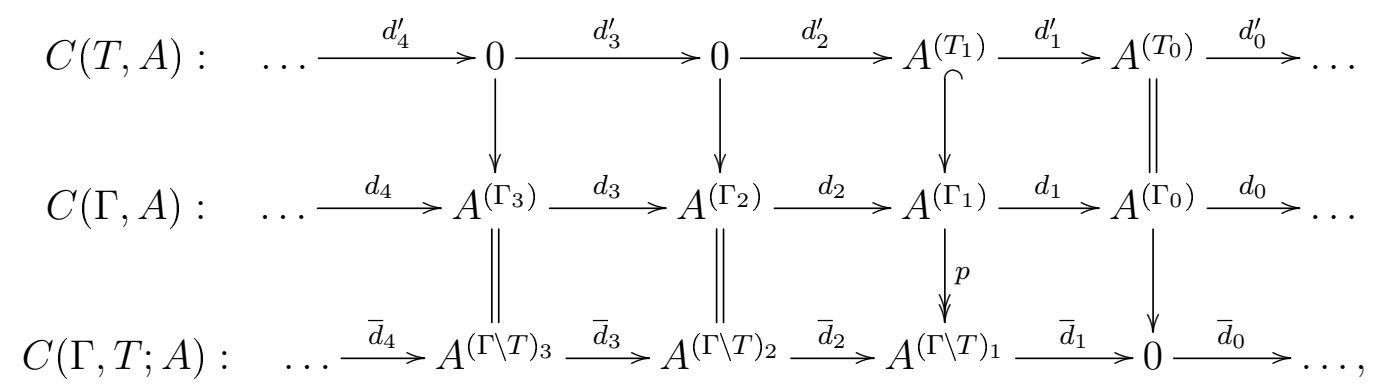

where $A^{(\Gamma \backslash T)_{i}}:=A^{\left(\Gamma_{i}\right)} / A^{\left(T_{i}\right)}$. We then have:

- $d_{1}^{\prime}$ is injective and $\mathrm{H}_{1}(T, A)=0$.

- $\operatorname{ker} d_{2}=\operatorname{ker} \bar{d}_{2}$.

- $\mathrm{H}_{i}(\Gamma, A)=\mathrm{H}_{i}(T, A)$ for $i \leqslant 0$.

- $\mathrm{H}_{i}(\Gamma, A)=\mathrm{H}_{i}(\Gamma, T ; A)$ for $i \geqslant 1$.

Proof. The first point follows from the condition on $T_{1}$ in 1.1.4. The second follows from the first with $\bar{d}_{2}=p \circ d_{2}$ from the commutative diagramm of exact sequences:

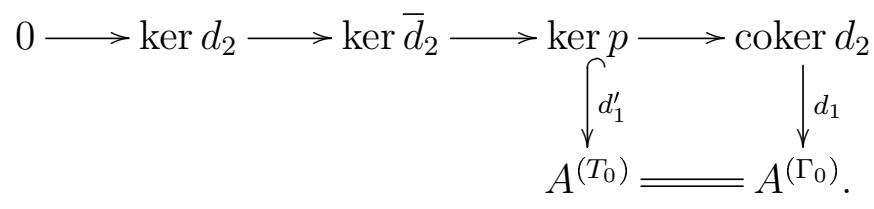

Therefore ker $p$ injects into coker $d_{2}$ and the sequence splits off an isomorphism on the left. And the rest then follows from the long exact sequence.

Lemma 1.1.6 (The connected components). Let $Y=\bigcup_{v \in I} Y_{v}$ be a noetherian scheme which is covered by a finite number of closed subschemes $Y_{v} \subseteq Y$ and let $\Gamma$ be the dual complex to $\left(Y,\left(Y_{i}\right)_{i \in I},(I,<)\right)$. Then we have:

1. If $Y$ is connected, then for every pair of indices $\left(v, v^{\prime}\right)$ there is a finite sequence of indices $v=v_{0}, \ldots, v_{r}=v^{\prime}$ such that

$$
Y_{v_{j}} \cap Y_{v_{j+1}} \neq \varnothing
$$

for every $j=0, \ldots, r-1$. In particular, the dual complex $\Gamma$ is also connected.

2. If every $Y_{v}$ is connected, then $Y$ is connected if and only if the above condition holds. 
3. If every $Y_{v}$ is connected (and $Y$ may not be connected), then there is a canonical isomorphism

$$
A^{\pi_{0}(\Gamma)} \cong \mathrm{H}_{0}(\Gamma, A) \stackrel{\sum}{\cong} A^{\pi_{0}(Y)}
$$

for every abelian group $A$.

Proof. If the $Y_{v}$ are exactly the irreducible components of $Y$ the second point can be found in [Liu02] §2.4 Ex. 4.4. The statements then can be deduced from this, since every closed subset must intersect some irreducible component of $Y$ and every irreducible component must lie in at least one of the $Y_{v}$.

\subsection{An algebraic Seifert-van-Kampen theorem}

Here we will cite an algebraic version of the Seifert-van-Kampen theorem. It allows us to compute the fundamental group of a variety out of the fundamental groups of its irreducible components and their multiple intersections. In particular, we can describe the abelianized fundamental group of that variety. Unfortunately, there is a lack of information, which can be controlled by the homology groups of the dual complex.

Theorem 1.2.1 (Algebraic Seifert-van-Kampen Theorem). Let $(I,<)$ be a finite and totally ordered set and $Y=\bigcup_{v \in I} Y_{v}$ be a locally noetherian and connected scheme with closed and connected subschemes $Y_{v} \hookrightarrow Y$. Let $h: Y^{[0]}=\coprod_{v \in I} Y_{v} \rightarrow$ $Y$ be the canonical map.

Let $\bar{s}$ be a geometric point of $Y$ and for every $k=0,1,2$ and every $t \in \pi_{0}\left(Y^{[k]}\right)$ let $\bar{s}(t)$ be a geometric point of $t$. Let $\Gamma$ be the dual complex to $\left(Y,\left(Y_{i}\right)_{i \in I},(I,<)\right)$. Fix a maximal subtree $T$ of $\Gamma$ and for every boundary map $\partial: t \rightarrow t^{\prime}$ in $\Gamma_{\leqslant 2}$ let $\gamma_{t, t^{\prime}}: \bar{s}\left(t^{\prime}\right) \leadsto \Gamma(\partial) \bar{s}(t)$ be a fixed path in the sense of algebraic paths between base points, i.e. a fixed isomorphism between the corresponding fibre functors. Then canonically with respect to all these choices we have an isomorphism

$$
\pi_{1}(Y, \bar{s}) \cong\left(\left(\underset{v \in I}{*} \pi_{1}\left(Y_{v}, \bar{s}(v)\right)\right) * \hat{\pi}_{1}(\Gamma, T)\right) / H,
$$

where $H$ is the closed normal subgroup generated by the cocycle and edge relations:

$$
\begin{aligned}
\operatorname{edg}\left(e, g_{e}\right) & :=\vec{e} \cdot \pi_{1}\left(\delta_{0}^{1}\right)\left(g_{e}\right) \cdot(\vec{e})^{-1} \cdot \pi_{1}\left(\delta_{1}^{1}\right)\left(g_{e}\right)^{-1} \\
\operatorname{coc}(f) & :=\left(\overrightarrow{\partial_{2}^{2} f}\right) \cdot \alpha_{102}^{(f)}\left(\alpha_{120}^{(f)}\right)^{-1} \cdot\left(\overrightarrow{\partial_{0}^{2} f}\right) \cdot \alpha_{210}^{(f)}\left(\alpha_{201}^{(f)}\right)^{-1} \cdot\left(\overrightarrow{\partial_{1}^{2} f}\right)^{-1} \cdot \alpha_{021}^{(f)}\left(\alpha_{012}^{(f)}\right)^{-1},
\end{aligned}
$$

for all parameter values $e \in \Gamma_{1}, g_{e} \in \pi_{1}(e, \bar{s}(e))$ and $f \in \Gamma_{2} . \vec{e}$ is defined to be the corresponing topological generators of the pro-finite group

$$
\left.\hat{\pi}_{1}(\Gamma, T):=\underset{e \in \Gamma_{1}}{*} \hat{\mathbb{Z}}\right) /\left(\overrightarrow{e^{\prime}} \mid e^{\prime} \in T_{1}\right) \cong \underset{e \in \Gamma_{1} \backslash T_{1}}{*} \hat{\mathbb{Z}}
$$


The map $\pi_{1}\left(\delta_{i}^{1}\right)$ uses the fixed path $\gamma_{\delta_{i}^{1}(e), e}$. And finally $\alpha_{i j k}^{(f)}$ is defined using the $i$-th vertex $v=v_{i}(f) \in \Gamma_{0}$ of $f$ and the edge $e=e_{i j}(f) \in \Gamma_{1}$ with vertices $\left\{v_{i}(f), v_{j}(f)\right\}=$ $\left\{\partial_{0}^{1}(e), \partial_{1}^{1}(e)\right\}$ as

$$
\alpha_{i j k}^{(f)}:=\gamma_{v_{i}(f), e_{i j}(f)} \circ \gamma_{e_{i j}(f), f} \circ\left(\gamma_{v_{i}(f), f}\right)^{-1} \in \pi_{1}\left(Y_{v_{i}}, \bar{s}\left(v_{i}\right)\right) .
$$

Proof. This is a special case of [Sti06] Corollary 5.4 noting that closed immersions are monomorphisms ([Sti06] Definition 4.2) and using [Sti06] Theorem 5.2(1) to see that $h$ - a proper, surjective morphism of finite presentation - is a universal effective descent morphism for finite étale covers ([Sti06] Definition 5.1).

Remark 1.2.2. If we replace the covering of closed subschemes $\left(Y_{i}\right)_{i \in I}$ by a covering of open subschemes, theorem 1.2.1 also holds. $h$ is then faithfully flat (i.e. flat and surjective) and of finite presentation and therefore a universal effective descent morphism for finite étale covers by [Sti06] Theorem 5.2(2).

Corollary 1.2.3 (The abelianized fundamental group). Let the notations be as in 1.2.1. The abelianized fundamental group of $Y$ is then given by

$$
\pi_{1}^{\mathrm{ab}}(Y)=\left(\pi_{1}^{\mathrm{ab}}\left(Y^{[0]}\right) \oplus \hat{\pi}_{1}^{\mathrm{ab}}(\Gamma, T)\right) / \bar{H},
$$

where we put:

$$
\begin{aligned}
\pi_{1}^{\mathrm{ab}}\left(Y^{[k]}\right) & :=\bigoplus_{Z \in \pi_{0}\left(Y^{[k]}\right)} \pi_{1}^{\mathrm{ab}}(Z), \\
\hat{\pi}_{1}^{\mathrm{ab}}(\Gamma, T) & :=\left(\bigoplus_{e \in \Gamma_{1}} \hat{\mathbb{Z}}\right) /\left(\overrightarrow{e^{\prime}} \mid e^{\prime} \in T_{1}\right) \\
& \cong \bigoplus_{e \in \Gamma_{1} \backslash T_{1}} \hat{\mathbb{Z}} .
\end{aligned}
$$

And $\bar{H}$ is topologically generated by the relations

$$
d_{1}(g) \quad \text { and } \quad \overline{\operatorname{coc}}(f)=\beta(f)+\bar{d}_{2}(f),
$$

where

$$
\begin{aligned}
d_{1}: \pi_{1}^{\mathrm{ab}}\left(Y^{[1]}\right) & \rightarrow \pi_{1}^{\mathrm{ab}}\left(Y^{[0]}\right), \\
g & \mapsto \pi_{1}^{\mathrm{ab}}\left(\delta_{0}^{1}\right)(g)-\pi_{1}^{\mathrm{ab}}\left(\delta_{1}^{1}\right)(g), \\
\bar{d}_{2}: \bigoplus_{f \in \Gamma_{2}} \hat{\mathbb{Z}} & \rightarrow \hat{\pi}_{1}^{\mathrm{ab}}(\Gamma, T), \\
\sum_{f} n_{f} \cdot f & \mapsto \sum_{f} n_{f} \cdot\left(\overrightarrow{\partial_{0}^{2} f}-\overrightarrow{\partial_{1}^{2} f}+\overrightarrow{\partial_{2}^{2} f}\right), \\
\beta: \quad \bigoplus_{f \in \Gamma_{2}} \hat{\mathbb{Z}} & \rightarrow \pi_{1}^{\mathrm{ab}}\left(Y^{[0]}\right) \\
\sum_{f} n_{f} \cdot f & \mapsto \sum_{f} n_{f} \cdot\left(\bar{\alpha}_{102}^{(f)}-\bar{\alpha}_{120}^{(f)}+\bar{\alpha}_{210}^{(f)}-\bar{\alpha}_{201}^{(f)}+\bar{\alpha}_{021}^{(f)}-\bar{\alpha}_{012}^{(f)}\right), \\
\overline{\mathrm{coc}}: \bigoplus_{f \in \Gamma_{2}} \hat{\mathbb{Z}} & \rightarrow \pi_{1}^{\mathrm{ab}}\left(Y^{[0]}\right) \oplus \hat{\pi}_{1}^{\mathrm{ab}}(\Gamma, T), \\
\sum_{f} n_{f} \cdot f & \mapsto \sum_{f} n_{f} \cdot\left(\beta(f)+\bar{d}_{2}(f)\right),
\end{aligned}
$$


writing all the abelian groups additively. Furthermore, for an abelian group A consider the complex

$$
\pi_{1}^{\mathrm{ab}}\left(Y^{[\bullet]}\right) \otimes_{\mathbb{Z}} A: \quad \pi_{1}^{\mathrm{ab}}\left(Y^{[1]}\right) \otimes_{\mathbb{Z}} A \stackrel{d_{1}}{\longrightarrow} \pi_{1}^{\mathrm{ab}}\left(Y^{[0]}\right) \otimes_{\mathbb{Z}} A \stackrel{d_{0}}{\longrightarrow} \pi_{1}^{\mathrm{ab}}(Y) \otimes_{\mathbb{Z}} A .
$$

with homology groups $\mathrm{H}_{i}\left(\pi_{1}^{\mathrm{ab}}\left(Y^{[\bullet]}\right), A\right)$. We then have an isomorphism

$$
\mathrm{H}_{1}(\Gamma, A) \cong \mathrm{H}_{-1}\left(\pi_{1}^{\mathrm{ab}}\left(Y^{[\bullet]}\right), A\right):=\operatorname{coker}\left(d_{0}\right),
$$

and a surjection

$$
\mathrm{H}_{2}(\Gamma, A) \stackrel{\beta_{l}}{\rightarrow} \mathrm{H}_{0}\left(\pi_{1}^{\mathrm{ab}}\left(Y^{[\bullet]}\right), A\right):=\operatorname{ker}\left(d_{0}\right) / \operatorname{im}\left(d_{1}\right) .
$$

Proof. The first statement immediately follows from 1.2.1 by abelianization. For clarity in the description of the homology groups we suppress the terms $\otimes_{\mathbb{Z}} A$ in every line. Now $d_{0}$ is the canonical map

$$
d_{0}: \pi_{1}^{\mathrm{ab}}\left(Y^{[0]}\right) \rightarrow \pi_{1}^{\mathrm{ab}}(Y)=\left(\left(\pi_{1}^{\mathrm{ab}}\left(Y^{[0]}\right) \oplus \hat{\pi}_{1}^{\mathrm{ab}}(\Gamma, T)\right) / \bar{H} .\right.
$$

So we get

$$
\begin{aligned}
\mathrm{H}_{-1}\left(\pi_{1}^{\mathrm{ab}}\left(Y^{[\bullet]}\right), A\right) & =\operatorname{coker}\left(d_{0}\right) \\
& =\left(\pi_{1}^{\mathrm{ab}}\left(Y^{[0]}\right) \oplus \hat{\pi}_{1}^{\mathrm{ab}}(\Gamma, T)\right) /\left(\pi_{1}^{\mathrm{ab}}\left(Y^{[0]}\right)+\bar{H}\right) \\
& =\left(\pi_{1}^{\mathrm{ab}}\left(Y^{[0]}\right) \oplus \hat{\pi}_{1}^{\mathrm{ab}}(\Gamma, T)\right) /\left(\pi_{1}^{\mathrm{ab}}\left(Y^{[0]}\right) \oplus \operatorname{im}\left(\bar{d}_{2}\right)\right) \\
& \cong\left(\hat{\pi}_{1}^{\mathrm{ab}}(\Gamma, T)\right) / \mathrm{im}\left(\bar{d}_{2}\right) \\
& =\mathrm{H}_{1}(\Gamma, T ; A) \\
& \cong \mathrm{H}_{1}(\Gamma, A) .
\end{aligned}
$$

The last isomorphism holds because $T$ is a maximal subtree of $\Gamma$ with 1.1 .5 And we have

$$
\begin{aligned}
\mathrm{H}_{0}\left(\pi_{1}^{\mathrm{ab}}\left(Y^{[\bullet]}\right), A\right) & =\operatorname{ker}\left(d_{0}\right) / \operatorname{im}\left(d_{1}\right) \\
& =\left(\pi_{1}^{\mathrm{ab}}\left(Y^{[0]}\right) \cap \bar{H}\right) / \operatorname{im}\left(d_{1}\right) .
\end{aligned}
$$

Now consider the exact sequence

$$
0 \rightarrow\left(\pi_{1}^{\mathrm{ab}}\left(Y^{[0]}\right) \cap \bar{H}\right) / \operatorname{im}\left(d_{1}\right) \rightarrow \bar{H} / \operatorname{im}\left(d_{1}\right) \stackrel{\omega}{\rightarrow}\left(\pi_{1}^{\mathrm{ab}}\left(Y^{[0]}\right)+\bar{H}\right) / \pi_{1}^{\mathrm{ab}}\left(Y^{[0]}\right) \rightarrow 0
$$

Because $\bar{H}=\left\langle\operatorname{im}\left(d_{1}\right), \operatorname{im}(\overline{\mathrm{COC}})\right\rangle$ we have a surjection

$$
\overline{\mathrm{coc}}: \bigoplus_{f \in \Gamma_{2}} \hat{\mathbb{Z}} \rightarrow \bar{H} / \operatorname{im}\left(d_{1}\right) .
$$


And $\quad \bar{d}_{2}: \bigoplus_{f \in \Gamma_{2}} \hat{\mathbb{Z}} \rightarrow \hat{\pi}_{1}^{\mathrm{ab}}(\Gamma, T)$ factors through $\omega:$

$$
\bar{d}_{2}: \bigoplus_{f \in \Gamma_{2}} \hat{\mathbb{Z}} \stackrel{\overline{\mathrm{coc}}}{\rightarrow} \bar{H} / \operatorname{im}\left(d_{1}\right) \stackrel{\omega}{\rightarrow}\left(\pi_{1}^{\mathrm{ab}}\left(Y^{[0]}\right)+\bar{H}\right) / \pi_{1}^{\mathrm{ab}}\left(Y^{[0]}\right) \stackrel{\mathrm{pr}_{2}}{\rightarrow} \hat{\pi}_{1}^{\mathrm{ab}}(\Gamma, T)
$$

Therefore the restriction of $\overline{\operatorname{coc}}$ to $\operatorname{ker}\left(\bar{d}_{2}\right)$, which by definition coincides with the restriction of $\beta$ to $\operatorname{ker}\left(\bar{d}_{2}\right)$, induces a well-defined and surjective map

$$
\beta_{\mid}=\overline{\operatorname{coc}}_{\mid}: \operatorname{ker}\left(\bar{d}_{2}\right) \rightarrow\left(\pi_{1}^{\mathrm{ab}}\left(Y^{[0]}\right) \cap \bar{H}\right) / \operatorname{im}\left(d_{1}\right) .
$$

Because $T$ is a maximal subtree of $\Gamma$, we have $\operatorname{ker} d_{2}=\operatorname{ker} \bar{d}_{2}$ by 1.1.5. And

$$
\begin{aligned}
\beta: \bigoplus_{f \in \Gamma_{2}} \hat{\mathbb{Z}} & \rightarrow \pi_{1}^{\mathrm{ab}}\left(Y^{[0]}\right), \\
\sum_{f} n_{f} \cdot f & \mapsto \sum_{f} n_{f} \cdot\left(\bar{\alpha}_{102}^{(f)}-\bar{\alpha}_{120}^{(f)}+\bar{\alpha}_{210}^{(f)}-\bar{\alpha}_{201}^{(f)}+\bar{\alpha}_{021}^{(f)}-\bar{\alpha}_{012}^{(f)}\right),
\end{aligned}
$$

vanishes on the image of

$$
d_{3}: \bigoplus_{Z \in \Gamma_{3}} \hat{\mathbb{Z}} \rightarrow \bigoplus_{f \in \Gamma_{2}} \hat{\mathbb{Z}}
$$

by defniniton of the $\bar{\alpha}_{i j k}^{(f)}$ and alternating signs. Therefore we also get a surjection

$$
\mathrm{H}_{2}(\Gamma, A)=\operatorname{ker}\left(d_{2}\right) / \operatorname{im}\left(d_{3}\right) \stackrel{\beta_{1}}{\rightarrow} \mathrm{H}_{0}\left(\pi_{1}^{\mathrm{ab}}\left(Y^{[\bullet]}\right), A\right)
$$

which finishes the proof.

\subsection{Chow groups}

Here we will recapitulate the definition and handling of the Chow groups of a variety. The aim of class field theory over finite fields is to compute the abelianized fundamental group out of the Chow group of zero-cycles of a variety over a finite field via the reciprocity map, which will be done in the next section.

Definition 1.3.1 (Chow groups). Let $X$ be a noetherian scheme. For every integer $j \geqslant$ we put

$$
X_{j}:=\{x \in X \mid \operatorname{dim}(\overline{\{x\}})=j\}
$$

as the set of $j$-dimensional points of $X$. Furthermore, we define the Chow groups to be

$$
\mathrm{CH}_{j}(X):=\operatorname{coker}\left(\bigoplus_{y \in X_{j+1}} \kappa(y)^{\times} \longrightarrow \bigoplus_{x \in X_{j}} \mathbb{Z}\right) \text {. }
$$


Note that the maps are given by

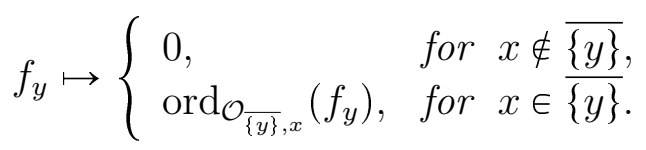

Therefore they are well-defined homomorphisms, since $\operatorname{dim} \mathcal{O}_{\overline{\{y\}}, x}=1$ in the last case.

Lemma 1.3.2 (Mayer-Vietoris sequence). Let $X$ be a scheme of finite type over a field $k$. If $X_{1}, X_{2} \hookrightarrow X$ are closed subschemes with $X=X_{1} \cup X_{2}$, then for every integer $l \geqslant 0$ there is an exact sequence:

$$
\mathrm{CH}_{l}\left(X_{1} \cap X_{2}\right) \stackrel{(-,+)}{\longrightarrow} \mathrm{CH}_{l}\left(X_{1}\right) \oplus \mathrm{CH}_{l}\left(X_{2}\right) \stackrel{i_{1 *}+i_{2 *}}{\longrightarrow} \mathrm{CH}_{l}(X) \longrightarrow 0
$$

Proof. See [Ful98] Example 1.3.1 and 1.8.1.

Lemma 1.3.3. Let $X=\bigcup_{s=1}^{r} X_{s}$ be a scheme of finite type over a field $k$ with closed subschemes $i_{s}: X_{s} \hookrightarrow X$. Then for every $l \geqslant 0$ there is an exact sequence

$$
\bigoplus_{1 \leqslant t<j \leqslant r} \mathrm{CH}_{l}\left(X_{t} \cap X_{j}\right) \stackrel{(-,+)}{\longrightarrow} \bigoplus_{s=1}^{r} \mathrm{CH}_{l}\left(X_{s}\right) \stackrel{\sum_{s} i_{s *}}{\longrightarrow} \mathrm{CH}_{l}(X) \longrightarrow 0 .
$$

The signs of the left map for $t<j$ are given by

$$
\mathrm{CH}_{l}\left(X_{t} \cap X_{j}\right) \stackrel{(-,+)}{\longrightarrow} \mathrm{CH}_{l}\left(X_{t}\right) \oplus \mathrm{CH}_{l}\left(X_{j}\right),
$$

i.e. the sequence

$$
\mathrm{CH}_{l}\left(X^{[1]}\right) \stackrel{d_{1}}{\longrightarrow} \mathrm{CH}_{l}\left(X^{[0]}\right) \stackrel{d_{0}}{\longrightarrow} \mathrm{CH}_{l}(X) \longrightarrow 0
$$

is exact with the notations of 1.1.1.

Proof. Consider

$$
X=\bigcup_{s=1}^{r} X_{s}=\left(\bigcup_{s=1}^{r-2} X_{s}\right) \cup\left(X_{r-1} \cup X_{r}\right)
$$

and

$$
X_{t} \cap\left(X_{r-1} \cup X_{r}\right)=\left(X_{t} \cap X_{r-1}\right) \cup\left(X_{t} \cap X_{r}\right) .
$$

For brevity we will write

$$
\mathrm{H}(Y):=\mathrm{CH}_{l}(Y) \quad \text { and } \quad X_{t, s}:=X_{t} \cap X_{s}
$$

and

$$
\mathrm{H}\left(X^{\prime}\right):=\bigoplus_{s=1}^{r-2} \mathrm{H}\left(X_{s}\right) \quad \text { and } \quad \mathrm{H}\left(X^{\prime \prime}\right):=\bigoplus_{t<j \leqslant r-2} \mathrm{H}\left(X_{t} \cap X_{j}\right)
$$


By induction on $r$ and successive applications of Mayer-Vietoris sequences 1.3.2. we get a commutative diagramm of exact sequences:

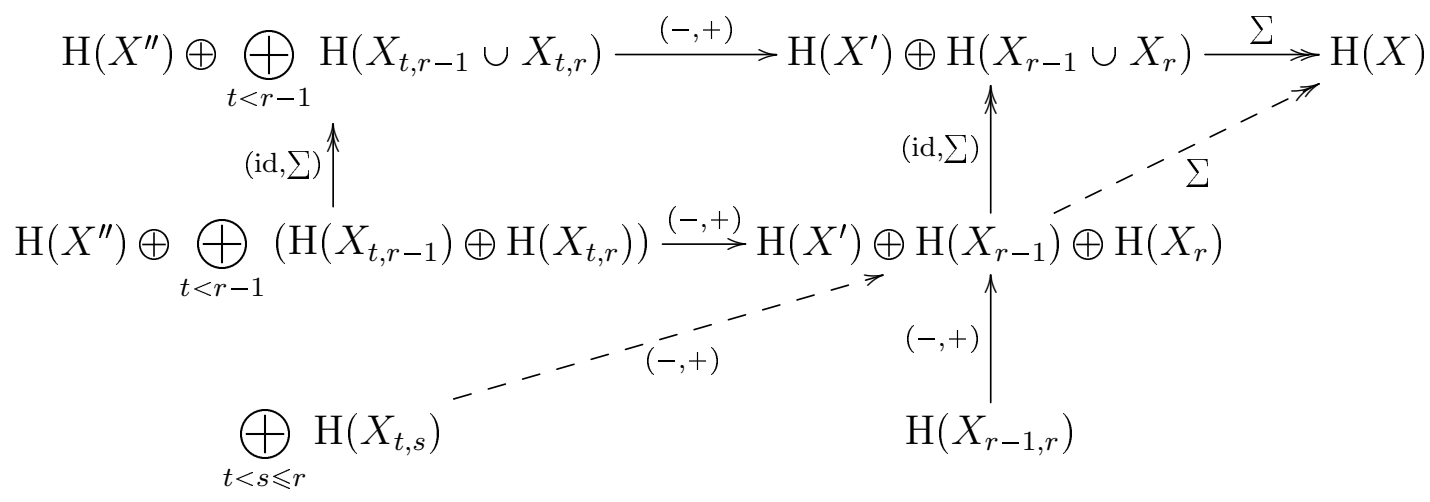

Therefore the dotted sequence is exact.

Lemma 1.3.4 (Localisation-/excision-sequence). Let $X$ be scheme of finite type over a field $k$ and $Z$ a closed subscheme and $U=X \backslash Z$ the complement. Let $i: Z \hookrightarrow X$ and $j: U \hookrightarrow X$ be the inclusion maps. Then there is an exact sequence

$$
\mathrm{CH}_{l}(Z) \stackrel{i_{*}}{\longrightarrow} \mathrm{CH}_{l}(X) \stackrel{j^{*}}{\longrightarrow} \mathrm{CH}_{l}(U) \longrightarrow 0
$$

for every integer $l \geqslant 0$.

Proof. See [Ful98] Proposition 1.8.

Definition 1.3.5. Let $X$ be a proper $k$-scheme. Then there is a well-defined degreemap:

$$
\begin{array}{ccc}
\operatorname{deg}: & \mathrm{CH}_{0}(X) \\
\sum_{P} n_{P} \cdot[P] & \mapsto & \mathbb{Z}, \\
& \sum_{P} n_{P} \cdot[\kappa(P): k],
\end{array}
$$

given by the push-forward-map of the structure morphism $p: X \rightarrow \operatorname{Spec}(k)$.

Theorem 1.3.6 (Bloch; Kato, Saito). Let $X$ be a scheme of finite type over $\mathbb{Z}$. Then $\mathrm{CH}_{0}(X)$ is a finitely generated abelian group.

If $X$ is separated and connected, then there are two possibilities:

(i) If $X$ is nonempty, proper and some prime number $p$ is nilpotent on $X$, then $\mathrm{CH}_{0}(X) \cong \mathbb{Z} \oplus T$, where $T$ is a finite group.

(ii) If $X$ does not satisfy (i) then $\mathrm{CH}_{0}(X)$ is finite.

Proof. See [Blo81] Theorem 4.2 and [KS86] Theorem 6.1 or [Ras95] for a summary. 


\subsection{The reciprocity map over finite fields}

In this section we will state the main results of class field theory over finite fields. Firstly, we recapitulate the definition of the reciprocity map of proper varieties over finite fields. Secondly, we cite the deep theorems concerning the injectivity of the reciprocity map for smooth and proper varieties over finite fields. And in the end of this section we will be able to give a description of the kernel and cokernel of the reciprocity map of proper simple normal crossing varieties over finite fields in terms of the homology groups of their dual complex using the algebraic Seifert-vanKampen-theorem. We also invent a criterion for the injectivity of the reciprocity map which does not relay on the homology of the dual complex.

Definition 1.4.1 (The reciprocity map). Let $Y$ be a scheme of finite type over $\mathbb{Z}$ and $\pi_{1}^{\mathrm{ab}}(Y)=\bigoplus_{Y^{\prime} \in \pi_{0}(Y)} \pi_{1}^{\mathrm{ab}}\left(Y^{\prime}\right)$ the abelianized étale fundamental group of $Y$. Let $y \in Y$ be a closed point. Then the residue field $\kappa(y)$ is a finite field. Therefore we can considere the image of the Frobenius automorphism $\varphi_{y} \in G_{\kappa(y)}=\pi_{1}^{\mathrm{ab}}(\operatorname{Spec}(\kappa(y))$ in $\pi_{1}^{\mathrm{ab}}(Y)$ via the push-forward of the natural map

$$
i_{y}: \operatorname{Spec}(\kappa(y)) \rightarrow Y .
$$

This mapping extends linearly to the group of zero-cycles of $Y$ :

$$
\begin{aligned}
\rho^{\prime}: Z_{0}(Y)=\bigoplus_{y \in Y_{(0)}} \mathbb{Z} \cdot y & \rightarrow \quad \pi_{1}^{\mathrm{ab}}(Y), \\
\sum_{y} n_{y} \cdot y & \mapsto \quad \sum_{y} n_{y} \cdot\left(i_{y}\right)_{*}\left(\varphi_{y}\right) .
\end{aligned}
$$

$\rho^{\prime}$ is called the reciprocity map of $Y$.

Theorem 1.4.2 (Lang). Let $Y$ be a normal integral separated scheme of finite type over $\mathbb{Z}$. The Frobenius elements then generate a dense subgroup in the toplogical group $\pi_{1}^{\mathrm{ab}}(Y)$, i.e. the reciprocity map of zero-cycles

$$
\rho^{\prime}: Z_{0}(Y) \rightarrow \pi_{1}^{\mathrm{ab}}(Y)
$$

has dense image.

Proof. The proof uses zeta-functions. See [Sza09] Theorem 5.8.16 and [Mil80] Section VI.12.

Theorem 1.4.3. Let $Y$ be a proper scheme over a finite field $k$. Then we have the following statements:

1. The reciprocity map $\rho^{\prime}$ factors through rational equivalence to give a map from the Chow group of zero-cycles

$$
\rho: \mathrm{CH}_{0}(Y) \rightarrow \pi_{1}^{\mathrm{ab}}(Y),
$$

which is also called reciprocity map of $Y$. 
2. If $Y$ is normal, then $\rho$ has dense image.

3. If $Y$ is connected and smooth over $k$, then the cokernel of $\rho$ is isomorphic to $\hat{\mathbb{Z}} / \mathbb{Z}$ (given by the degree maps).

4. If $Y$ is connected, smooth and projective over $k$, then $\rho$ is injective.

Proof. See 1.4.2, [Blo81], Lan56], [KS83] and [Ras95] §5 for a summary. Note for 3 . that the assumption of geometrically connectedness can be weakend to connectedness by the Stein factorisation of $Y \rightarrow \operatorname{Spec}(k)$ and 1.4.6, i.e. we can assume the degree-maps to be surjective.

Remark 1.4.4. The projectivity assumption in the last point of 1.4 .3 can be weakend to properness. One way to do so, is using Wiesend's class field theory (cf. [Wie06], [Wie07], [KS09], resp.) with the further assumption of a fibration over a curve. Another proof was presented on the spring school in Mainz 2011 on "Higher dimensional class field theory" by Tamás Szamuely, which we want to sketch:

Theorem 1.4.5. Let $Y$ be a proper and smooth scheme over a finite field $k$. Then the reciprocity map

$$
\rho: \mathrm{CH}_{0}(Y) \rightarrow \pi_{1}^{\mathrm{ab}}(Y),
$$

is injective and the cokernel of $\rho$ is isomorphic to $(\hat{\mathbb{Z}} / \mathbb{Z})^{\pi_{0}(Y)}$, which is a uniquely divisible group. Moreover, we have a commutative diagram of exact sequences

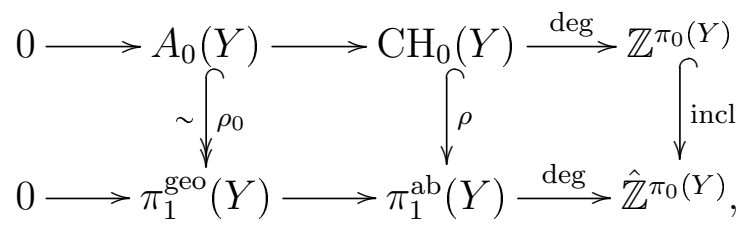

where the restriction of the reciprocity map $\rho_{0}$ induces an isomorphism of finite groups between the kernels of the degree maps $A_{0}(Y)$ and $\pi_{1}^{\mathrm{geo}}(Y)$.

Proof presented by T. Szamuely. We only have to show that $\rho$ is injective for a connected such scheme. Let $\mathbb{Z}(i):=Z^{i}\left({ }_{-}, \bullet\right)[-2 i]$ be the motivic complex on the Zariski site of $Y$ defined in [Blo86]:

$$
Z^{i}(U, q):=\left\{W \in Z^{i}\left(U \times \Delta^{q}\right) \text { meeting all faces properly }\right\},
$$

where

$$
\Delta^{q}:=\operatorname{Spec}\left(k\left[t_{0}, \ldots, t_{q}\right] /\left(\sum t_{j}-1\right)\right) .
$$

Let $\pi: Y_{\text {ét }} \rightarrow Y_{\text {Zar }}$ be the canonical map between sites. For any prime $\ell$ we get isomorphism by [SV00] and GL01:

$$
\pi^{*} \mathbb{Z}(i) \otimes \mathbb{Z} / \ell^{n} \stackrel{\sim}{\rightarrow} \mathbb{Z} / \ell^{n}(i)
$$


and

$$
\mathbb{Z}(i) \otimes \mathbb{Z} / \ell^{n} \stackrel{\sim}{\rightarrow} \tau_{\leqslant i} R \pi_{*} \mathbb{Z} / \ell^{n}(i)
$$

with a sophisticated truncation $\tau_{\leqslant i}$ and the étale sheaf

$$
\mathbb{Z} / \ell^{n}(i):= \begin{cases}\mu_{\ell^{n}}^{\otimes i} & \text { for } \quad \ell \neq \operatorname{char}(k), \\ W_{n} \Omega_{Y, \log }^{i}[-i] & \text { for } \quad \ell=\operatorname{char}(k),\end{cases}
$$

where the last one is the logarithmic part of de Rham-Witt sheaves, cf. [Ill79]. The exact triangle given by multiplication with $\ell^{n}$ :

$$
\mathbb{Z}(i) \stackrel{\ell^{n}}{\longrightarrow} \mathbb{Z}(i) \longrightarrow \mathbb{Z}(i) \otimes \mathbb{Z} / \ell^{n} \stackrel{\delta}{\longrightarrow} \mathbb{Z}(i)[1]
$$

induces the surjection

$$
\mathrm{H}_{\mathrm{Zar}}^{2 i-1}\left(Y, \mathbb{Z}(i) \otimes \mathbb{Z} / \ell^{n}\right) \rightarrow \ell^{n} \mathrm{H}_{\mathrm{Zar}}^{2 i}(Y, \mathbb{Z}(i)) \cong \ell^{n} \mathrm{CH}^{i}(Y)
$$

and the commutative diagram

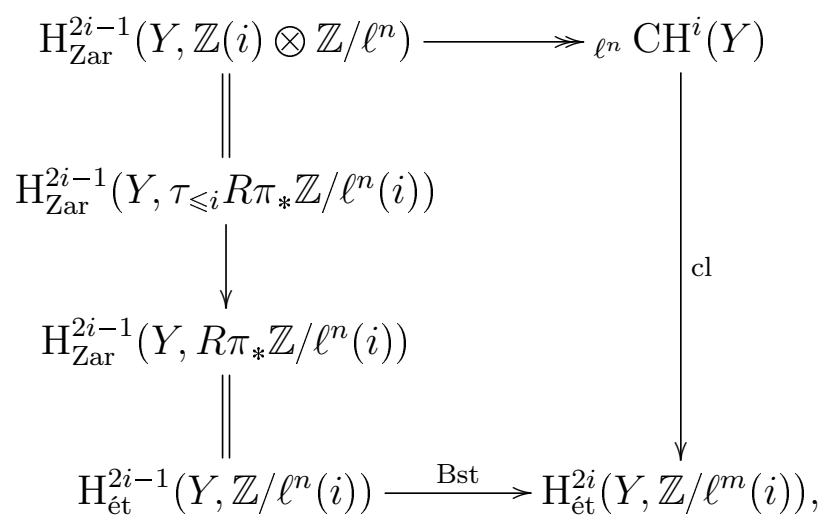

where the vertical map on the right is the cycle class map and the bottom map is the Bockstein map induced by the exact sequence

$$
0 \rightarrow \mathbb{Z} / \ell^{m} \rightarrow \mathbb{Z} / \ell^{n+m} \rightarrow \mathbb{Z} / \ell^{n} \rightarrow 0
$$

Putting $i=d=\operatorname{dim} Y$ and taking the direct limit over all $n$ on the left and the inverse limit over all $m$ on the right we get the commutative diagram

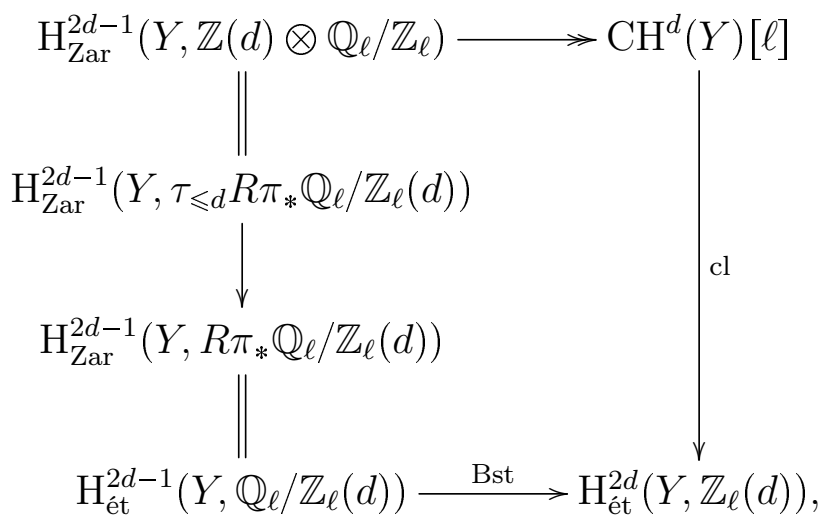


The lower map is injective because it sits in the exact sequence

$$
\mathrm{H}_{\text {ét }}^{2 d-1}\left(Y, \mathbb{Q}_{\ell}(d)\right) \rightarrow \mathrm{H}_{\text {ét }}^{2 d-1}\left(Y, \mathbb{Q}_{\ell} / \mathbb{Z}_{\ell}(d)\right) \rightarrow \mathrm{H}_{\text {ét }}^{2 d}\left(Y, \mathbb{Z}_{\ell}(d)\right),
$$

where the first group vanishes by weight arguments.

We now study the kernel of the left vertical map. By the affine Lefschetz theorem we have that $\mathrm{H}_{\mathrm{Zar}}^{a}\left(U, R \pi_{*} \mathbb{Q}_{\ell} / \mathbb{Z}_{\ell}(d)\right)=\mathrm{H}_{\text {êt }}^{a}\left(U, \mathbb{Q}_{\ell} / \mathbb{Z}_{\ell}(d)\right)$ vanishes for all $U \subseteq Y$ affine together with $a>d+1$. Therefore

$$
R^{a} \pi_{*} \mathbb{Q}_{\ell} / \mathbb{Z}_{\ell}(d)=0 \quad \text { for } \quad a>d+1
$$

So, if $d>2$ then $a:=2 d-1>d+1$ and the line above holds.

For $d \leqslant 2$ considere the exact triangle

$$
\left.\left.\left.\tau_{\leqslant d} R \pi_{*} \mathbb{Q}_{\ell} / \mathbb{Z}_{\ell}(d)\right) \longrightarrow R \pi_{*} \mathbb{Q}_{\ell} / \mathbb{Z}_{\ell}(d)\right) \longrightarrow R^{d+1} \pi_{*} \mathbb{Q}_{\ell} / \mathbb{Z}_{\ell}(d)\right)[-d+1] \stackrel{[+1]}{\longrightarrow}
$$

leading to the exact sequence extending the left vertical map of the diagram:

$$
\left.\mathrm{H}_{\mathrm{Zar}}^{d-3}\left(Y, R^{d+1} \pi_{*} \mathbb{Q}_{\ell} / \mathbb{Z}_{\ell}(d)\right)\right) \longrightarrow \mathrm{H}_{\mathrm{Zar}}^{2 d-1}\left(Y, \tau_{\leqslant d} R \pi_{*} \mathbb{Q}_{\ell} / \mathbb{Z}_{\ell}(d)\right) \longrightarrow \mathrm{H}_{\text {ét }}^{2 d-1}\left(Y, \mathbb{Q}_{\ell} / \mathbb{Z}_{\ell}(d)\right) \text {. }
$$

Now, the first group can be identified with the third homology group of the Kato complex, which vanishes by a dimension argument for $d \leqslant 2$.

Therefore, in all cases we see, that the class map is an injection

$$
\mathrm{CH}^{d}(Y)[\ell] \hookrightarrow \mathrm{H}^{2 d}\left(Y, \mathbb{Z}_{\ell}(d)\right)
$$

and by Poincaré duality [Mil80] VI.11.1 for $\ell \neq \operatorname{char}(k))$ and [JSS09], [Mos99], Mil86], resp., for $\ell=\operatorname{char}(k)$, this map can identified with the reciprocity map

$$
\mathrm{CH}_{0}(Y)[\ell] \rightarrow \pi_{1}^{\mathrm{ab}}(Y)(\ell) .
$$

So we get the desired results.

Lemma 1.4.6. $\quad$ 1. Let $f: Y \rightarrow \operatorname{Spec}(k)$ be a normal scheme which is separated, of finite type and geometrically connected over a finite field $k$. The degree map

$$
\begin{aligned}
& \operatorname{deg}: Z_{0}(Y) \quad \rightarrow \quad \mathbb{Z}, \\
& \sum_{y \in Y_{0}} a_{y} \cdot y \mapsto \sum_{y \in Y_{0}} a_{y} \cdot[\kappa(y): k]
\end{aligned}
$$

is then surjective.

2. Let $Y=\bigcup_{v \in I} Y_{v}$ be a connected scheme which is proper over a finite field $k$ such that $Y_{v}$ are closed normal connected subschemes of $Y$. Let $k_{v}:=\mathcal{O}_{Y_{v}}\left(Y_{v}\right)$. The image of the degree map $\mathrm{CH}_{0}(Y) \rightarrow \mathrm{CH}_{0}(k) \cong \mathbb{Z}$ is then given by

$$
\operatorname{im} \operatorname{deg}=\underset{v \in I}{\operatorname{gcd}}\left(\left[k_{v}: k\right]\right) \cdot \mathbb{Z} .
$$


Proof. 1. Consider the commutative diagram

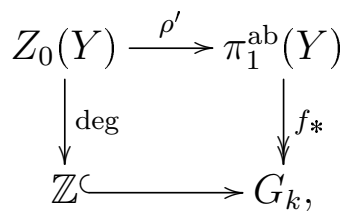

where $G_{k} \cong \hat{\mathbb{Z}}$ and $f_{*}$ is surjective by [Sza09] Prop. 5.5.4 because $Y$ is geometrically connected. Now let $n$ be the natural number given by $n \mathbb{Z}=$ im deg. By 1.4.2 $\rho^{\prime}$ has dense image and therefore $f_{*} \circ \rho^{\prime}$, too. But $n \mathbb{Z}$ is only dense in $\hat{\mathbb{Z}}$ if and only if $n=1$.

2. By the Stein factorisation every $Y_{v}$ is geometrically connected over the finite field $k_{v}$. Now consider the commutative diagram

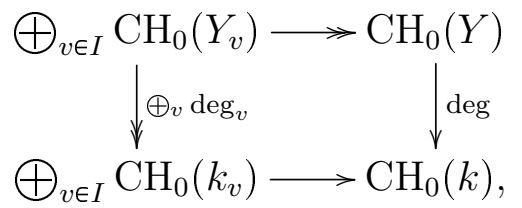

where $\oplus_{v} \operatorname{deg}_{v}$ is surjective by the first point and the bottom map is componentwise on $\mathbb{Z}$ given by multiplying with the degrees $\left[k_{v}: k\right]$ and summing up. So the image of deg equals the image of the bottom map, which is $\operatorname{gcd}_{v \in I}\left(\left[k_{v}: k\right]\right) \cdot \mathbb{Z}$.

Proposition 1.4.7. Let $Y=\bigcup_{v \in I} Y_{v}$ be a proper scheme over a finite field $k$ with closed and connected subschemes $Y_{v} \hookrightarrow Y$ which are smooth over $k$ such that $Y_{v_{0}} \times_{Y} Y_{v_{1}}$ are also smooth over $k$ for all $v_{0}, v_{1} \in I$. Let $n$ be an arbitrary integer and consider the complex

$$
\pi_{1}^{\mathrm{ab}}\left(Y^{[\bullet]}\right) / n: \quad \ldots \longrightarrow \pi_{1}^{\mathrm{ab}}\left(Y^{[1]}\right) / n \stackrel{d_{1}}{\longrightarrow} \pi_{1}^{\mathrm{ab}}\left(Y^{[0]}\right) / n \stackrel{d_{0}}{\longrightarrow} \pi_{1}^{\mathrm{ab}}(Y) / n
$$

with $d_{k}:=\sum_{j=0}^{k}(-1)^{j}\left(\delta_{j}^{k}\right)_{*}$. Then the kernel and the cokernel of the reciprocity map modulo $n$

$$
\rho_{n}: \mathrm{CH}_{0}(Y) / n \rightarrow \pi_{1}^{\mathrm{ab}}(Y) / n
$$

are given by the homology groups of $\pi_{1}^{\mathrm{ab}}\left(Y^{[\bullet]}\right) / n$ :

$$
\begin{aligned}
\operatorname{ker} \rho_{n} & \cong \mathrm{H}_{0}\left(\pi_{1}^{\mathrm{ab}}\left(Y^{[\bullet]}\right) / n\right), \\
\operatorname{coker} \rho_{n} & \cong \mathrm{H}_{-1}\left(\pi_{1}^{\mathrm{ab}}\left(Y^{[\bullet]}\right) / n\right) .
\end{aligned}
$$

Furthermore, we have an exact sequence of finite abelian groups:

$$
\mathrm{H}_{2}(\Gamma, \mathbb{Z} / n) \longrightarrow \mathrm{CH}_{0}(Y) / n \longrightarrow \pi_{1}^{\mathrm{ab}}(Y) / n \longrightarrow \mathrm{H}_{1}(\Gamma, \mathbb{Z} / n) \longrightarrow 0,
$$

where $\Gamma$ is the dual complex to $\left(Y,\left(Y_{i}\right)_{i \in I},(I,<)\right)$. 
Proof. We have a commutative diagramm of complexes

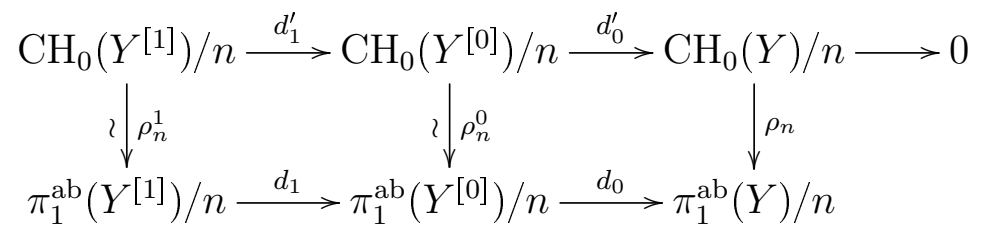

where the first row is exact by 1.3 .3 and the two first vertical maps are isomorphisms by 1.4 .5 since $Y^{[0]}$ and $Y^{[1]}$ are smooth and proper by assumption and $(\hat{\mathbb{Z}} / \mathbb{Z})^{\pi_{0}\left(Y^{[k]}\right)}$ is divisible. By the isomorphisms $\rho_{n}^{0}$ and $\rho_{n}^{1}$ we have coker $\left(d_{1}\right) \cong \mathrm{CH}_{0}(Y) / n$ and

$$
\bar{d}_{0}: \operatorname{coker}\left(d_{1}\right) \rightarrow \pi_{1}^{\mathrm{ab}}(Y) / n
$$

coincide with $\rho_{n}$. Therefore we get

$$
\operatorname{ker}\left(\rho_{n}\right) \cong \operatorname{ker}\left(\bar{d}_{0}\right)=\mathrm{H}_{0}\left(\pi_{1}^{\mathrm{ab}}\left(Y^{[\bullet]}\right) / n\right)
$$

and

$$
\operatorname{coker}\left(\rho_{n}\right)=\operatorname{coker}\left(\bar{d}_{0}\right)=\mathrm{H}_{-1}\left(\pi_{1}^{\mathrm{ab}}\left(Y^{[\bullet]}\right) / n\right) .
$$

And the statement now follows from the abelianized Seifert-Van-Kampen theorem 1.2 .3 with $A=\mathbb{Z} / n$ :

$$
\begin{aligned}
\mathrm{H}_{0}\left(\pi_{1}^{\mathrm{ab}}\left(Y^{[\bullet]}\right) / n\right) & \cong \mathrm{H}_{1}(\Gamma, \mathbb{Z} / n) \\
\mathrm{H}_{-1}\left(\pi_{1}^{\mathrm{ab}}\left(Y^{[\bullet]}\right) / n\right) & \leftarrow \mathrm{H}_{2}(\Gamma, \mathbb{Z} / n) .
\end{aligned}
$$

Remark 1.4.8. 1. The proof of 1.4 .7 and 1.2.3 show that we have

$$
\operatorname{ker} \rho_{n} \cong \operatorname{im}\left(\beta_{\mid}: \operatorname{ker} d_{2} \rightarrow\left(\pi_{1}^{\mathrm{ab}}\left(Y^{[0]}\right) / n\right) / \operatorname{im} d_{1}\right) \text {. }
$$

2. Therefore a non-vanishing of $\beta_{\mid}$results in a non-trivial kernel of the reciprocity map modulo $n$.

3. If we could choose the geometric points of 1.2.1 such that the paths $\gamma_{t, t^{\prime}}$ generate trivial $\alpha_{i j k}^{(f)}$ for all parameters, then we get a vanishing $\beta_{\mid}$and therefore a trivial kernel of the reciprocity map modulo $n$ for every integer $n$.

Notation 1.4.9. For an abelian group $A$ and a set of primes $\mathbb{L}$ let $\mathbb{N}(\mathbb{L})$ be the monoid of all natural numbers which have prime divisiors only in $\mathbb{L}$. We define $A_{\mathbb{L}}$ to be the $\mathbb{L}$-completion

$$
A_{\mathbb{L}}:=\lim _{n \in \mathbb{N}(\mathbb{L})} A / n
$$


and $\hat{A}$ to be

$$
\hat{A}:=\lim _{n \in \mathbb{N}}^{\lim } A / n
$$

the $\hat{\mathbb{Z}}$-completion.

Theorem 1.4.10 (The reciprocity map and its $\mathbb{L}$-completion). Let $\mathbb{L}$ be a set of prime numbers and let $Y=\bigcup_{v \in I} Y_{v}$ be a proper scheme over a finite field $k$ with a finite number of closed and connected subschemes $Y_{v} \hookrightarrow Y$ which are smooth over $k$ such that $Y_{v_{0}} \times_{Y} Y_{v_{1}}$ are also smooth over $k$ for all $v_{0}, v_{1} \in I$. Let $\Gamma$ be the dual complex to $\left(Y,\left(Y_{i}\right)_{i \in I},(I,<)\right)$. Consider the complex

$$
\pi_{1}^{\mathrm{ab}}\left(Y^{[\bullet]}\right)_{\mathbb{L}}: \quad \ldots \stackrel{d_{2}}{\longrightarrow} \pi_{1}^{\mathrm{ab}}\left(Y^{[1]}\right)_{\mathbb{L}} \stackrel{d_{1}}{\longrightarrow} \pi_{1}^{\mathrm{ab}}\left(Y^{[0]}\right)_{\mathbb{L}} \stackrel{d_{0}}{\longrightarrow} \pi_{1}^{\mathrm{ab}}(Y)_{\mathbb{L}}
$$

with $d_{k}:=\sum_{j=0}^{k}(-1)^{j}\left(\delta_{j}^{k}\right)_{*}$ and the reciprocity maps

$$
\begin{aligned}
\rho: \mathrm{CH}_{0}(Y) & \rightarrow \pi_{1}^{\mathrm{ab}}(Y), \\
\rho_{0}: A_{0}(Y) & \rightarrow \pi_{1}^{\mathrm{geo}}(Y), \\
\rho_{\mathbb{L}}: \mathrm{CH}_{0}(Y)_{\mathbb{L}} & \rightarrow \pi_{1}^{\mathrm{ab}}(Y)_{\mathbb{L}}, \\
\hat{\rho}: & \mathrm{CH}_{0}(Y) \rightarrow \pi_{1}^{\mathrm{ab}}(Y)=\pi_{1}^{\mathrm{ab}}(Y),
\end{aligned}
$$

where $A_{0}(Y)$ and $\pi_{1}^{\text {geo }}(Y)$ are the kernels of the corresponding degree maps.

1. Then the kernel of $\rho_{\mathbb{L}}$ is a finite abelian group and a factorgroup of $\mathrm{H}_{2}\left(\Gamma, \mathbb{Z}_{\mathbb{L}}\right)$ and is given by:

$$
\operatorname{ker} \rho_{\mathbb{L}} \cong \mathrm{H}_{0}\left(\pi_{1}^{\mathrm{ab}}\left(Y^{[\bullet]}\right)_{\mathbb{L}}\right) .
$$

The cokernel of $\rho_{\mathbb{L}}$ is given by:

$$
\operatorname{coker} \rho_{\mathbb{L}} \cong \mathrm{H}_{-1}\left(\pi_{1}^{\mathrm{ab}}\left(Y^{[\bullet]}\right)_{\mathbb{L}}\right) \cong \mathrm{H}_{1}\left(\Gamma, \mathbb{Z}_{\mathbb{L}}\right) .
$$

Therefore, we have an exact sequence of finitely generated $\mathbb{Z}_{\mathbb{L}}$-modules:

$$
\mathrm{H}_{2}\left(\Gamma, \mathbb{Z}_{\mathbb{L}}\right) \longrightarrow \mathrm{CH}_{0}(Y)_{\mathbb{L}} \stackrel{\rho_{\mathbb{L}}}{\longrightarrow} \pi_{1}^{\mathrm{ab}}(Y)_{\mathbb{L}} \longrightarrow \mathrm{H}_{1}\left(\Gamma, \mathbb{Z}_{\mathbb{L}}\right) \longrightarrow 0 .
$$

2. For every set of primes $\mathbb{L}$ with $\# A_{0}(Y) \in \mathbb{N}(\mathbb{L})$ we have:

$$
\operatorname{ker} \rho_{\mathbb{L}}=\operatorname{ker} \rho_{0}=\operatorname{ker} \rho=\operatorname{ker} \hat{\rho} \cong \mathrm{H}_{0}\left(\pi_{1}^{\mathrm{ab}}\left(Y^{[\bullet]}\right)\right) \text {. }
$$

3. The cokernels of $\rho$ and $\hat{\rho}$ are given by:

$$
\begin{aligned}
& \text { coker } \hat{\rho} \cong \mathrm{H}_{-1}\left(\pi_{1}^{\mathrm{ab}}\left(Y^{[\bullet]}\right)\right) \cong \mathrm{H}_{1}(\Gamma, \hat{\mathbb{Z}}), \\
& \operatorname{coker} \rho \cong(\hat{\mathbb{Z}} / \mathbb{Z})^{\pi_{0}(Y)} \oplus \mathrm{H}_{1}(\Gamma, \hat{\mathbb{Z}}) .
\end{aligned}
$$

Therefore, we have an exact sequence of abelian groups:

$$
\mathrm{H}_{2}(\Gamma, \hat{\mathbb{Z}}) \longrightarrow \mathrm{CH}_{0}(Y) \stackrel{\rho}{\longrightarrow} \pi_{1}^{\mathrm{ab}}(Y) \longrightarrow(\hat{\mathbb{Z}} / \mathbb{Z})^{\pi_{0}(Y)} \oplus \mathrm{H}_{1}(\Gamma, \hat{\mathbb{Z}}) \longrightarrow 0,
$$


Proof. For the $\mathbb{L}$-completion we have the analogue results from 1.4 .7 by taking the inverse limit with the additional information that for a finitely generated abelian group $A$ we have $A_{\mathbb{L}}=A \otimes_{\mathbb{Z}} \mathbb{Z}_{\mathbb{L}}$, so that the universal coefficent theorem

$$
0 \longrightarrow \mathrm{H}_{i}(\Gamma, \mathbb{Z}) \otimes_{\mathbb{Z}} \mathbb{Z}_{\mathbb{L}} \longrightarrow \mathrm{H}_{i}\left(\Gamma, \mathbb{Z}_{\mathbb{L}}\right) \longrightarrow \operatorname{Tor}_{1}^{\mathbb{Z}}\left(\mathrm{H}_{i-1}(\Gamma, \mathbb{Z}), \mathbb{Z}_{\mathbb{L}}\right) \longrightarrow 0,
$$

gives that $\mathrm{H}_{i}\left(\Gamma, \mathbb{Z}_{\mathbb{L}}\right)=\mathrm{H}_{i}(\Gamma, \mathbb{Z})_{\mathbb{L}}$, because $\mathbb{Z}_{\mathbb{L}}$ is torsionfree and therefore we get $\operatorname{Tor}_{1}^{\mathbb{Z}}\left(\mathrm{H}_{i-1}(\Gamma, \mathbb{Z}), \mathbb{Z}_{\mathbb{L}}\right)=0$. ker $\rho_{\mathbb{L}}$ is finite because it lies in the kernel of the degree map (see diagram below), which is finite by 1.3.6. With 1.4.6 we have the commuative and exact diagramm

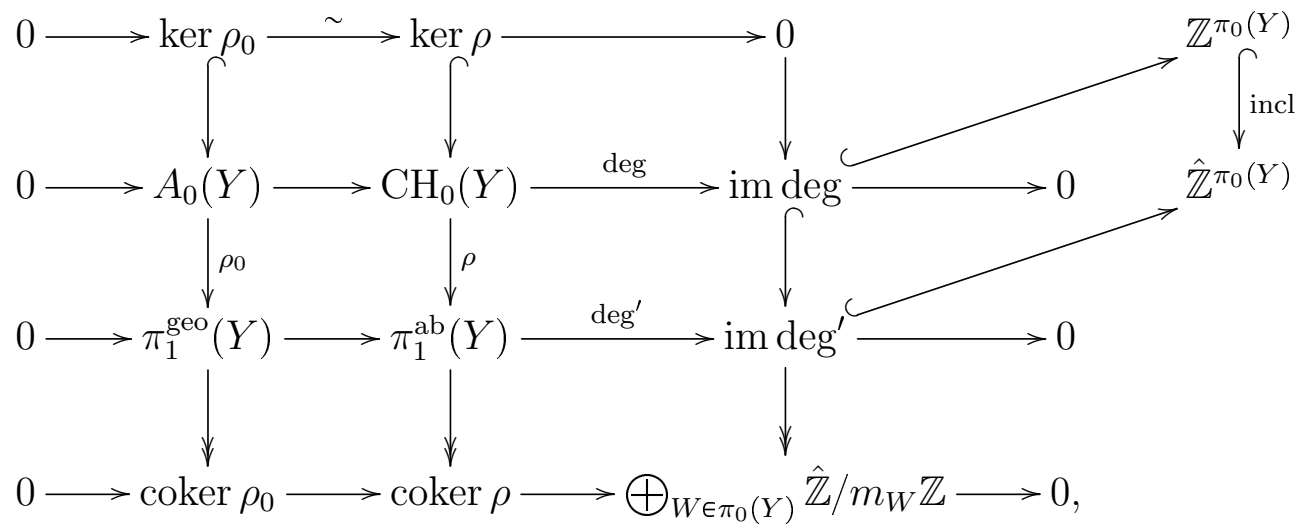

where $m_{W}:=\operatorname{gcd}_{Y_{v} \subseteq W}\left(\left[k_{v}: k_{W}\right]\right)$ and $k_{v}:=\mathcal{O}_{Y_{v}}\left(Y_{v}\right)$ and $k_{W}:=\mathcal{O}_{W}(W)$.

Taking the $\hat{\mathbb{Z}}$-completion of this diagram, we see that the two middle horizontal lines stay exact and that $\operatorname{ker} \rho_{0} \cong \operatorname{ker} \hat{\rho}$. Note that $A_{0}(Y)$ and $\pi_{1}^{\text {geo }}(Y)$ are profinite groups and do not change under $\hat{\mathbb{Z}}$-completion, i.e. we have $\hat{\rho}_{0}=\rho_{0}$. Comparing with the original bottom line sequence we get a commutative diagram of exact sequences

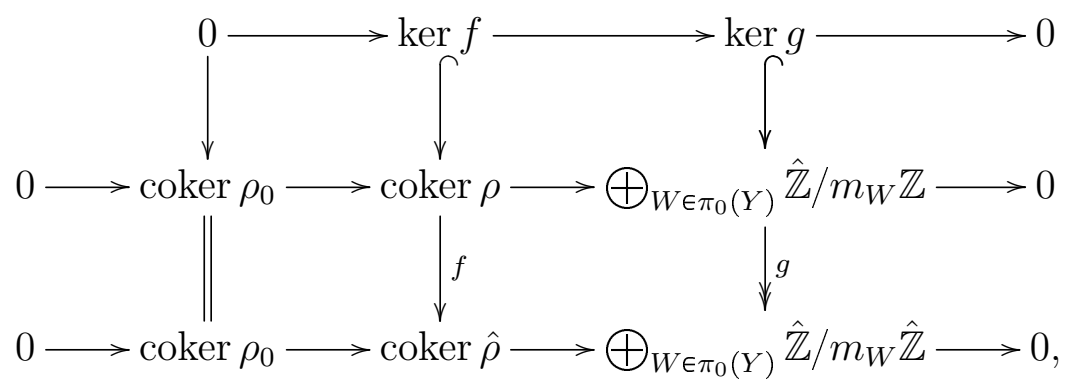

from which follows that $f$ is surjective, $\operatorname{ker} f \cong \operatorname{ker} g \cong(\hat{\mathbb{Z}} / \mathbb{Z})^{\pi_{0}(Y)}$, which is a divisible group. We therefore get an isomorphism

$$
\operatorname{coker} \rho \cong(\hat{\mathbb{Z}} / \mathbb{Z})^{\pi_{0}(Y)} \oplus \operatorname{coker} \hat{\rho} \text {. }
$$


Now let $m \in \mathbb{N}(\mathbb{L})$ be an integer with $m \cdot A_{0}(Y)=0$ and $m \cdot \pi_{1}^{\text {geo }}(Y)_{\mathbb{L} \text {-tors }}=0$, which exists by assumption and since $\pi_{1}^{\text {geo }}(Y)$ is finitely generated abelian pro-finite group. $\rho_{0}$ then factors as

$$
\rho_{0}: A_{0}(Y) \longrightarrow \pi_{1}^{\text {geo }}(Y)_{\mathbb{L}-\text { tors }} \hookrightarrow \pi_{1}^{\text {geo }}(Y)
$$

Since $\pi_{1}^{\text {geo }}(Y) / \pi_{1}^{\text {geo }}(Y)_{\mathbb{L} \text {-tors }}$ is $\mathbb{L}$-torsionfree and $m \in \mathbb{N}(\mathbb{L})$ we get an injection

$$
\pi_{1}^{\text {geo }}(Y)_{\mathbb{L} \text {-tors }} / m \hookrightarrow \pi_{1}^{\text {geo }}(Y) / m,
$$

and therefore a factorisation modulo $m$ :

$$
\rho_{0, m}: A_{0}(Y) / m \longrightarrow \pi_{1}^{\mathrm{geo}}(Y)_{\mathbb{L}-\text { tors }} / m \hookrightarrow \pi_{1}^{\mathrm{geo}}(Y) / m .
$$

Because $A_{0}(Y) / m=A_{0}(Y)$ and $\pi_{1}^{\text {geo }}(Y)_{\mathbb{L} \text {-tors }} / m=\pi_{1}^{\text {geo }}(Y)_{\mathbb{L} \text {-tors }}$ we have

$$
\operatorname{ker} \rho_{0, m}=\operatorname{ker} \rho_{0}=\operatorname{ker} \rho \text {. }
$$

Now taking the limit over $\mathbb{N}(\mathbb{L})$ shows

$$
\operatorname{ker} \rho_{\mathbb{L}}=\operatorname{ker} \rho_{0}=\operatorname{ker} \rho
$$

for such sets $\mathbb{L}$ and also for the set of all prime numbers, which gives the results for $\hat{\rho}$.

Corollary 1.4.11. Let the setting be like in 1.4.10. Furthermore, assume that all components of $Y, Y^{[1]}$ and $Y^{[0]}$ are geometrically connected over $k$ and $Y^{[0]}$ consists in a way of "geometrically simply connected" components, i.e.

$$
\pi_{1}^{\text {geo }}\left(Y^{[0]}\right):=\operatorname{ker}\left(\pi_{1}^{\mathrm{ab}}\left(Y^{[0]}\right) \stackrel{\operatorname{deg}}{\longrightarrow} G_{k}^{\pi_{0}\left(Y^{[0]}\right)} \cong \hat{\mathbb{Z}}^{\pi_{0}\left(Y^{[0]}\right)}\right)
$$

vanishes. (Note, that this assumption by 1.4 .5 and 1.3 .6 is equivalent to say that $\mathrm{CH}_{0}\left(Y^{[0]}\right)$ is torsion-free.) The kernel of the reciprocity map then vanishes and also modulo $n$ for every integer $n$.

Proof. We have a commutative diagram of complexes:

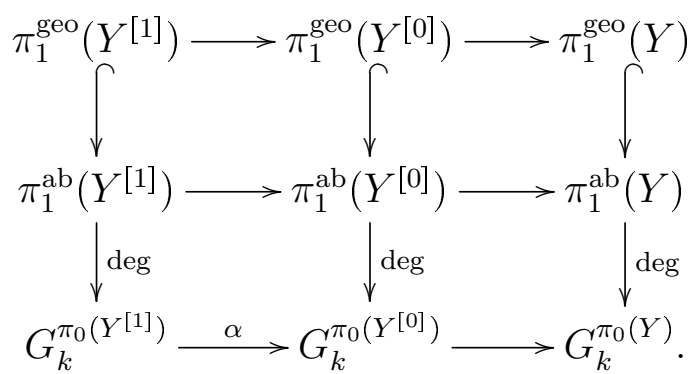


By the assumption of geometrical connectedness the degree maps are surjective. Therefore we have a short exact sequence of complexes:

$$
0 \longrightarrow \pi_{1}^{\mathrm{geo}}\left(Y^{[\bullet]}\right) \longrightarrow \pi_{1}^{\mathrm{ab}}\left(Y^{[\bullet]}\right) \longrightarrow G_{k}^{\pi_{0}\left(Y^{[\bullet]}\right)} \longrightarrow 0
$$

And because $G_{k} \cong \hat{\mathbb{Z}}$ is torsionfree, we get short exact sequences of complexes for every integer $n$ :

$$
0 \longrightarrow \pi_{1}^{\text {geo }}\left(Y^{[\bullet]}\right) / n \longrightarrow \pi_{1}^{\mathrm{ab}}\left(Y^{[\bullet]}\right) / n \longrightarrow(\mathbb{Z} / n)^{\pi_{0}\left(Y^{[\bullet]}\right)} \longrightarrow 0 .
$$

From the long exact sequence follows the exact sequence:

$$
\mathrm{H}_{0}\left(\pi_{1}^{\mathrm{geo}}\left(Y^{[\bullet]}\right) / n\right) \longrightarrow \mathrm{H}_{0}\left(\pi_{1}^{\mathrm{ab}}\left(Y^{[\bullet]}\right) / n\right) \longrightarrow \mathrm{H}_{0}\left((\mathbb{Z} / n)^{\pi_{0}\left(Y^{[\bullet]}\right)}\right),
$$

where the first term vanishes by assumption. For the last term we mention that we have

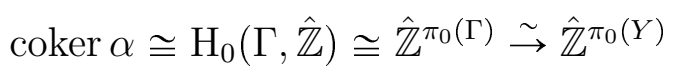

by 1.1.6. The isomorphism above also holds with $\mathbb{Z} / n$-coefficients. Therefore we have $\mathrm{H}_{0}\left((\mathbb{Z} / n)^{\pi_{0}\left(Y^{[\bullet]}\right)}\right)=0$. So $\mathrm{H}_{0}\left(\pi_{1}^{\mathrm{ab}}\left(Y^{[\bullet]}\right) / n\right)$ vanishes. By 1.4 .7 the last term is isomorphic to $\operatorname{ker} \rho_{n}$. So the claim follows. The same way shows that $\operatorname{ker} \rho=0$.

\subsection{Examples}

Here we will give examples of varieties over finite fields, for which we are able to compute the kernel of the reciprocity map explicitly. We will see that by knowing the kernel of the reciprocity map, we can not make easy conclusions to the second homology group of the dual complex, e.g. about vanishing or the size of its torsion parts.

Example 1.5.1. Let $k$ be a finite field and $Y=V_{+}\left(T_{0} \cdot T_{1} \cdot T_{2} \cdot T_{3}\right) \subseteq \mathbb{P}_{k}^{3}=$ $\operatorname{Proj}\left(k\left[T_{0}, \ldots, T_{3}\right]\right)$ be the surface of the projective tetrahedron. The reciprocity map

$$
\rho: \mathrm{CH}_{0}(Y) \rightarrow \pi_{1}^{\mathrm{ab}}(Y)
$$

then has trivial kernel and also the reciprocity maps modulo $n$ for every integer $n$. But we have

$$
\mathrm{H}_{2}\left(\Gamma_{Y}, \mathbb{Z} / n\right) \cong \mathbb{Z} / n \text {. }
$$

So $\mathrm{H}_{2}\left(\Gamma_{Y}, \mathbb{Z} / n\right)$ surjects onto ker $\rho_{n}$, but i.g. does not inject into $\mathrm{CH}_{0}(Y) / n$.

Proof. The calculation of $\mathrm{H}_{2}\left(\Gamma_{Y}, \mathbb{Z} / n\right)$ is clear. The rest follows from 1.4 .11 and the fact that $\pi_{1}\left(\mathbb{P}_{k}^{m}, \bar{x}_{i}\right) \cong G_{k}$, i.e. $\mathbb{P}_{k}^{m}$ is "geometrically simply connected". Note that every intersection of the irreducible components is isomorphic to a $\mathbb{P}_{k}^{m}$. 
Remark 1.5.2. This example can be generalized by the following:

Lemma 1.5.3. Let $k$ be a field and let $i: W \hookrightarrow Z$ be a closed immersion between proper smooth geometrically connected $k$-varieties such that $d:=\operatorname{dim} Z \geqslant 3$, the complement $U=Z \backslash W$ is affine and further assume one of the following properties:

- The natural map $\pi_{1}(Z, \bar{z}) \rightarrow G_{k}$ has a section for a geometric point $\bar{z}$ on $Z$ (which is the case if $Z$ has a k-rational point).

- The cohomology groups $\mathrm{H}^{2}\left(G_{k}, \mathbb{Q} / \mathbb{Z}\right)$ vanish.

Then the push-forward map $i_{*}: \pi_{1}^{\mathrm{ab}}(W) \rightarrow \pi_{1}^{\mathrm{ab}}(Z)$ is an isomorphism.

Proof. For a separably closed field $k$ this is due to [Sat05]. The proof there uses Poincaré duality [Mil80] VI.11.1, the affine Lefschetz theorem Mil80. VI.7.2 and the assumption $\operatorname{dim} \geqslant 3$ to show that $\mathrm{H}_{c}^{i}(U, \mathbb{Q} / \mathbb{Z})[\ell]$ vanishes for $i=1,2$ and $\ell \neq p=\operatorname{char}(k)$. For $\ell=p$ one needs duality results from [JSS09] Thm. 1.6, 1.7, cf. Mos99], Mil86] §1, and the corresponding vanishing results from [Suw95] 2.1 for the cohomology of the logarithmic part of de Rham-Witt sheaves, cf. [Ill79. For an arbitrary field $k$, one base changes with an separable closure $\bar{k}$ of $k$ and uses the homotopy exact sequence from Gro71] IX Thm. 6.1 to get a commutative diagram of exact sequences:

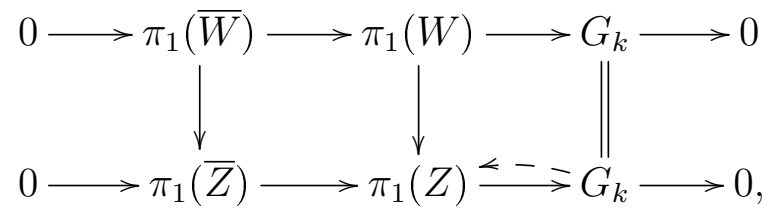

suppressing the geometric points. This induces a commutative diagram of exact sequences:

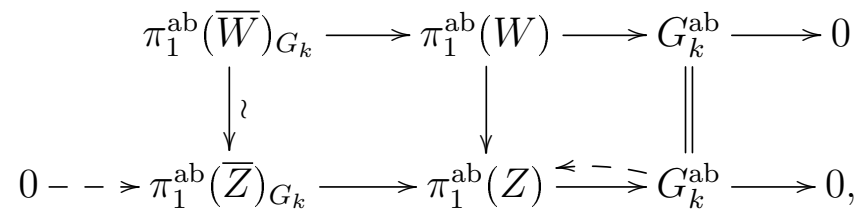

where the injectivity on the left bottom is induced by the section given by assumption or via the Pontyagin dual of the Hochschild-Serre 4-term sequence

$$
0 \longrightarrow \mathrm{H}^{1}\left(G_{k}, \mathbb{Q} / \mathbb{Z}\right) \longrightarrow \mathrm{H}^{1}(Z, \mathbb{Q} / \mathbb{Z}) \longrightarrow \mathrm{H}^{1}(\bar{Z}, \mathbb{Q} / \mathbb{Z})^{G_{k}} \longrightarrow \mathrm{H}^{2}\left(G_{k}, \mathbb{Q} / \mathbb{Z}\right)=0
$$

With the previous result over separably closed fields and the snake lemma one gets the claim. 
Example 1.5.4. Let $k$ be a finite field and $\mathbb{P}_{k}^{d+1}$ be the projective space. Let $f_{1}, \ldots, f_{n}$ be homogeneous irreducible polynomials defining smooth and geometrically connected hypersurfaces in $\mathbb{P}_{k}^{d+1}$, such that $V_{+}\left(f_{i}\right)$ and $V_{+}\left(f_{j}\right)$ intersect smoothly and every connected component of $V_{+}\left(f_{i}, f_{j}\right)$ is geometrically connected (e.g. contains a $k$-rational point), then the reciprocity map $\rho^{Y}$ is injective for $Y:=V_{+}\left(f_{1} \cdots f_{n}\right) \hookrightarrow$ $\mathbb{P}_{k}^{d+1}$.

Proof. If $d<2$, then $Y$ is a union of points or curves, so that $\mathrm{H}_{2}\left(\Gamma_{Y}\right)=0$. For $d \geqslant 2$ we use 1.5 .3 together with $\pi_{1}^{\mathrm{ab}}\left(\mathbb{P}_{k}^{d+1}\right)=G_{k}^{\mathrm{ab}}$ and refer to 1.4 .11 .

Definition 1.5.5 (Simple normal crossing varieties). Let $k$ be a field and $Y$ a equidimensional and separated scheme of finite type over $k . Y$ is called normal crossing variety over $k$, if $Y$ is everywhere étale locally isomorphic to

$$
\operatorname{Spec}\left(k\left[T_{0}, \ldots, T_{d}\right] /\left(T_{0} T_{1} \cdots T_{r}\right)\right),
$$

with $d=\operatorname{dim} Y$ and some $0 \leqslant r \leqslant d$.

A normal crossing variety $Y$ is called simple if any irreducible component of $Y$ is smooth over $k$.

Remark 1.5.6. Simple normal crossing varieties which are proper over a finite field $k$ clearly fulfill 1.4.7 and 1.4.10. For this kind of varieties the results was shown in [JS03] using homology theories and where the $Y_{i}$ are the irreducible components of $Y$. We will repeat this approach in the next chapter.

Example 1.5.7 (cf. [MSA99 Example 4.1, |Sug09 Example 3.2). Let $k$ be a finite field and $n>1$ an integer such that $\operatorname{gcd}(n, 6 \cdot \operatorname{char}(k))=1$ and $k$ contains a primitive $n$-th root of unity $\zeta$. Let $\mathbb{P}_{k}^{3}=\operatorname{Proj}\left(k\left[T_{0}, T_{1}, T_{2}, T_{3}\right]\right)$ be the projektive space and

$$
V:=V_{+}\left(T_{0}^{n}+T_{1}^{n}+T_{2}^{n}+T_{3}^{n}\right) \quad \hookrightarrow \quad \mathbb{P}_{k}^{3}
$$

a Fermat surface and consider the free action on $V$ given by

$$
\tau:\left(T_{0}: T_{1}: T_{2}: T_{3}\right) \mapsto\left(T_{0}: \zeta T_{1}: \zeta^{2} T_{2}: \zeta^{3} T_{3}\right)
$$

Then $X:=V /\langle\tau\rangle$ is a smooth and projective surface. Let

$$
L=V_{+}\left(T_{0}+T_{1}, T_{2}+T_{3}\right) \quad \text { and } \quad L^{\prime}=V_{+}\left(T_{0}+T_{1}, T_{2}+\zeta T_{3}\right)
$$

be two lines on $V$ and $C, C^{\prime}$ be the images in $X . D:=C \cup C^{\prime}$ is then a simple normal crossing divisor on $X$ and $C$ and $C^{\prime}$ meet in two $k$-rational points. We put

$$
Y:=\left(X \times_{k} O\right) \cup\left(X \times_{k} \infty\right) \cup\left(D \times_{k} \mathbb{P}_{k}^{1}\right) \subseteq X \times_{k} \mathbb{P}_{k}^{1}
$$


where $O=(0: 1)$ and $\infty=(1: 0)$ are rational points on $\mathbb{P}_{k}^{1} . Y$ is then a simple normal crossing surface in $X \times{ }_{k} \mathbb{P}_{k}^{1}$ which is projective and geometrically connected over $k$ and the reciprocity map

$$
\rho_{Y}: \mathrm{CH}_{0}(Y) \rightarrow \pi_{1}^{\mathrm{ab}}(Y)
$$

has kernel $\operatorname{ker}\left(\rho_{Y}\right) \cong \mathbb{Z} / n$. Moreover, we have $\operatorname{ker}\left(\rho_{Y \otimes F}\right) \cong \mathbb{Z} / n$ for every finite field extension $F \mid k$.

Now let $C \cap C^{\prime}=\left\{c_{1}, c_{2}\right\}$ and let $\Gamma_{Y}$ be the dual complex to $Y$ associated to a numbering of the irreducible components. We then have:

$$
\begin{aligned}
& \mathrm{H}_{0}\left(\Gamma_{Y}, \mathbb{Z}\right)=\mathbb{Z}, \\
& \mathrm{H}_{1}\left(\Gamma_{Y}, \mathbb{Z}\right)=0, \\
& \mathrm{H}_{2}\left(\Gamma_{Y}, \mathbb{Z}\right) \cong \mathbb{Z},
\end{aligned}
$$

and therefore for every integer $m$ :

$$
\begin{aligned}
& \mathrm{H}_{0}\left(\Gamma_{Y}, \mathbb{Z} / m\right)=\mathbb{Z} / m, \\
& \mathrm{H}_{1}\left(\Gamma_{Y}, \mathbb{Z} / m\right)=0, \\
& \mathrm{H}_{2}\left(\Gamma_{Y}, \mathbb{Z} / m\right) \cong \mathbb{Z} / m .
\end{aligned}
$$

Proof. For the first statements see Sug09 Example 3.2. For the homology groups we mention the following:

$Y, Y^{[0]}$ resp., has 4 irreducible components:

$$
\begin{aligned}
& Y_{1}=X \times_{k} O \\
& Y_{2}=C \times_{k} \mathbb{P}_{k}^{1}, \\
& Y_{3}=C^{\prime} \times_{k} \mathbb{P}_{k}^{1} \\
& Y_{4}=X \times_{k} \infty
\end{aligned}
$$

There are 6 connected components in $Y^{[1]}$ :

$$
\begin{aligned}
& Y_{12}=Y_{1} \cap Y_{2}=C \times{ }_{k} O, \\
& Y_{13}=Y_{1} \cap Y_{3}=C^{\prime} \times{ }_{k} O, \\
& Y_{23}^{1} \text { in } Y_{2} \cap Y_{3}: c_{1} \times{ }_{k} \mathbb{P}_{k}^{1}, \\
& Y_{23}^{2} \text { in } Y_{2} \cap Y_{3}: c_{2} \times{ }_{k} \mathbb{P}_{k}^{1}, \\
& Y_{24}=Y_{2} \cap Y_{4}=C \times{ }_{k} \infty, \\
& Y_{34}=Y_{3} \cap Y_{4}=C^{\prime} \times{ }_{k} \infty .
\end{aligned}
$$

And there are 4 connected components in $Y^{[2]}$ :

$$
\begin{array}{llll}
Y_{123}^{1} & \text { in } Y_{1} \cap Y_{2} \cap Y_{3}: & c_{1} \times_{k} O, \\
Y_{123}^{2} \text { in } Y_{1} \cap Y_{2} \cap Y_{3} & : & c_{2} \times{ }_{k} O, \\
Y_{234}^{1} \text { in } Y_{2} \cap Y_{3} \cap Y_{4} & : & c_{1} \times_{k} \infty, \\
Y_{234}^{2} & \text { in } Y_{2} \cap Y_{3} \cap Y_{4}: & : & c_{2} \times_{k} \infty .
\end{array}
$$

We therefore can compute the homology groups $\mathrm{H}_{i}\left(\Gamma_{Y}, \mathbb{Z}\right)$ : 
$\mathrm{H}_{0}$ : We have:

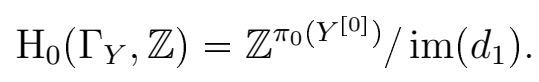

And $d_{1}: \mathbb{Z}^{\pi_{0}\left(Y^{[1]}\right)} \rightarrow \mathbb{Z}^{\pi_{0}\left(Y^{[0]}\right)}$ is given by the matrix

$$
\left(\begin{array}{r|rrrrrr} 
& Y_{12} & Y_{13} & Y_{23}^{1} & Y_{23}^{2} & Y_{24} & Y_{34} \\
\hline Y_{1} & 1 & 1 & 0 & 0 & 0 & 0 \\
Y_{2} & -1 & 0 & 1 & 1 & 1 & 0 \\
Y_{3} & 0 & -1 & -1 & -1 & 0 & 1 \\
Y_{4} & 0 & 0 & 0 & 0 & -1 & -1 \\
\hline & * & & * & & & *
\end{array}\right)
$$

And this matrix has rank 3 given by the marked (*) columns. Adjoining the element given by the column $(0,0,1,0)$ we get a basis of $\mathbb{Z}^{\pi_{0}\left(Y^{[0]}\right)}$. Therefore $\mathrm{H}_{0}\left(\Gamma_{Y}, \mathbb{Z}\right)$ is torsionfree of rank $4-3=1$ and we have

$$
\mathrm{H}_{0}\left(\Gamma_{Y}, \mathbb{Z}\right)=0
$$

The kernel of $d_{1}$ then has rank $6-3=3$.

$\mathrm{H}_{1}$ : By definition we have:

$$
\mathrm{H}_{1}\left(\Gamma_{Y}, \mathbb{Z}\right)=\operatorname{ker}\left(d_{1}\right) / \operatorname{im}\left(d_{2}\right)
$$

And $d_{2}: \mathbb{Z}^{\pi_{0}\left(Y^{[2]}\right)} \rightarrow \mathbb{Z}^{\pi_{0}\left(Y^{[1]}\right)}$ is given by the first matrix:

$$
\left(\begin{array}{r|rr|rr} 
& Y_{123}^{1} & Y_{234}^{1} & Y_{123}^{2} & Y_{234}^{2} \\
\hline Y_{12} & -1 & 0 & -1 & 0 \\
Y_{13} & 1 & 0 & 1 & 0 \\
\hline Y_{23}^{1} & -1 & -1 & 0 & 0 \\
Y_{23}^{2} & 0 & 0 & -1 & -1 \\
\hline Y_{24} & 0 & 1 & 0 & 1 \\
Y_{34} & 0 & -1 & 0 & -1 \\
\hline & * & * & * &
\end{array}\right) \quad\left(\begin{array}{rrr} 
& \\
\hline 1 & 0 & 0 \\
0 & 1 & 0 \\
\hline 0 & 0 & 0 \\
0 & 0 & 0 \\
\hline 0 & 0 & 1 \\
0 & 0 & 0 \\
\hline & &
\end{array}\right.
$$

The difference between the two columns in one block are always the same. But the marked $\left(^{*}\right)$ columns are linearly independent. Therefore the matrix has rank 3. Adjoining the three elements given by the columns of the second matrix we get a basis of $\mathbb{Z}^{\pi_{0}\left(Y^{[1]}\right)}$. So $\mathrm{H}_{1}\left(\Gamma_{Y}, \mathbb{Z}\right)$ is torsionfree of rank $3-3=0$ and we have

$$
\mathrm{H}_{1}\left(\Gamma_{Y}, \mathbb{Z}\right)=0 .
$$

The kernel of $d_{2}$ has rank $4-3=1$ and is generated by the element $Y_{123}^{1}-$ $Y_{234}^{1}-Y_{123}^{2}+Y_{234}^{2}$. 
$\mathrm{H}_{2}$ : Because we are only working with surfaces we have

$$
\mathrm{H}_{2}\left(\Gamma_{Y}, \mathbb{Z}\right)=\operatorname{ker}\left(d_{2}\right) \cong \mathbb{Z}
$$

The homology groups with coefficients in $\mathbb{Z} / m$ can be computed by the homology groups with coefficients in $\mathbb{Z}$ by the universal coefficient theorem observing that all $\mathrm{H}_{i}\left(\Gamma_{Y}, \mathbb{Z}\right)$ are torsionfree.

Remark 1.5.8. 1.5.7 shows that the groups $\mathrm{H}_{2}\left(\Gamma_{Y}, \mathbb{Z}\right)$ and $\mathrm{H}_{2}\left(\Gamma_{Y}, \hat{\mathbb{Z}}\right)$ are torsionfree, but the reciprocity map and the reciprocity map modulo $n$ has kernel $\mathbb{Z} / n$. Therefore the kernel is not given by the torsion part of $\mathrm{H}_{2}\left(\Gamma_{Y}, \mathbb{Z}\right)$ or $\mathrm{H}_{2}\left(\Gamma_{Y}, \hat{\mathbb{Z}}\right)$. 


\section{Homology theories}

Homology theories are the main tool in this thesis to describe class field theory in a functorial way. With this method one is able to relate class field theory of a variety over local fields with class field theory over finite fields, which will be done in the next chapter. Therefore we will recapitulate the definitions and results from [JS03. The main goal of this chapter is to understand the niveau spectral sequence associated to the étale homology theory of a scheme $\mathfrak{X}$ over a henselian discrete valuation ring with finite residue field which will relate the two fibres of $\mathfrak{X}$ to each other.

\subsection{Homology theories and their niveau spectral sequence}

Here we will give the definition and properties of homology theories of Borel-Moore type following [JS03. The main point is that for such a homology theory there always exists a niveau spectral sequence.

Definition 2.1.1. Let $\mathcal{C}$ be category of noetherian schemes such that for any object $X$ in $\mathcal{C}$, every closed immersion $Y \hookrightarrow X$ and every open immersion $U \hookrightarrow X$ is a morphism in $\mathcal{C}$. Let $\mathcal{C}_{*}$ be the category with the same objects as $\mathcal{C}$, but where morphisms are just the proper maps in $\mathcal{C}$.

1. A homology theory on $\mathcal{C}$ is a sequence of covariant functors

$$
\mathrm{H}_{a}\left({ }_{-}\right): \mathcal{C}_{*} \rightarrow \mathcal{A} b
$$

from $\mathcal{C}_{*}$ to the category of abelian groups with $a \in \mathbb{Z}$ satisfying the following conditions:

a) For each open immersion $j: V \hookrightarrow X$ in $\mathcal{C}$, there is a map

$$
j^{*}: \mathrm{H}_{a}(X) \rightarrow \mathrm{H}_{a}(V),
$$

associated to $j$ in a (contravariant) functorial way.

b) If $i: Y \hookrightarrow X$ is a closed immersion in $\mathcal{C}$, with open complement $j: V \hookrightarrow$ $X$, there is long exaxt sequence (called localisation or excision sequence)

$$
\ldots \stackrel{\delta}{\longrightarrow} \mathrm{H}_{a}(Y) \stackrel{i_{*}}{\longrightarrow} \mathrm{H}_{a}(X) \stackrel{j^{*}}{\longrightarrow} \mathrm{H}_{a}(V) \stackrel{\delta}{\longrightarrow} \mathrm{H}_{a-1}(Y) \longrightarrow \ldots
$$


with connecting morphisms $\delta$ and which is functorial with respect to proper maps and open immersions, in an obvious way.

2. A morphism of homology theories $\mathrm{H}$ and $\mathrm{H}^{\prime}$ is a morphism $\phi: \mathrm{H} \rightarrow \mathrm{H}^{\prime}$ of functors on $\mathcal{C}_{*}$ which is compatible with the properties above.

Example 2.1.2 (Étale homology theory). Let $S$ be a noetherian scheme and let $\mathcal{C}=\operatorname{Sch}_{\mathrm{sft}}(S)$ be category of schemes which are separated and of finite type over $S$. Let $\Lambda$ be a bounded complex of étale sheaves on $S$. Then one gets the étale homology theory over $S$ with values in $\Lambda$ by putting

$$
\mathrm{H}_{a}^{\Lambda}(T):=\mathrm{H}_{a}(T \mid S ; \Lambda):=\mathrm{H}^{-a}\left(T_{e ́ t}, R f^{!} \Lambda\right)
$$

for a scheme $f: T \rightarrow S$ in $\mathcal{C}$. Here $R f^{!}$is the right adjoint of $R f_{!}$(see AGV73) XVIII 3.1.4).

Proof. For the functorialities see [JS03] example 2.2.

Lemma 2.1.3 (Mayer-Vietoris sequence). Let $\mathrm{H}$ be a homology theory like in 2.1.1 and let $X=X_{1} \cup X_{2}$ be the union of two closed subschemes $i_{\mu}: X_{\mu} \hookrightarrow X$, and let $k_{\mu}: X_{1} \cap X_{2} \hookrightarrow X_{\mu}$ be the closed immersions of the scheme-theoretic intersection. Then there is a long exact Mayer-Vietoris sequence:

$$
\left.\cdots \stackrel{\delta}{\rightarrow} \mathrm{H}_{a}\left(X_{1} \cap X_{2}\right) \stackrel{\left(k_{1 *},-k_{2 *}\right.}{\longrightarrow}\right) \mathrm{H}_{a}\left(X_{1}\right) \oplus \mathrm{H}_{a}\left(X_{2}\right) \stackrel{i_{1 *}+i_{2} *}{\longrightarrow} \mathrm{H}_{a}(X) \stackrel{\delta}{\rightarrow} \mathrm{H}_{a-1}\left(X_{1} \cap X_{2}\right) \rightarrow \ldots
$$

which is functorial with respect to proper maps, excision sequences and morphisms of homology theories, in the natural way.

Proof. The exact sequence is induced from the commutative diagram of excision sequences by diagram chasing:

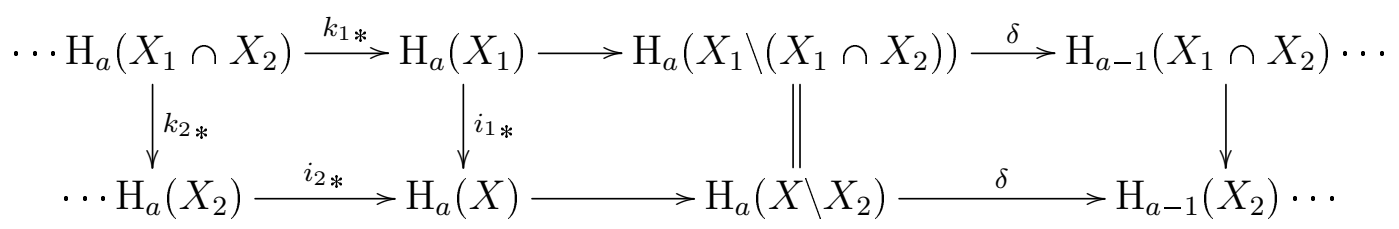

And functorialities are clear from the functoriality of this diagram.

Lemma 2.1.4. Let $\mathrm{H}$ be a homology theory like in 2.1.1 and let $Y, Z \subseteq X$ be closed subschemes with open complements $U, V \subseteq X$. Then we get an infinite diagram of 
localisation sequences

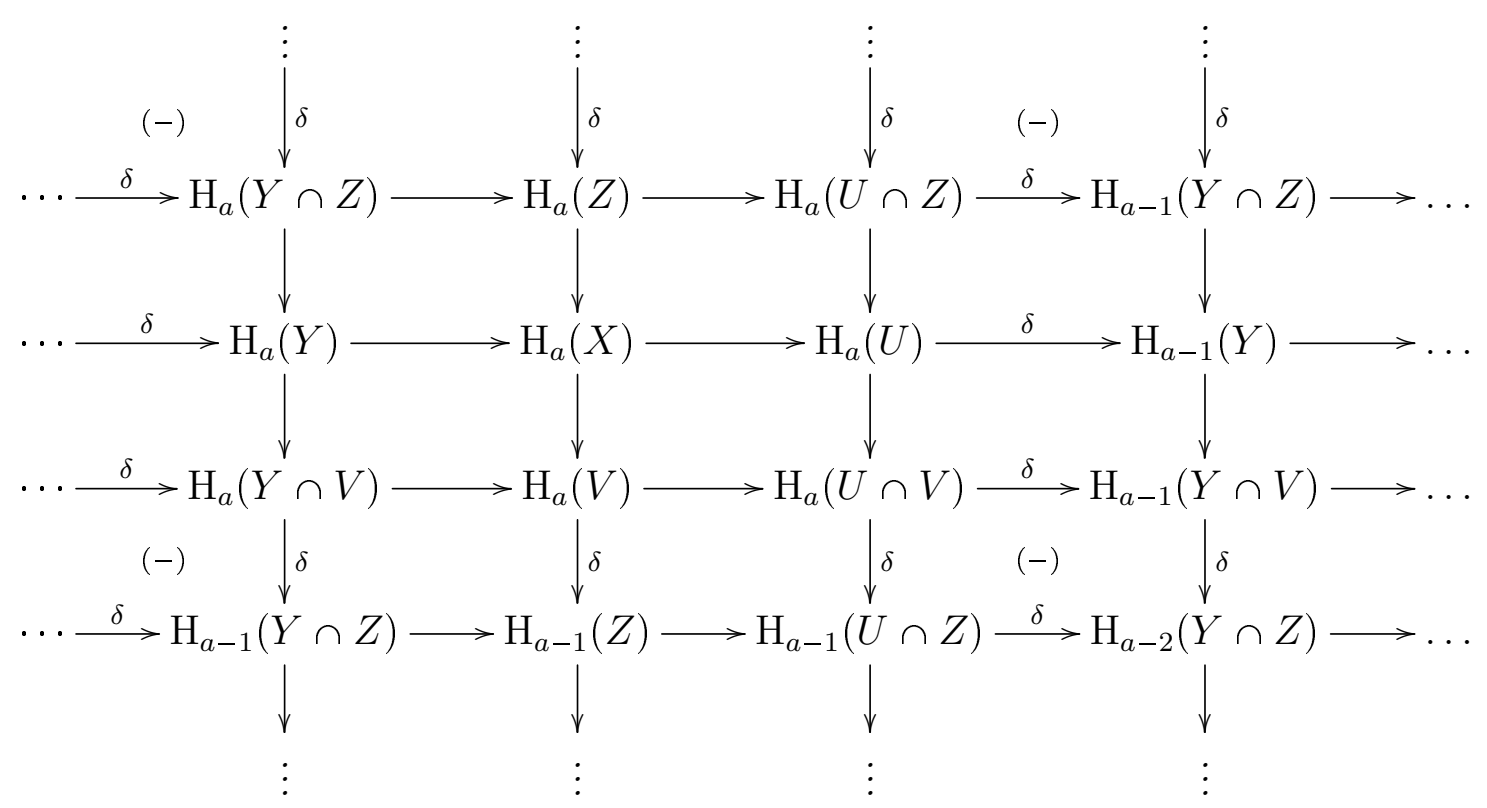

which commutes, except for the squares marked (-), which anticommute.

Proof. We only have to show the anticommutativity of the squares marked. The rest follows from the functoriality in 2.1.1. Since $(Y \cup Z) \backslash(Y \cap Z)=(Y \backslash Z) \coprod(Z \backslash Y)=$ $(Y \cap V) \amalg(U \cap Z)$, from 2.1.3 we have an isomorphism

$$
\mathrm{H}_{a-1}((Y \cup Z) \backslash(Y \cap Z)) \cong \mathrm{H}_{a-1}(Y \cup V) \oplus \mathrm{H}_{a-1}(U \cup Z) .
$$

With $X \backslash(Y \cup Z)=U \cap V$ we get a commuative diagram of localisation sequences:

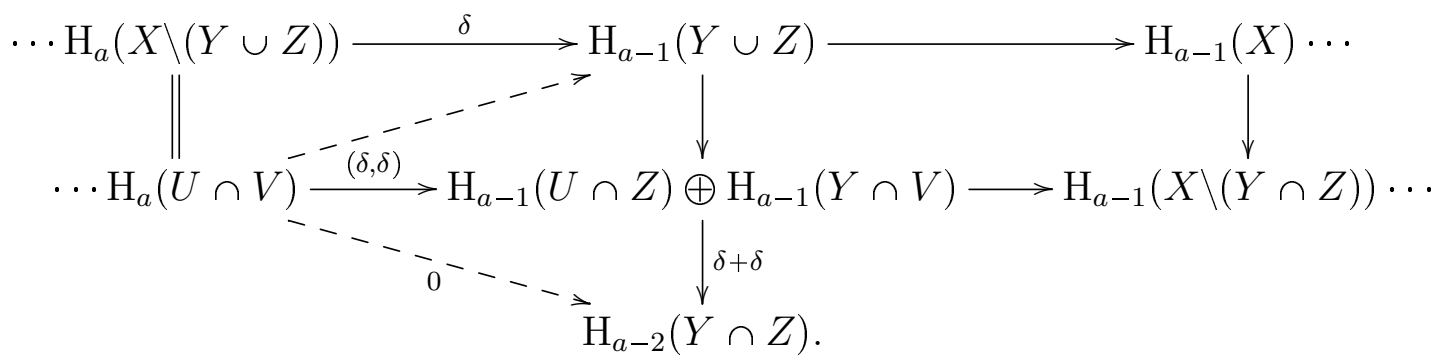

Therefore $(\delta+\delta) \circ(\delta, \delta)$ factors throught $\mathrm{H}_{a-1}(Y \cup Z)$ and we get $(\delta+\delta) \circ(\delta, \delta)=0$, hence the claim.

Corollary 2.1.5. Let $\mathrm{H}$ be a homology theory on a category $\mathcal{C}$ of schemes over $X$ and $W \hookrightarrow X$ be an immersion. Then the groups

$$
\mathrm{H}_{a}^{(W)}(T):=\mathrm{H}_{a}\left(T \times_{X} W\right)
$$

again define a homology theory on $\mathcal{C}$. Furthermore, let $i: Y \hookrightarrow X$ be a closed immersion with open complement $j: U \hookrightarrow X$, then: 
1. $i$ defines a morphism of homology theories $i_{*}: \mathrm{H}^{(Y)} \rightarrow \mathrm{H}$ on $\mathcal{C}$,

2. $j$ defines a morphism of homology theories $j^{*}: \mathrm{H} \rightarrow \mathrm{H}^{(U)}$ on $\mathcal{C}$,

3. the maps $\delta: \mathrm{H}_{a}\left(T \times_{X} U\right) \rightarrow \mathrm{H}_{a-1}\left(T \times_{X} Y\right)$ define a morphism of homology theories $\Delta: \mathrm{H}^{(U)} \rightarrow \mathrm{H}^{(Y)}[-1]$ on $\mathcal{C}$, where the term $[N]$ means that the homology theory is shifted by $N$ and all connecting morphisms $\delta^{(Y)}$ are multiplied by $(-1)^{N}$, i.e. $(-1)$ in that case.

Proof. The statements follow from 2.1.1 and 2.1.4.

Theorem 2.1.6 (Niveau spectral sequence). Let $\mathrm{H}$ be a homology theory on $\mathcal{C}$. Then we have:

1. For every object $X$ in $\mathcal{C}$ there is a spectral sequence of homological type, called niveau spectral sequence:

$$
E_{r, q}^{1}(X)=\bigoplus_{x \in X_{r}} \mathrm{H}_{r+q}(x) \quad \Longrightarrow \quad \mathrm{H}_{r+q}(X) .
$$

Here $X_{r}=\{x \in X \mid \operatorname{dim} \overline{\{x\}}=r\}$ and

$$
\mathrm{H}_{a}(x)=\lim _{x \in V \subseteq\{x\}} \mathrm{H}_{a}(V)
$$

for $x \in X$, where the direct limit is taken over all open non-empty subschemes $V \subseteq \overline{\{x\}}$ and all (descending) inclusion maps.

2. The niveau spectral sequence is covariant with respect to proper maps in $\mathcal{C}$ and contraviant with respect to open immersions.

3. Every morphism $\phi: \mathrm{H} \rightarrow \mathrm{H}^{\prime}$ between homology theories on $\mathcal{C}$ induces a morphism between the associated niveau spectral sequences.

Construction. The construction is analogue to [BO74] $\S 3$ :

- For every scheme $T$ in $\mathcal{C}$ define

$$
\begin{aligned}
\mathcal{Z}_{r}(T) & :=\{Z \subseteq T \text { closed subset } \mid \operatorname{dim} Z \leqslant r\}, \\
\mathcal{Z}_{r, r-1}(T) & :=\left\{\left(Z, Z^{\prime}\right) \in \mathcal{Z}_{r}(T) \times \mathcal{Z}_{r-1}(T) \mid Z \supseteq Z^{\prime}\right\},
\end{aligned}
$$

ordered by inclusion, i.e.:

$$
\begin{aligned}
Z \geqslant W & : \Longleftrightarrow Z \supseteq W, \\
\left(Z, Z^{\prime}\right) \geqslant\left(W, W^{\prime}\right) & : \Longleftrightarrow Z \supseteq W \text { and } Z^{\prime} \supseteq W^{\prime} .
\end{aligned}
$$

These make $\mathcal{Z}_{r}(T)$ and $\mathcal{Z}_{r, r-1}(T)$ into directed sets. An upper bound for two elements is given by the union and the pair of the unions, respectively. 
- For $\left(W, W^{\prime}\right) \leqslant\left(Z, Z^{\prime}\right)$ in $\mathcal{Z}_{r, r-1}(T)$ we get a closed immersion $\iota$ and an open immersion $\nu$ :

$$
W \backslash W^{\prime} \stackrel{\iota}{\hookrightarrow} Z \backslash W^{\prime} \stackrel{\nu}{\longleftrightarrow} Z \backslash Z^{\prime} .
$$

So there are maps

$$
\begin{aligned}
& \nu^{*} \iota_{*} \quad: \quad \mathrm{H}_{a}\left(W \backslash W^{\prime}\right) \stackrel{\iota *}{\rightarrow} \mathrm{H}_{a}\left(Z \backslash W^{\prime}\right) \stackrel{\nu^{*}}{\rightarrow} \mathrm{H}_{a}\left(Z \backslash Z^{\prime}\right), \\
& \lambda_{*}: \mathrm{H}_{a}\left(Z^{\prime}\right) \stackrel{\lambda_{*}}{\rightarrow} \mathrm{H}_{a}(Z),
\end{aligned}
$$

where $\lambda$ denotes the closed immersion $\lambda: Z^{\prime} \hookrightarrow Z$. Therefore we can define:

$$
\begin{aligned}
& \mathrm{H}_{a}\left(\mathcal{Z}_{r}(T)\right):=\lim _{Z \in \mathcal{Z}_{r}(T)} \mathrm{H}_{a}(Z), \\
& \mathrm{H}_{a}\left(\mathcal{Z}_{r, r-1}(T)\right):=\underset{\left(Z, Z^{\prime}\right) \in \mathcal{Z}_{r, r-1}(T)}{\lim _{\longrightarrow}} \mathrm{H}_{a}\left(Z \backslash Z^{\prime}\right),
\end{aligned}
$$

where the direct limit is taken along the (ascending) ordering and the transition maps given by $\lambda_{*}$ and $\nu^{*} \iota_{*}$, respectively, from above.

- For every $\left(W, W^{\prime}\right) \leqslant\left(Z, Z^{\prime}\right)$ in $\mathcal{Z}_{r, r-1}(T)$ there is a commutative diagram of long exact excision sequences:

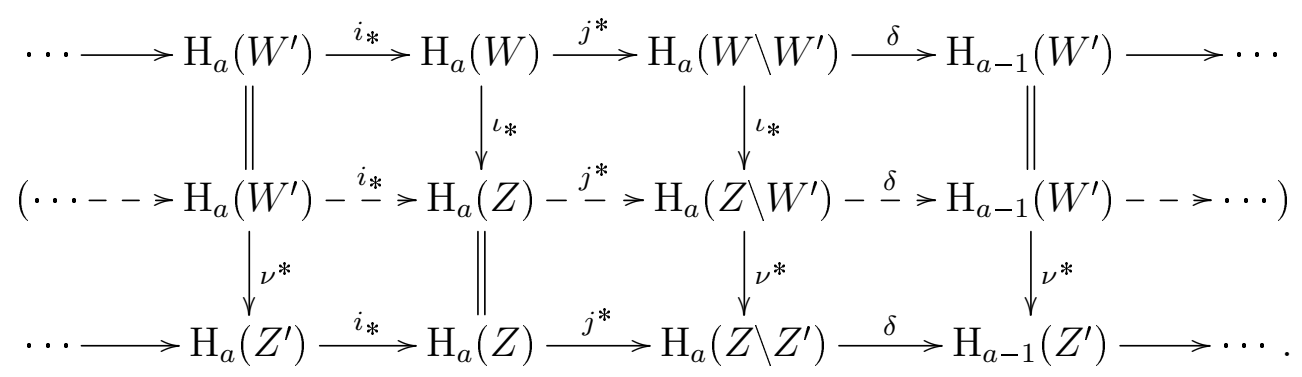

Taking the direct limit over $\mathcal{Z}_{r, r-1}(T)$ gives a long exact sequence:

$$
\cdots \mathrm{H}_{a}\left(\mathcal{Z}_{r-1}(T)\right) \stackrel{i_{*}}{\longrightarrow} \mathrm{H}_{a}\left(\mathcal{Z}_{r}(T)\right) \stackrel{j^{*}}{\longrightarrow} \mathrm{H}_{a}\left(\mathcal{Z}_{r, r-1}(T)\right) \stackrel{\delta}{\longrightarrow} \mathrm{H}_{a-1}\left(\mathcal{Z}_{r-1}(T)\right) \cdots
$$

which is functorial with respect to open immersions and proper maps, in the natural way.

- Summing all these sequences up, we get doubly graded groups

$$
\begin{aligned}
D & :=\bigoplus_{r, q} D_{r, q}:=\bigoplus_{r, q} \mathrm{H}_{r+q}\left(\mathcal{Z}_{r}(T)\right), \\
E & :=\bigoplus_{r, q}^{r} E_{r, q}:=\bigoplus_{r, q} \mathrm{H}_{r+q}\left(\mathcal{Z}_{r, r-1}(T)\right),
\end{aligned}
$$


and an exact triangle

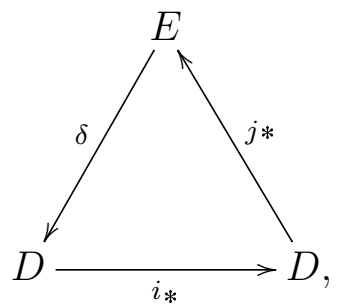

where $i_{*}, j^{*}, \delta$ are homogeneous maps of degrees $(1,-1),(0,0)$ and $(-1,0)$. So $(E, D)$ form an exact $\partial$-couple, from which we get the associated spectral sequence (see [Hu59] Ch. VIII $\S 4+5$ or Wei95] Prp. 5.9.3):

$$
E_{r, q}^{1}=\mathrm{H}_{r+q}\left(\mathcal{Z}_{r, r-1}(T)\right) \quad \Longrightarrow \quad \mathrm{H}_{r+q}(T) .
$$

- Note that $\mathrm{H}_{a}\left(\mathcal{Z}_{r}(T)\right)=0$ for all $r<0$ and that $\mathrm{H}_{a}\left(\mathcal{Z}_{r}(T)\right)=\mathrm{H}_{a}(T)$ for all $r \geqslant d:=\operatorname{dim} T$.

- Also note that $\mathrm{H}_{a}\left(\mathcal{Z}_{r, r-1}(T)\right)=0$ for all $r<0$ and all $r>\operatorname{dim} T$.

- And for all $0 \leqslant r \leqslant \operatorname{dim} T$ we have canonical isomorphisms

$$
\mathrm{H}_{a}\left(\mathcal{Z}_{r, r-1}(T)\right) \cong \bigoplus_{x \in T_{r}} \mathrm{H}_{a}(x)
$$

which comes from the fact that every $w \in \mathrm{H}_{a}\left(\mathcal{Z}_{r, r-1}(T)\right)$ can be represented by an $\tilde{w} \in \mathrm{H}_{a}\left(W \backslash W^{\prime}\right)$ with $W=\bigcup_{x_{i} \in T_{r}} \overline{\left\{x_{i}\right\}}$ and $W^{\prime} \supseteq \bigcup_{i, j}\left(\overline{\left\{x_{i}\right\}} \cap \overline{\left\{x_{j}\right\}}\right)$, so that $W \backslash W^{\prime}$ is a disjoint union of non-empty open subsets of the $\overline{\left\{x_{i}\right\}}$, and that every element $w$ in $\bigoplus_{x \in T_{r}} \mathrm{H}_{a}(x)$ can be represented by finitely many $w_{i} \in \mathrm{H}_{a}\left(V_{i}\right)$ with $V_{i}=\overline{\left\{x_{i}\right\}} \backslash Z_{i}$, so that $W=\bigcup_{x_{i}} \overline{\left\{x_{i}\right\}}$ and $W^{\prime}=\bigcup_{i} Z_{i}$ are the required closed subsets of $T$.

- The $E^{1}$-differentials $d: E_{r, q}^{1} \rightarrow E_{r-1, q}^{1}$ are given by the composites

$$
d: \mathrm{H}_{r+q}\left(\mathcal{Z}_{r, r-1}(T)\right) \stackrel{\delta}{\rightarrow} \mathrm{H}_{r+q-1}\left(\mathcal{Z}_{r-1}(T)\right) \stackrel{j^{*}}{\rightarrow} \mathrm{H}_{r+q-1}\left(\mathcal{Z}_{r-1, r-2}(T)\right)
$$

- The edge isomorphisms

$$
E_{r, q}^{\infty}:=\operatorname{im}\left(j^{*}\right) / j^{*}\left(\operatorname{ker}\left(i_{*}^{\infty}\right)\right) \cong F_{r} E_{r+q} / F_{r-1} E_{r+q}
$$

with $E_{a}:=\mathrm{H}_{a}(T)$ are induced by

$$
E_{r, q}^{1}=\mathrm{H}_{r+q}\left(\mathcal{Z}_{r, r-1}(T)\right) \stackrel{j^{*}}{\longleftarrow} \mathrm{H}_{r+q}\left(\mathcal{Z}_{r}(T)\right) \stackrel{\stackrel{i_{*}^{\infty}}{\longrightarrow}}{\longrightarrow} \mathrm{H}_{r+q}\left(\mathcal{Z}_{\infty}(T)\right)=\mathrm{H}_{r+q}(T),
$$


where the (ascending) filtration on the limit terms are given by

$$
F_{r} \mathrm{H}_{a}(T)=\operatorname{im}\left(\mathrm{H}_{a}\left(\mathcal{Z}_{r}(T)\right) \stackrel{i_{*}^{\infty}}{\longrightarrow} \mathrm{H}_{a}\left(\mathcal{Z}_{\infty}(T)\right)=\mathrm{H}_{a}(T)\right) .
$$

Equivalently, $F_{r} \mathrm{H}_{a}(T)$ is the subgroups of $\mathrm{H}_{a}(T)$ generated by all images $f_{*}$ : $\mathrm{H}_{a}(W) \rightarrow \mathrm{H}_{a}(X)$ where $f: W \rightarrow X$ is a proper map in $\mathcal{C}$ with $\operatorname{dim} f(W) \leqslant r$. Also note, that $F_{r} \mathrm{H}_{a}(T)=0$ for $r<0$ and $F_{r} \mathrm{H}_{a}(T)=\mathrm{H}_{a}(T)$ for $r \geqslant d=$ $\operatorname{dim} T$.

- The functoriality of the niveau spectral sequence comes from that of H.

\subsection{Homology theories over fields}

Here we will give some functoriality properties of the niveau spectral sequence for varieties over fields concerning the first and second page of it.

Proposition 2.2.1. Let $k$ be a field and $S=\operatorname{Spec}(k)$. Let $X$ be separated and of finite type over $k$, and $\mathrm{H}$ be a homology theory on $\operatorname{Sub}(X)$. If $i: W \hookrightarrow X$ is a closed subscheme and $j: U \hookrightarrow X$ the open complement, then the following hold:

1. For all integers $q$, there is an exact sequence of complexes:

$$
0 \longrightarrow E_{\bullet, q}^{1}(W) \stackrel{i_{*}}{\longrightarrow} E_{\bullet, q}^{1}(X) \stackrel{j *}{\longrightarrow} E_{\bullet, q}^{1}(U) \longrightarrow 0,
$$

which is functorial with respect to proper maps and open immersions, in the natural way.

2. The connecting morphisms $\delta: \mathrm{H}_{a}\left(\left(Z \backslash Z^{\prime}\right) \cap U\right) \rightarrow \mathrm{H}_{a}\left(\left(Z \backslash Z^{\prime}\right) \cap W\right)$, for $\left(Z, Z^{\prime}\right) \in \mathcal{Z}_{r, r-1}(X)$ induce a morphism of spectral sequences

$$
\delta: E_{r, q}^{1}(U)^{(-)} \rightarrow E_{r-1, q}^{1}(W),
$$

which is functorial with respect to open and closed immersions, in the natural way. The supscribt (-) means, that all differentials (but not the edge isomorphisms $E_{r, q}^{\infty} \cong F_{r} \mathrm{H}_{r+q} / F_{r-1} \mathrm{H}_{r+q}$ ) are multiplied by $(-1)$.

Proof. See [JS03] Prp. 2.9:

1. Since $X$ is of finite type over a field, we have $X_{r} \cap W=W_{r}$ and $X_{r} \cap U=U_{r}$ and the claim follows from the equality $E_{r, q}^{1}(Z)=\bigoplus_{x \in Z_{r}} \mathrm{H}_{r+q}(x)$.

2. This morphism is induced by the morphism of homology theories $\delta: \mathrm{H}^{(U)}[1] \rightarrow$ $\mathrm{H}^{(W)}$ and the fact that for $Z \in \mathcal{Z}_{r}(U)$ we have that $\bar{Z} \cap W \in \mathcal{Z}_{r-1}(W)$, where $\bar{Z}$ is the closure of $Z$ in $X$. 
Corollary 2.2.2. Let $k$ be a field and $\mathcal{C}$ be a subcategory of $\operatorname{Sch}_{\mathrm{sft}}(\operatorname{Spec}(k))$. For every fixed integer $q$, the family of functors $\left(E_{r, q}^{2}\right)_{r \in \mathbb{Z}}$ defines a homology theory $\tilde{\mathrm{H}}$ on $\mathcal{C}$ given by:

$$
\begin{aligned}
\tilde{\mathrm{H}}_{a}(T) & :=E_{a, q}^{2}(T) \\
& =\mathrm{H}\left(E_{a+1, q}^{1}(T) \stackrel{d^{1}}{\rightarrow} E_{a, q}^{1}(T) \stackrel{d^{1}}{\rightarrow} E_{a-1, q}^{1}(T)\right),
\end{aligned}
$$

with $E_{a, q}^{1}(T)=\bigoplus_{x \in T_{a}} \mathrm{H}_{a+q}(x)$.

Proof. The functoriality for proper maps and open immersions comes from the spectral sequence in 2.2.1, and the localisation sequence is the corresponding long exact sequence of the short exact sequence of complexes in 2.2.1.

Lemma 2.2.3 ( $E^{2}$-homological descent). Let $k$ be a field and $Y=\bigcup_{i=1}^{n} Y_{i}$ be a scheme, separated and of finite type over $k$, which is covered by a finite number of closed subschemes $Y_{i}$. Let $Y^{[r]}$ the $r$-fold intersections of the $Y_{i}$ for $r \geqslant 0$. Let $\mathrm{H}$ be a homology theory on $\operatorname{Sub}(Y)$ and let $\tilde{\mathrm{H}}$ be the homology theory from 2.2.2 for a fixed integer $q$. Then there is a spectral sequence of homological type

$$
{ }^{I} \tilde{E}_{r, t}^{1}=\tilde{\mathrm{H}}_{t}\left(Y^{[r]}\right)=E_{t, q}^{2}\left(Y^{[r]}\right) \Longrightarrow \tilde{\mathrm{H}}_{r+t}(Y)=E_{r+t, q}^{2}(Y) .
$$

Proof. For every $t$ and $q$ the complex

$$
E_{t, q}^{1}\left(Y^{[\bullet]}\right): \quad 0 \leftarrow E_{t, q}^{1}(Y) \leftarrow E_{t, q}^{1}\left(Y^{[0]}\right) \leftarrow E_{t, q}^{1}\left(Y^{[1]}\right) \leftarrow \cdots \leftarrow E_{t, q}^{1}\left(Y^{[n-1]}\right) \leftarrow 0
$$

of the $E^{1}$-terms of the niveau spectral sequence is exact. The maps of the complex are induces by the alternating sum of $i_{j}: Y^{[r]} \rightarrow Y^{[r-1]}$, which are given by inclusion on a $r$-fold intersection into the $(r-1)$-fold intersection omitting the $j$-th component. The exactness then is seen by the equality $E_{t, q}^{1}(T)=\bigoplus_{x \in T_{t}} \mathrm{H}_{t+q}(x)$ and induction on $n$ together with the exact sequence

$$
0 \leftarrow E_{t, q}^{1}\left(Y \cup Y_{n+1}\right) \leftarrow E_{t, q}^{1}(Y) \oplus E_{t, q}^{1}\left(Y_{n+1}\right) \leftarrow E_{t, q}^{1}\left(Y \cap Y_{n+1}\right) \leftarrow 0 .
$$

The $E^{1}$-differential maps then give a bounded complex of exact complexes

$$
E_{0, q}^{1}\left(Y^{[\bullet]}\right) \leftarrow E_{1, q}^{1}\left(Y^{[\bullet]}\right) \leftarrow E_{2, q}^{1}\left(Y^{[\bullet]}\right) \leftarrow \cdots \leftarrow E_{t, q}^{1}\left(Y^{[\bullet]}\right) \leftarrow \cdots \leftarrow E_{d, q}^{1}\left(Y^{[\bullet]}\right),
$$

where $d=\operatorname{dim} Y$. The spectral sequence is then obtained as the hypercohomology spectral sequence of the double complex for the naive filtration. See [Wei95] §5.6.2. 
Example 2.2.4. Let the notation be like in 2.2.3 and let $Y=\bigcup_{i=1}^{n} Y_{i}$ be a normal crossing variety over $k$ of pure dimension $d$. Then we get a first quadrant spectral sequence, where the ${ }^{I} \tilde{E}^{1}$-term of the spectral sequence then looks like:

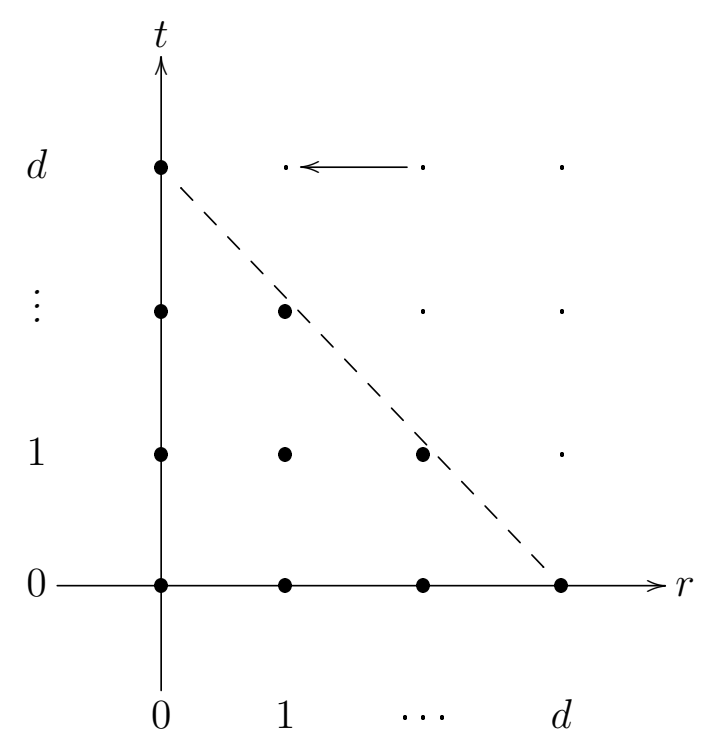

Proof. Since $\operatorname{dim} Y^{[r]} \leqslant d-r$, we have $E_{t, q}^{2}\left(Y^{[r]}\right)=0$ for $t<0$ and $t>d-r$. So the spectral sequence is supported in the area $0 \leqslant t+r \leqslant d$ with $r, t \geqslant 0$.

\subsection{Homology theories over discrete valuation rings}

Here we will show how the niveau spectral sequence of the special and generic fibre of a variety over a discrete valuation ring are connected. The main result is about the second page of those spectral sequences.

Proposition 2.3.1. Let $S=\operatorname{Spec}(A)$ be the spectrum of a discrete valuation ring $A$, let $f: \mathfrak{X} \rightarrow S$ be separated of finite type, and let $\mathrm{H}$ be a homology theory on the category $\operatorname{Sub}(\mathfrak{X})$ of subschemes of $X$. Let $\eta$ and $s$ be the generic point and closed point of $S$, respectively and $X=\mathfrak{X}_{\eta}, Y=\mathfrak{X}_{s}$ the corresponding fibres. With the notations of 2.1.6 we then have:

1. The connecting morphism $\delta: \mathrm{H}_{a}\left(Z \backslash Z^{\prime}\right) \rightarrow \mathrm{H}_{a-1}\left(\left(\bar{Z} \backslash \bar{Z}^{\prime}\right) \cap Y\right)$ for $\left(Z, Z^{\prime}\right) \in$ $\mathcal{Z}_{r, r-1}(X)$ induces a morphism of spectral sequences

$$
\Delta: E_{r, q}^{1}(X)^{(-)} \rightarrow E_{r, q-1}^{1}(Y),
$$

which is functorial with respect to open and closed immersions, in the natural way. The supscribt (-) means, that all differentials (but not the edge isomorphisms $E_{r, q}^{\infty} \cong F_{r} \mathrm{H}_{r+q} / F_{r-1} \mathrm{H}_{r+q}$ ) are multiplied by $(-1)$. 
2. If $f$ is proper, the immersions $i: Y \hookrightarrow \mathfrak{X}, j: X \hookrightarrow \mathfrak{X}$ induce morphisms of spectral sequences

$$
i_{*}: E_{r, q}^{1}(Y) \rightarrow E_{r, q}^{1}(\mathfrak{X}) \quad \text { and } \quad j^{*}: E_{r, q}^{1}(\mathfrak{X}) \rightarrow E_{r-1, q+1}^{1}(X),
$$

such that for all $q$ we get an exact sequence of complexes

$$
0 \longrightarrow E_{\bullet, q}^{1}(Y) \stackrel{i_{*}}{\longrightarrow} E_{\bullet, q}^{1}(\mathfrak{X}) \stackrel{j *}{\longrightarrow} E_{\bullet-1, q+1}^{1}(X) \longrightarrow 0 .
$$

3. If $f$ is proper, then $\Delta: E_{r, q}^{1}(X)^{(-)} \rightarrow E_{r, q-1}^{1}(Y)$ can be described as

$$
\Delta_{r, q}^{1}: E_{r, q}^{1}(X) \stackrel{s_{j^{*}}}{\longrightarrow} E_{r+1, q-1}^{1}(\mathfrak{X}) \stackrel{d^{1}}{\longrightarrow} E_{r, q-1}^{1}(\mathfrak{X}) \stackrel{s_{i_{*}}}{\longrightarrow} E_{r, q-1}^{1}(Y),
$$

where $s_{i_{*}}$ and $s_{j^{*}}$ are the natural sections of $i_{*}$ and $j^{*}$ given by and projection and inclusion:

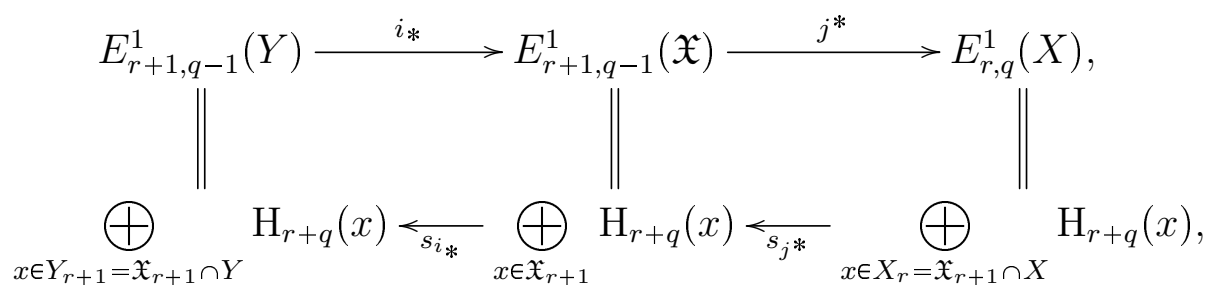

Note, that $s_{i_{*}}$ and $s_{j^{*}}$ may not commute with the differentials $d^{1}: E_{r, q}^{1} \rightarrow$ $E_{r-1, q}^{1}$ and therefore may not be morphisms of complexes.

Proof. $\quad$ 1. For $Z \in \mathcal{Z}_{r}(X)$, let $\bar{Z}$ be the closure in $\mathfrak{X}$. We then have $\operatorname{dim} \bar{Z} \leqslant r+1$ and $\operatorname{dim} \bar{Z} \cap Y \leqslant r$. So $\bar{Z} \cap Y \in \mathcal{Z}_{r}(Y)$ and the morphism of homology theories $\delta: \mathrm{H}^{(X)}[1] \rightarrow \mathrm{H}^{(Y)}$ then gives the spectral sequence including the change of signs.

2. Clear from definition and $\mathfrak{X}_{r} \cap X=X_{r-1}$ and $\mathfrak{X}_{r} \cap Y=Y_{r}$.

3. Clear by 1 .

Corollary 2.3.2. Let the notations be like in 2.3.1 and let $f: \mathfrak{X} \rightarrow S$ be proper. Then for all integers $q$ there is a long exact sequence:

$$
\cdots \longrightarrow E_{r, q}^{2}(Y) \stackrel{i_{*}}{\longrightarrow} E_{r, q}^{2}(\mathfrak{X}) \stackrel{j^{*}}{\longrightarrow} E_{r-1, q+1}^{2}(X) \stackrel{\delta=\Delta}{\longrightarrow} E_{r-1, q}^{2}(Y) \longrightarrow \cdots,
$$

where the maps $\delta$ can be identified with $\Delta$.

Proof. The claim follows from 2.3.1 and the snake lemma. 


\section{4 Étale homology theory over fields}

Here we will treat the special case of étale homology theory over fields $k$ and analyse the support of the first page of its niveau spectral sequence using the cohomological dimension of $k$. Another main point in this section is, that for a projective simple normal crossing variety $Y$ over a finite field we can compute its Kato homology groups out of the homology groups of its dual complex $\Gamma_{Y}$, which was shown in [JS03.

Notation 2.4.1. Let $k$ be a field and let $T$ be a scheme of finite type over $k$. For integers $n, i$ we will define the complex of étale sheaves $\mathbb{Z} / n(i)$ on $T$. We consider three cases:

1. If $n$ is invertible in $k$ and $i$ is arbitrary, we put $\mathbb{Z} / n(i)$ to be the usual $i$-fold Tate twist of the constant sheaf $\mathbb{Z} / n$.

2. If $k$ is a perfect field of $\operatorname{char}(k)=p>0$ and $n=p^{m}$ with an integer $m \geqslant 1$ and $i \geqslant 0$, we define the complex of étale sheaves

$$
\mathbb{Z} / p^{m}(i):=W_{m} \Omega_{T, \log }^{i}[-i]
$$

to be the logarithmic de Rham-Witt sheaf defined in [Ill79].

3. If $k$ is a perfect field of $\operatorname{char}(k)=p>0$ and $n=p^{m} \cdot n^{\prime}$ with $n^{\prime}$ prime to $p$ and an integer $m \geqslant 1, i \geqslant 0$, we put

$$
\mathbb{Z} / n(i):=\mathbb{Z} / n^{\prime}(i) \oplus \mathbb{Z} / p^{m}(i)
$$

Note that $\mathbb{Z} / n(0)$ is just the constant sheaf $\mathbb{Z} / n$ and that $W_{m} \Omega_{k, \log }^{i}$ is not defined for $i<0$ and vanishes for $i>0$.

Definition 2.4.2 (Étale homology theory over fields). Let $k$ be a field and $S=$ $\operatorname{Spec}(k)$ and $n, b$ be fixed integers. We consider only two cases:

1. $n$ is invertible in $k$ and $b$ is arbitrary.

2. If $k$ is a perfect field of $\operatorname{char}(k)=p>0$ and $n=p^{m} \cdot n^{\prime}$ with $n^{\prime}$ prime to $p$ and an integer $m \geqslant 1$ and $b \leqslant 0$.

We then define the étale homology theory on $\operatorname{Sch}_{\mathrm{sft}}(S)$ given by

$\mathrm{H}_{a}(T \mid k, \mathbb{Z} / n(b)):=\mathrm{H}_{a}(T \mid S, \mathbb{Z} / n(b)):=\mathrm{H}_{a}(T \mid S ; \mathbb{Z} / n(-b)):=\mathrm{H}^{-a}\left(T_{e ́ t}, R f^{!} \mathbb{Z} / n(-b)\right)$

with $f: T \rightarrow S$ and where $R f^{!}$is the right adjoint of $R f_{!}$defined in [AGV73] XVIII 3.1.4. For the definition of the complex of étale sheaves $\mathbb{Z} / n(-b)$ on $S$ we use 2.4.1 
(with the restrictions to the numbers $n, b)$.

Note that for a perfect field of $\operatorname{char}(k)=p>0$ we have

$$
\mathrm{H}_{a}\left(T \mid k, \mathbb{Z} / p^{m}(b)\right)=\mathrm{H}^{-a+b}\left(T, R f^{!} W_{m} \Omega_{k, \log }^{-b}\right) .
$$

Futhermore, if $\ell$ is a prime, we put

$$
\mathrm{H}_{a}\left(T \mid k, \mathbb{Q}_{\ell} / \mathbb{Z}_{\ell}(b)\right):=\mathrm{H}_{a}\left(T \mid k, \mathbb{Z} / \ell^{\infty}(b)\right):=\lim _{m \in \mathbb{N}} \mathrm{H}_{a}\left(T \mid k, \mathbb{Z} / \ell^{m}(b)\right) .
$$

Theorem 2.4.3. Let $k$ be a field and $X$ be a scheme separated and of finite type over $k$. Then for the étale homology theory over $k$ and integers $n, b$ like in 2.4.2 we have:

1. There are canonical isomorphisms

$$
\mathrm{H}_{a}(x \mid k, \mathbb{Z} / n(b)) \cong \mathrm{H}^{2 r-a}(\kappa(x), \mathbb{Z} / n(r-b))
$$

for all $x \in X_{r}$. In particular, we have $E_{r, q}^{1}(X \mid k, \mathbb{Z} / n(b))=0$ for all $q>r$, and, trivially, $E_{r, q}^{1}(X \mid k, \mathbb{Z} / n(b))=0$ for all $r<0$ and $r>\operatorname{dim} X$.

2. If the cohomological $l$-dimension $\mathrm{cd}_{l}(k) \leqslant c$ for all primes $l$ dividing $n$, then we have $E_{r, q}^{1}(X \mid k, \mathbb{Z} / n(b))=0$ for all $q<-c$, and, in particular, canonical edge morphisms

$$
\epsilon(X \mid k): \mathrm{H}_{a-c}\left(X \mid k, \mathbb{Z} / n(b) \longrightarrow E_{a,-c}^{2}(X \mid k, \mathbb{Z} / n(b))\right.
$$

So the $E^{1}$-term of the niveau spectral sequence

$$
E_{r, q}^{1}(X \mid k, \mathbb{Z} / n(b))=\bigoplus_{x \in X_{r}} \mathrm{H}^{r-q}(\kappa(x), \mathbb{Z} / n(r-b)) \Longrightarrow \mathrm{H}_{r+q}(X \mid k, \mathbb{Z} / n(b))
$$


looks like

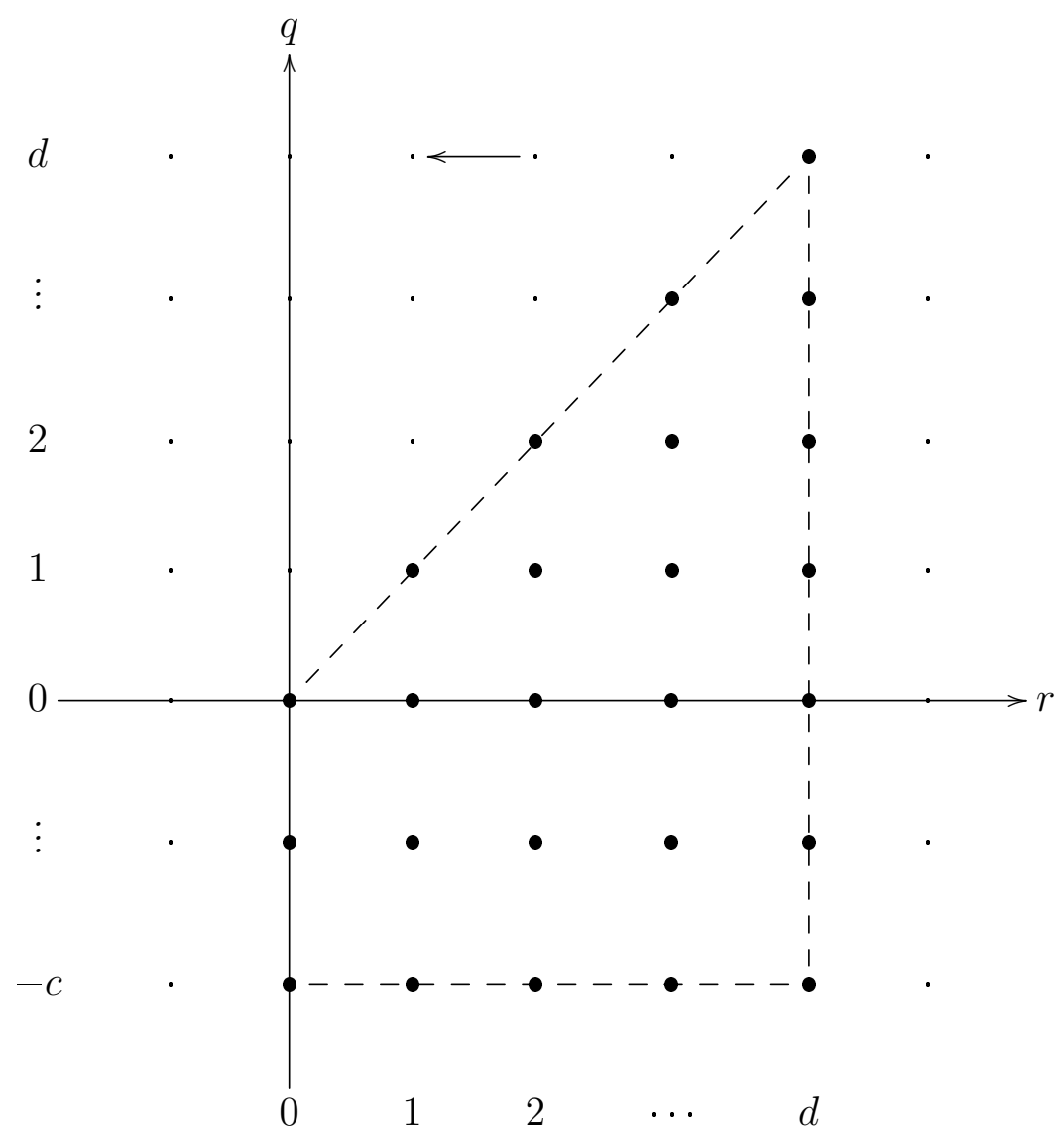

Furthermore, the niveau spectral sequence of the shifted homology theory given by $\mathrm{H}_{\bullet-c}\left({ }_{-} \mid k, \mathbb{Z} / n(b)\right)$ is induced by an exact $\partial$-couple.

3. If $X$ is smooth and of pure dimension $d$ over $k$, then there are canonical and so called purity-isomorphisms

$$
\begin{array}{ccc}
R f ! \mathbb{Z} / n(-b) & \cong & \mathbb{Z} / n(d-b)[2 d], \\
\mathrm{H}_{a}(X \mid k, \mathbb{Z} / n(b)) & \cong & \mathrm{H}^{2 d-a}\left(X_{e ́ t}, \mathbb{Z} / n(d-b)\right) .
\end{array}
$$

Proof. See JS03 Thm. 2.14. The prove uses purity isomorphisms given by the Poincaré duality AGV73] XVIII 3.2.5 in the case $n$ prime to $\operatorname{char}(k)$ and [JSS09] for the other case. For finite fields this can also be deduced from [Mos99] combined with Artin-Verdier duality [AGV73] XVIII 3.1.4 and duality for Galois cohomology of $k$.

Remark 2.4.4. The main property of $R f^{!} \mathbb{Z} / n$ are the purity isomorphisms for smooth $f$ and the follwing purity isomorphism for closed immersions $f$ between regular noetherian schemes. Note that for closed immersions $f$ the functor $R f^{!}$ coincide with the derived functor of $f^{!}$given in [Mil80] Ch. II 3.13. 
Theorem 2.4.5 (Absolute purity theorem by Gabber). Let $f: Z \hookrightarrow W$ be a closed immersion of noetherian regular schemes of pure codimension c, i.e. every irreducible component of $Z$ has codimension $c$ in the corresponding components of $W$. Let $n$ be an integer invertible on $W$, then the cycle class map gives a canonical isomorphism

$$
\mathbb{Z} / n \stackrel{\sim}{\rightarrow} f^{!} \mathbb{Z} / n(c)[2 c]
$$

in $D^{+}\left(Z_{\text {ét }}, \mathbb{Z} / n\right)$, the derived category of complexes of étale $\mathbb{Z} / n$-module sheaves on $Z_{\text {ét }}$ bounded below.

Proof. See [Fuj02 Thm. 2.1.1.

Lemma 2.4.6 (Cohomological descent). Let $Y=\bigcup_{i=1}^{n} Y_{i}$ be a scheme which is covered by a finite number of closed subschemes $Y_{i}$. Let $i^{[r]}: Y^{[r]} \rightarrow Y$ be the canonical map from the disjoint union of the $r$-fold intersections of the $Y_{i}$ to $Y$ for $r \geqslant 0$. Then for every étale sheaf $F$ on $Y$ there is an exact complex

$$
0 \rightarrow F \rightarrow i_{*}^{[0]} i^{[0] *} F \rightarrow i_{*}^{[1]} i^{[1] *} F \rightarrow \cdots \rightarrow i_{*}^{[n-1]} i^{[n-1] *} F \rightarrow 0
$$

where the differential maps are given by the alternating sums of the restriction maps. Furthermore, there is a spectral sequence of cohomological type

$$
{ }^{I} E_{1}^{r, q}=\mathrm{H}^{q}\left(Y^{[r]}, i^{[r] *} F\right) \Longrightarrow \mathrm{H}^{r+q}(Y, F) \text {. }
$$

Proof. Cf. Jan. The spectral sequence is obtained as the hypercohomology spectral sequence, cf. Wei95 Ch. 5, for the naive filtration of the exact complex

$$
i_{*}^{[0]} i^{[0] *} F \rightarrow i_{*}^{[1]} i^{[1] *} F \rightarrow \cdots \rightarrow i_{*}^{[n-1]} i^{[n-1] *} F \rightarrow 0 .
$$

So it suffices to show the exactness of the given complex, which is proven by induction on the number of closed subschemes $n$. For $n=1$ this is trivial. The induction step is achieved by the exact sequence of sheaves

$$
0 \rightarrow F \rightarrow i_{*} i^{*} F \oplus j_{*} j^{*} F \rightarrow k_{*} k^{*} F \rightarrow 0
$$

where $i: \hat{Y} \hookrightarrow Y$ with $\hat{Y}=\bigcup_{i=1}^{n-1} Y_{i}$ and $j: Y_{n} \hookrightarrow Y$ and $k: \hat{Y} \cap Y_{n} \hookrightarrow Y$. The exactness is immediately seen on the stalks.

Example 2.4.7. Let the notations be like in 2.4.6 and let $Y$ be a d-dimensional normal crossing variety over a field $k$ of cohomological n-dimension $c, n$ not divisible by $\operatorname{char}(k)$, and let $F=\mathbb{Z} / n(b)$. Then ${ }^{I} E$ is a first quadrant spectral sequence of 
cohomological type, where the ${ }^{I} E_{1}$-term looks like:

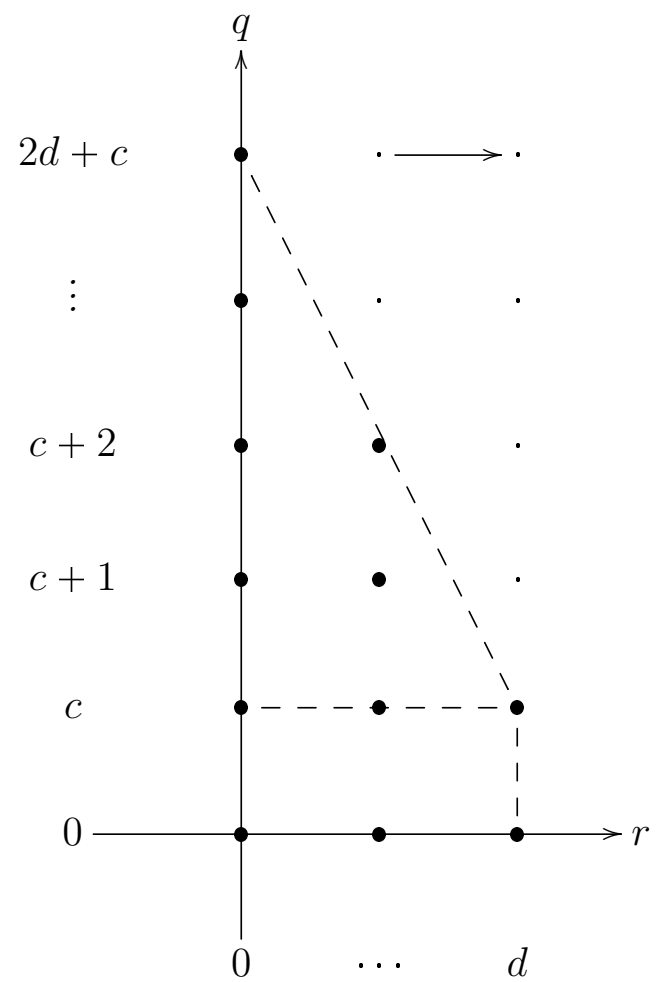

Proof. Since $Y$ is a normal crossing variety, $Y^{[r]}$ is of dimension at most $d-r$ and with a cohomological dimension argument, see [Mil80] Ch. VI $\S 1$ Cor. 1.4, we get the vanishing of the ${ }^{I} E_{1}$-term outside the marked area given above.

Lemma 2.4.8 (Homological descent). Let $Y=\bigcup_{i=1}^{n} Y_{i}$ be a separated scheme of finite type over a field $k$ which is covered by a finite number of closed subschemes $Y_{i}$. Let $Y^{[r]}$ be the disjoint union of the $r$-fold intersections of the $Y_{i}$ for $r \geqslant 0$. Let $n, b$ integers like in 2.4.2. Then there is a spectral sequence of homological type

$$
{ }^{I} E_{r, q}^{1}=\mathrm{H}_{q}\left(Y^{[r]} \mid k, \mathbb{Z} / n(b)\right) \Longrightarrow \mathrm{H}_{r+q}(Y \mid k, \mathbb{Z} / n(b)) .
$$

Proof. Cf. Jan]. This follows from the exact sequence in 2.4.6. Let $I$ be an injective representative of $R f^{!} \mathbb{Z} / n(-b)$, where $f: Y \rightarrow \operatorname{Spec}(k)$ is the structure morphism. Then for any étale sheaf $F$ the complex

$$
\ldots \operatorname{Hom}\left(i_{*}^{[\bullet]_{i}[\bullet] *} F, I\right) \rightarrow \operatorname{Hom}(F, I) \rightarrow 0
$$

is exact by 2.4.6 and the injectivity of $I$. By adjunction

$$
\operatorname{Hom}\left(i_{*}^{[k]} i^{[k] *} F, I\right)=\operatorname{Hom}\left(F, i_{*}^{[k]} R i^{[k] !} I\right),
$$


we get an exact complex

$$
\ldots \operatorname{Hom}\left(F, i_{*}^{[\bullet]} R i^{[\bullet] !} I\right) \rightarrow \operatorname{Hom}(F, I) \rightarrow 0
$$

for any étale sheaf $F$. Therefore the complex

$$
\cdots \rightarrow i_{*}^{[1]} R i^{[1] !} I \rightarrow i_{*}^{[0]} R i^{[0] !} I \rightarrow I \rightarrow 0
$$

is exact. From this we obtain a double complex and an associated spectral sequence like in 2.4.6.

Example 2.4.9. Let the notations be like in 2.4.8 and let $Y$ be a simple normal crossing variety of pure dimension d over a field $k$ of cohomological $n$-dimension $c$, $n$ not divisible by $\operatorname{char}(k)$, and let $F=\mathbb{Z} / n(b)$. Then ${ }^{I} E$ is a first quadrant spectral sequence of homological type, where the ${ }^{I} E_{1}$-term looks like:

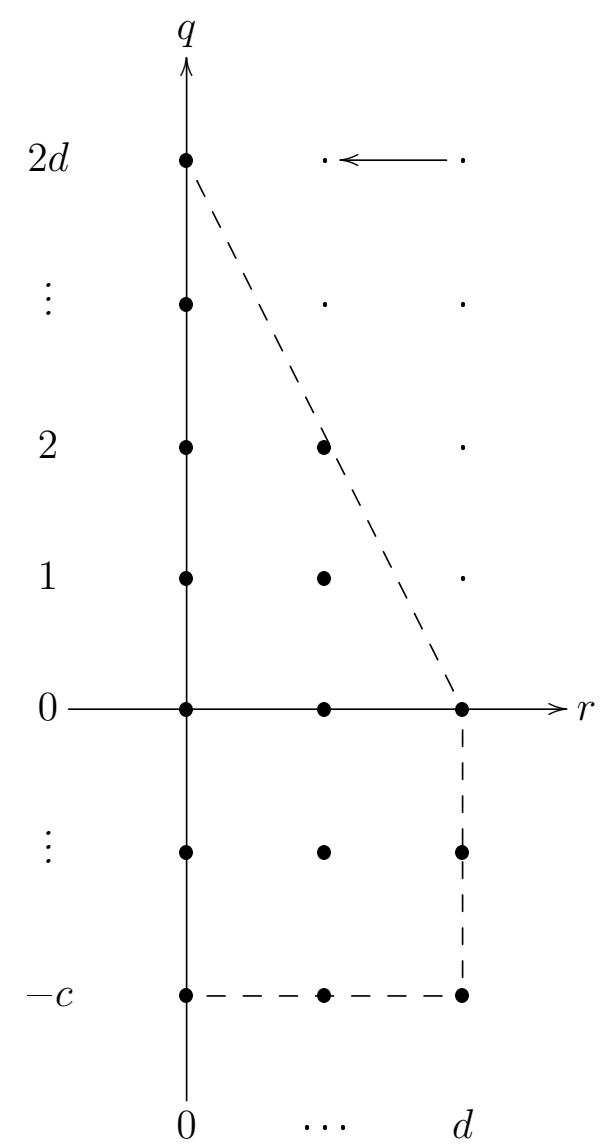

Proof. Purity and a cohomological dimension argument, see Mil80 Ch. VI $\S 1$ Cor. 1.4 , gives the vanishing of the ${ }^{I} E_{1}$-term outside the marked area given above. 
Definition/Lemma 2.4.10 (Kato homology groups). Let the notations be like in 2.4.3, then the edge morphisms $\epsilon(X \mid k)$ induce a morphism of homology theories on $\operatorname{Sch}_{\text {stt }}(\operatorname{Spec}(k))$ :

$$
\epsilon: \mathrm{H}_{\bullet-c}\left({ }_{-} \mid k, \mathbb{Z} / n(b)\right) \longrightarrow E_{\bullet,-c}^{2}\left({ }_{-} \mid k, \mathbb{Z} / n(b)\right)=: \mathrm{H}_{\bullet}^{K}\left({ }_{-} \mid k, \mathbb{Z} / n(b)\right) .
$$

The right homology theory is called the Kato homology theory of the étale homology theory for fields $k$ of cohomological dimension $\operatorname{cd}_{n}(k) \leqslant c$.

Proof. Use 2.2.2. Also see [JS03] Lem. 2.16.

Conjecture 2.4.11 (Kato's conjecture, cf. 2.5.7). Let $Y$ be a regular proper variety over a finite field. Then for every integer $n>0$ there is an isomorphism

$$
\mathrm{H}_{a}^{K}(Y \mid k, \mathbb{Z} / n(0)) \stackrel{\sim}{\rightarrow}\left\{\begin{array}{ccc}
(\mathbb{Z} / n)^{\pi_{0}(Y)} & \text { for } & a=0 \\
0 & \text { for } & a \neq 0
\end{array}\right.
$$

Theorem 2.4.12 (Kerz, Saito). Kato's conjecture 2.4.11 is true if $n>0$ is not divisible by the characteristic $p=\operatorname{char}(k)$.

Proof. See [KS10 Thm. 0.1, 8.1, resp., cf. 2.5.8. The proof uses the Bloch-Katoconjecture 2.4.15.

Theorem 2.4.13. Let $Y$ be a projective simple normal crossing variety of pure dimension d over a finite field $k$ and let $\Gamma_{Y}$ be the dual complex to an ordering of the irreducible components $\left(Y,\left(Y_{i}\right)_{i \in I},(I,<)\right)$. If Kato's conjecture 2.4.11 is true for all $i \leqslant a$, all $Y^{[r]}$ for $r \geqslant 0$ and an arbitrary integer $n>0$, then there is a canonical isomorphism

$$
\mathrm{H}_{a}^{K}(Y \mid k, \mathbb{Z} / n(0)) \stackrel{\sim}{\rightarrow} \mathrm{H}_{a}\left(\Gamma_{Y}, \mathbb{Z} / n\right)
$$

In particular, this isomorphism holds for every integer $n>0$ and $a=0,1,2,3,4$, or if $n$ is not divisible by $p=\operatorname{char}(k)$ and $a$ is arbitrary.

Proof. See [JS03] Thm. 3.9 and Thm. 1.4, resp.. For the special cases $a=3,4$ use [KS10] Thm. 8.1.

Lemma 2.4.14 (Changing the coefficients). Let $\ell$ be an arbitrary prime number, let $k$ be a field of finite cohomological $\ell$-dimension $\operatorname{cd}_{\ell}(k) \leqslant c$, let $V$ be a scheme separated and of finite type over $k$. Then for every $\nu \geqslant 1$ and every $i \geqslant 0$ there is an exact sequence

$\mathrm{H}_{i+1}^{K}\left(V \mid k, \mathbb{Q}_{\ell} / \mathbb{Z}_{\ell}(1-c)\right) / \ell^{\nu} \hookrightarrow \mathrm{H}_{i}^{K}\left(V \mid k, \mathbb{Z} / \ell^{\nu}(1-c)\right) \rightarrow \mathrm{H}_{i}^{K}\left(V \mid k, \mathbb{Q}_{\ell} / \mathbb{Z}_{\ell}(1-c)\right)\left[\ell^{\nu}\right]$. 
Proof. See [JS03] Lem. 7.3 using 2.4.15 and cf. [CT93] §2: Let $\ell \neq \operatorname{char}(k)$. The case $\ell=\operatorname{char}(k)$ follows analogically by using the logarithmic de Rham-Witt sheafs and [BK86] Thm. 2.1. The exact sequence

$$
0 \rightarrow \mathbb{Z} / \ell^{\nu}(r+c-1) \stackrel{\iota}{\rightarrow} \mathbb{Q}_{\ell} / \mathbb{Z}_{\ell}(r+c-1) \stackrel{\cdot \ell^{\nu}}{\rightarrow} \mathbb{Q}_{\ell} / \mathbb{Z}_{\ell}(r+c-1) \rightarrow 0
$$

gives rise to the exact sequence

$$
\begin{gathered}
\mathrm{H}^{r+c-1}\left(\kappa(x), \mathbb{Q}_{\ell} / \mathbb{Z}_{\ell}(r+c-1)\right) \stackrel{\ell^{\nu}}{\longrightarrow} \mathrm{H}^{r+c-1}\left(\kappa(x), \mathbb{Q}_{\ell} / \mathbb{Z}_{\ell}(r+c-1)\right) \stackrel{\delta}{\longrightarrow} \\
\mathrm{H}^{r+c}\left(\kappa(x), \mathbb{Z} / \ell^{\nu}(r+c-1)\right) \stackrel{\iota}{\longrightarrow} \mathrm{H}^{r+c}\left(\kappa(x), \mathbb{Q}_{\ell} / \mathbb{Z}_{\ell}(r+c-1)\right) \stackrel{\cdot \ell^{\nu}}{\longrightarrow} \\
\mathrm{H}^{r+c}\left(\kappa(x), \mathbb{Q}_{\ell} / \mathbb{Z}_{\ell}(r+c-1)\right) \stackrel{\delta}{\longrightarrow} \mathrm{H}^{r+c+1}\left(\kappa(x), \mathbb{Z} / \ell^{\nu}(r+c-1)\right) \longrightarrow,
\end{gathered}
$$

where $x \in V_{r}$ and $\operatorname{cd}_{\ell} \kappa(x) \leqslant c+r$ by [Ser02] Ch.II $\S 4+6$. So the group

$$
\mathrm{H}^{r+c+1}\left(\kappa(x), \mathbb{Z} / \ell^{\nu}(r+c-1)\right)
$$

vanishes by the cohomological dimension. And the first $\ell^{\nu}$-map is surjective by the divisibility of

$$
\mathrm{H}^{r+c-1}\left(\kappa(x), \mathbb{Q}_{\ell} / \mathbb{Z}_{\ell}(r+c-1)\right)
$$

by 2.4.15. So there remains a short exact sequence

$\mathrm{H}^{r+c}\left(\kappa(x), \mathbb{Z} / \ell^{\nu}(r+c-1)\right) \stackrel{\iota}{\hookrightarrow} \mathrm{H}^{r+c}\left(\kappa(x), \mathbb{Q}_{\ell} / \mathbb{Z}_{\ell}(r+c-1)\right) \stackrel{\cdot \ell^{\nu}}{\rightarrow} \mathrm{H}^{r+c}\left(\kappa(x), \mathbb{Q}_{\ell} / \mathbb{Z}_{\ell}(r+c-1)\right)$.

Summing up over all $x \in V_{r}$ and using 2.4 .3 we get the short exact sequence

$0 \rightarrow E_{r,-c}^{1}\left(V \mid k, \mathbb{Z} / \ell^{\nu}(1-c)\right) \stackrel{\iota}{\rightarrow} E_{r,-c}^{1}\left(V \mid k, \mathbb{Q}_{\ell} / \mathbb{Z}_{\ell}(1-c)\right) \stackrel{\cdot \ell^{\nu}}{\rightarrow} E_{r,-c}^{1}\left(V \mid k, \mathbb{Q}_{\ell} / \mathbb{Z}_{\ell}(1-c)\right) \rightarrow 0$,

which is a complex in $r$ by 2.1.6. Taking homology groups gives a long exact sequence

$\cdots \stackrel{\cdot \ell^{\nu}}{\rightarrow} \mathrm{H}_{r+1}^{K}\left(V \mid k, \mathbb{Q}_{\ell} / \mathbb{Z}_{\ell}(1-c)\right) \stackrel{\delta}{\rightarrow} \mathrm{H}_{r}^{K}\left(V \mid k, \mathbb{Z} / \ell^{\nu}(1-c)\right) \stackrel{\iota}{\rightarrow} \mathrm{H}_{r}^{K}\left(V \mid k, \mathbb{Q}_{\ell} / \mathbb{Z}_{\ell}(1-c)\right) \stackrel{\cdot \ell^{\nu}}{\rightarrow} \ldots$

from which follows the assertion.

Theorem 2.4.15 (Bloch-Kato conjecture). For every field L, every prime $\ell \neq$ char $(L)$ and every integer $q \geqslant 0$ the Galois cohomology group

$$
\mathrm{H}^{q}\left(L, \mathbb{Q}_{\ell} / \mathbb{Z}_{\ell}(q)\right)
$$

is divisible.

Proof. The whole proof can be found in the four papers [Voe10b], VVoe10a], [SJ06, [HW09. 


\section{5 Étale homology theory over discrete valuation rings}

Here we will treat the étale homology theory over a discrete valuation ring. The main results in this section is the huge diagram connecting the niveau spectral sequences of the fibres of a scheme over a henselian discrete valuation ring with finite residue field, and the vanishing results concerning Kato's conjecture. From these we will be able to deduce everything about the kernel and cokernel of the reciprocity map in the next chapter.

Definition 2.5.1 (Étale homology theory over discrete valuation rings). Let $S=$ $\operatorname{Spec}(A)$ for a discrete valuation ring $A$ with residue field $k$ and fraction field $K$. Let $j: \eta=\operatorname{Spec}(K) \hookrightarrow S$ be the open immersion of the generic point, and let $i: s=\operatorname{Spec}(k) \hookrightarrow S$ be closed immersion of the special point. Let $n, b$ be fixed integers. We consider the étale homology theory on $\mathrm{Sch}_{\mathrm{sft}}(S)$ given by

$$
\mathrm{H}_{a}(T \mid S, \mathbb{Z} / n(b)):=\mathrm{H}_{a}\left(T \mid S ; \mathbb{Z} / n(-b)_{S}\right):=\mathrm{H}^{-a}\left(T_{e ́ t}, R f^{!} \mathbb{Z} / n(-b)\right)
$$

with $f: T \rightarrow S$. For the definition of the complex of étale sheaves $\mathbb{Z} / n(-b)$ on $S$ we consider two cases:

1. If $n$ is invertible on $S$, and $b$ is arbitrary, we put $\mathbb{Z} / n(-b)$ to be the usual $(-b)$-fold Tate twist of the constant sheaf $\mathbb{Z} / n$.

2. If $\operatorname{char}(K)=0$ and $k$ is a perfect field of $\operatorname{char}(k)=p>0, n=p^{m}$ with an integer $m \geqslant 1$ and $b=-1$, we define the complex of étale sheaves on $S$ by

$$
\mathbb{Z} / p^{m}(1):=\operatorname{Cone}\left(R j_{*}\left(\mathbb{Z} / p^{m}(1)\right)_{\eta} \stackrel{\sigma}{\rightarrow} i_{*}\left(\mathbb{Z} / p^{m}\right)_{s}[-1]\right)[-1]
$$

considered in [JSS09]. Also see [JS03] 2.C using [BBD82] to see, that this is well-defined.

Futhermore, if $\ell$ is a prime, we put

$$
\mathrm{H}_{a}\left(T \mid S, \mathbb{Q}_{\ell} / \mathbb{Z}_{\ell}(b)\right):=\mathrm{H}_{a}\left(T \mid S, \mathbb{Z} / \ell^{\infty}(b)\right):=\lim _{m \in \mathbb{N}} \mathrm{H}_{a}\left(T \mid S, \mathbb{Z} / \ell^{m}(b)\right) .
$$

Lemma 2.5.2. Let $A$ be a henselian discrete valuation ring with finite residue field. Assume that $f: \mathfrak{X} \rightarrow \operatorname{Spec}(A)$ is a regular scheme which is separated and of finite type of pure relative dimension d and with strict semi-stable reduction. Let $n$ be an integer not divisible by the residue characteristic. Then there are canonical isomorphisms

1. $R f^{!} \mathbb{Z} / n \stackrel{\sim}{\rightarrow} \mathbb{Z} / n(d)[2 d]$, 
2. $\mathrm{H}_{a}^{e ́ t}(\mathfrak{X} \mid S, \mathbb{Z} / n(b)) \stackrel{\sim}{\rightarrow} \mathrm{H}_{e ́ t}^{2 d-a}(\mathfrak{X}, \mathbb{Z} / n(d-b))$.

3. $\mathrm{H}_{Y}^{a}(\mathfrak{X}, \mathbb{Z} / n(b)) \cong \mathrm{H}_{2 d-a}^{e ́ t}(Y \mid S, \mathbb{Z} / n(d-b))$ for every closed subscheme $Y \hookrightarrow \mathfrak{X}$.

4. If $f$ is proper, then we have an isomorphism

$$
\mathrm{H}_{a}^{e ́ t}(\mathfrak{X} \mid S, \mathbb{Z} / n(b)) \stackrel{\sim}{\rightarrow} \mathrm{H}_{\text {ét }}^{2 d-a}\left(\mathfrak{X}_{s}, \mathbb{Z} / n(d-b)\right),
$$

where $\mathfrak{X}_{s}$ is the special fibre of $f$.

Proof. The proof is given in Jan]. The third and second isomorphism follows from the first. The first isomorphism is a special case of Grothendiecks's purity conjecture, cf. Gro77 I.5.1 and 2.4.5 from Fuj02, and it is prooven using analogous results from [RZ82]. The last point then is an application of the proper base change theorem.

Proposition 2.5.3. Let the notations be like in 2.5.1.

1. There are isomorphisms of homology theories on $\operatorname{Sch}_{\mathrm{sft}}(\eta)$ and $\mathrm{Sch}_{\mathrm{sft}}(s)$ given by:

$$
\begin{aligned}
\mathrm{H}_{a}\left(\mathfrak{X}_{\eta} \mid S, \mathbb{Z} / n(b)\right) \cong \mathrm{H}_{a}\left(\mathfrak{X}_{\eta} \mid \eta, \mathbb{Z} / n(b)\right), \\
\mathrm{H}_{a}\left(\mathfrak{X}_{s} \mid S, \mathbb{Z} / n(b)\right) \cong \mathrm{H}_{a+2}\left(\mathfrak{X}_{s} \mid s, \mathbb{Z} / n(b+1)\right) .
\end{aligned}
$$

2. There are isomorphisms of spectral sequences

$$
\begin{aligned}
& E_{r, q}^{1}\left(\mathfrak{X}_{\eta} \mid S, \mathbb{Z} / n(b)\right) \cong E_{r, q}^{1}\left(\mathfrak{X}_{\eta} \mid \eta, \mathbb{Z} / n(b)\right), \\
& E_{r, q}^{1}\left(\mathfrak{X}_{s} \mid S, \mathbb{Z} / n(b)\right) \cong E_{r, q+2}^{1}\left(\mathfrak{X}_{s} \mid s, \mathbb{Z} / n(b+1)\right) .
\end{aligned}
$$

3. We trivially have $E_{r, q}^{1}(\mathfrak{X})=0$ for all $r<0$ and $r>\operatorname{dim} \mathfrak{X}$, and analogically for the fibres.

4. If $f: \mathfrak{X} \rightarrow S$ is a proper map, then for all $x \in \mathfrak{X}_{r}$ we have canonical isomorphisms

$$
\mathrm{H}_{a}(x \mid S, \mathbb{Z} / n(b)) \cong \mathrm{H}^{2 r-a-2}(\kappa(x), \mathbb{Z} / n(r-b-1)) .
$$

In particular, we have $E_{r, q}^{1}(\mathfrak{X})=0=E_{r, q}^{1}\left(\mathfrak{X}_{s}\right)$ for all $q>r-2$, and $E_{r, q}^{1}\left(\mathfrak{X}_{\eta}\right)=$ 0 for all $q>r$. The niveau spectral sequence looks like

$$
E_{r, q}^{1}(\mathfrak{X} \mid S, \mathbb{Z} / n(b))=\bigoplus_{x \in \mathfrak{X}_{r}} \mathrm{H}^{r-q-2}(\kappa(x), \mathbb{Z} / n(r-b-1)) \Longrightarrow \mathrm{H}_{r+q}(\mathfrak{X} \mid S, \mathbb{Z} / n(b)) .
$$

Proof. This comes from the purity for discrete valuation rings , cf. [Gro77] I.5.1 and 2.5.2 see [JS03] lemma 2.17 and corollary 2.21. 
Definition 2.5.4 (Kato homology groups). Let the notations be like in 2.5.1 and let $A$ be a henselian discrete valuation ring, $f: \mathfrak{X} \rightarrow S$ be a proper map with special fibre $\mathfrak{X}_{s}=Y$ and generic fibre $\mathfrak{X}_{\eta}=X$. Let $\mathrm{H}=\mathrm{H}\left({ }_{-} \mid S, \mathbb{Z} / n(b)\right)$ be the étale homology theory with $\mathbb{Z} / n(b)$-coefficients. Assume that there is an integer $c \geqslant \sup _{l \mid n} \operatorname{cd}_{l}(k)$. We define the Kato homology groups by:

$$
\begin{aligned}
\mathrm{H}_{a}^{K}(\mathfrak{X} \mid S, \mathbb{Z} / n(b)) & :=E_{a,-c-2}^{2}(\mathfrak{X} \mid S, \mathbb{Z} / n(b)), \\
\mathrm{H}_{a}^{K}(Y \mid S, \mathbb{Z} / n(b)) & :=E_{a,-c-2}^{2}(Y \mid S, \mathbb{Z} / n(b)) \\
& \cong E_{a,-c}^{2}(Y \mid s, \mathbb{Z} / n(b+1))=\mathrm{H}_{a}^{K}(Y \mid s, \mathbb{Z} / n(b+1)), \\
\mathrm{H}_{a}^{K}(X \mid S, \mathbb{Z} / n(b)) & :=E_{a,-c-1}^{2}(X \mid S, \mathbb{Z} / n(b)) \\
& \cong E_{a,-c-1}^{2}(X \mid \eta, \mathbb{Z} / n(b))=\mathrm{H}_{a}^{K}(X \mid \eta, \mathbb{Z} / n(b)) .
\end{aligned}
$$

Remark 2.5.5. Let the assumptions be like in 2.5.4.

- By [Ser02] $\$ 4$ and $\S 6$ we have $\sup _{l \mid n} \operatorname{cd}_{l}(\kappa(x)) \leqslant c+r$ for all $x \in \mathfrak{X}_{r}$.

- By 2.5.3 we then have $E_{r, q}^{1}(\mathfrak{X} \mid S, \mathbb{Z} / n(b))=0=E_{r, q}^{1}(Y \mid S, \mathbb{Z} / n(b))$ for all $q<$ $-c-2$ and $E_{r, q}^{1}(X \mid S, \mathbb{Z} / n(b))=0$ for all $q<-c-1$.

- Recall that there is a long exact sequence by 2.3.2:

$$
\begin{gathered}
0 \longrightarrow \mathrm{H}_{d}^{K}(Y) \stackrel{i_{*}}{\longrightarrow} \mathrm{H}_{d}^{K}(\mathfrak{X}) \stackrel{j^{*}}{\longrightarrow} \mathrm{H}_{d-1}^{K}(X) \stackrel{\Delta}{\longrightarrow} \mathrm{H}_{d-1}^{K}(Y) \longrightarrow \mathrm{H}_{a}^{K}(Y) \stackrel{i_{*}}{\longrightarrow} \mathrm{H}_{a}^{K}(\mathfrak{X}) \stackrel{j^{*}}{\longrightarrow} \mathrm{H}_{a-1}^{K}(X) \stackrel{\Delta}{\longrightarrow} \mathrm{H}_{a-1}^{K}(Y) \stackrel{\longrightarrow}{\longrightarrow} \cdots \\
\cdots \longrightarrow \mathrm{H}_{1}^{K}(\mathfrak{X}) \stackrel{j^{*}}{\longrightarrow} \mathrm{H}_{0}^{K}(X) \stackrel{\Delta}{\longrightarrow} \mathrm{H}_{0}^{K}(Y) \stackrel{i_{*}}{\longrightarrow} \mathrm{H}_{0}^{K}(\mathfrak{X}) \stackrel{\longrightarrow}{\longrightarrow},
\end{gathered}
$$

where $d:=\operatorname{dim} \mathfrak{X}$ and the Kato homology groups are seen over $S$ with coeffcients in $\mathbb{Z} / n(b)$.

- We trivially have $\mathrm{H}_{a}^{K}(Z)=0$ for $a<0$ and $a>\operatorname{dim} Z$.

- There are canonical edge morphisms:

$$
\begin{aligned}
\epsilon: \mathrm{H}_{a-c-2}(\mathfrak{X} \mid S, \mathbb{Z} / n(b)) & \rightarrow \mathrm{H}_{a}^{K}(\mathfrak{X} \mid S, \mathbb{Z} / n(b)), \\
\epsilon: \mathrm{H}_{a-c-2}(Y \mid S, \mathbb{Z} / n(b)) & \rightarrow \mathrm{H}_{a}^{K}(Y \mid S, \mathbb{Z} / n(b)), \\
\epsilon: \mathrm{H}_{a-c-1}(X \mid S, \mathbb{Z} / n(b)) & \rightarrow \mathrm{H}_{a}^{K}(X \mid S, \mathbb{Z} / n(b)) .
\end{aligned}
$$

Proposition 2.5.6. Let $S=\operatorname{Spec}(A)$ be the spectrum of a henselian discrete valuation ring $A$ with fraction field $K$ and residue field $k$, and let $j: \eta=\operatorname{Spec}(K) \hookrightarrow S$ be the open immersion of the generic point, and let $i: s=\operatorname{Spec}(k) \hookrightarrow S$ be closed immersion of the special point. Let $\mathrm{H}=\mathrm{H}\left({ }_{-} \mid S, \mathbb{Z} / n(b)\right)$ be the étale homology theory on $\mathrm{Sch}_{\text {stt }}(S)$ with coefficients in $\mathbb{Z} / n(b)$ for integers $n, b$ like in 2.5.1 such that $\operatorname{cd}_{l}(k) \leqslant c$ for all primes $l$ dividing $n$. Let $f: \mathfrak{X} \rightarrow S$ be a proper map. Then there is a commutative and exact diagram: 


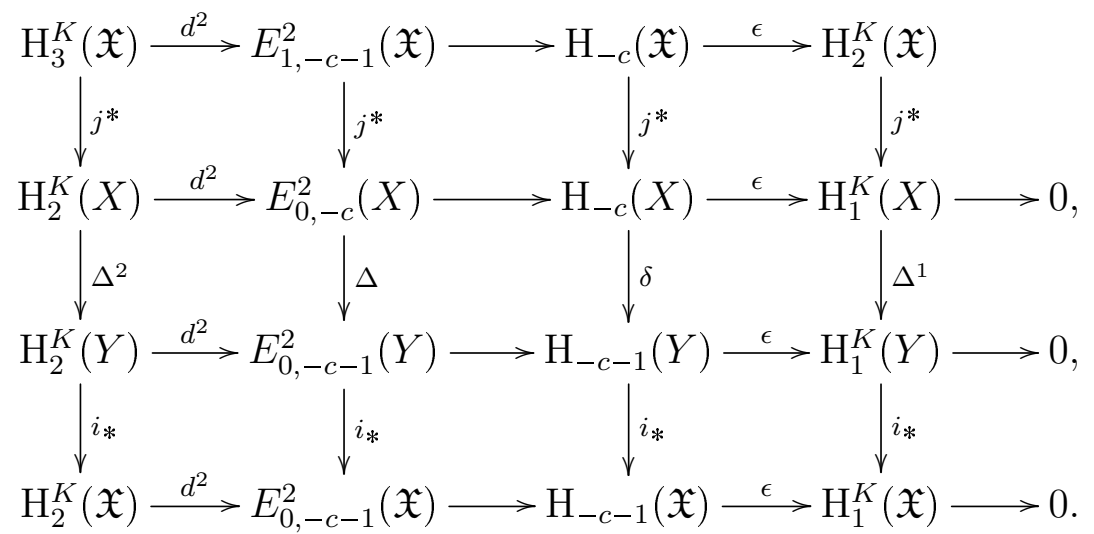

If we can choose $c=1$, then $E_{0,-c}^{2}(Y)=0=E_{0,-c}^{2}(\mathfrak{X})$ and we get a huge commutative and exact diagram:

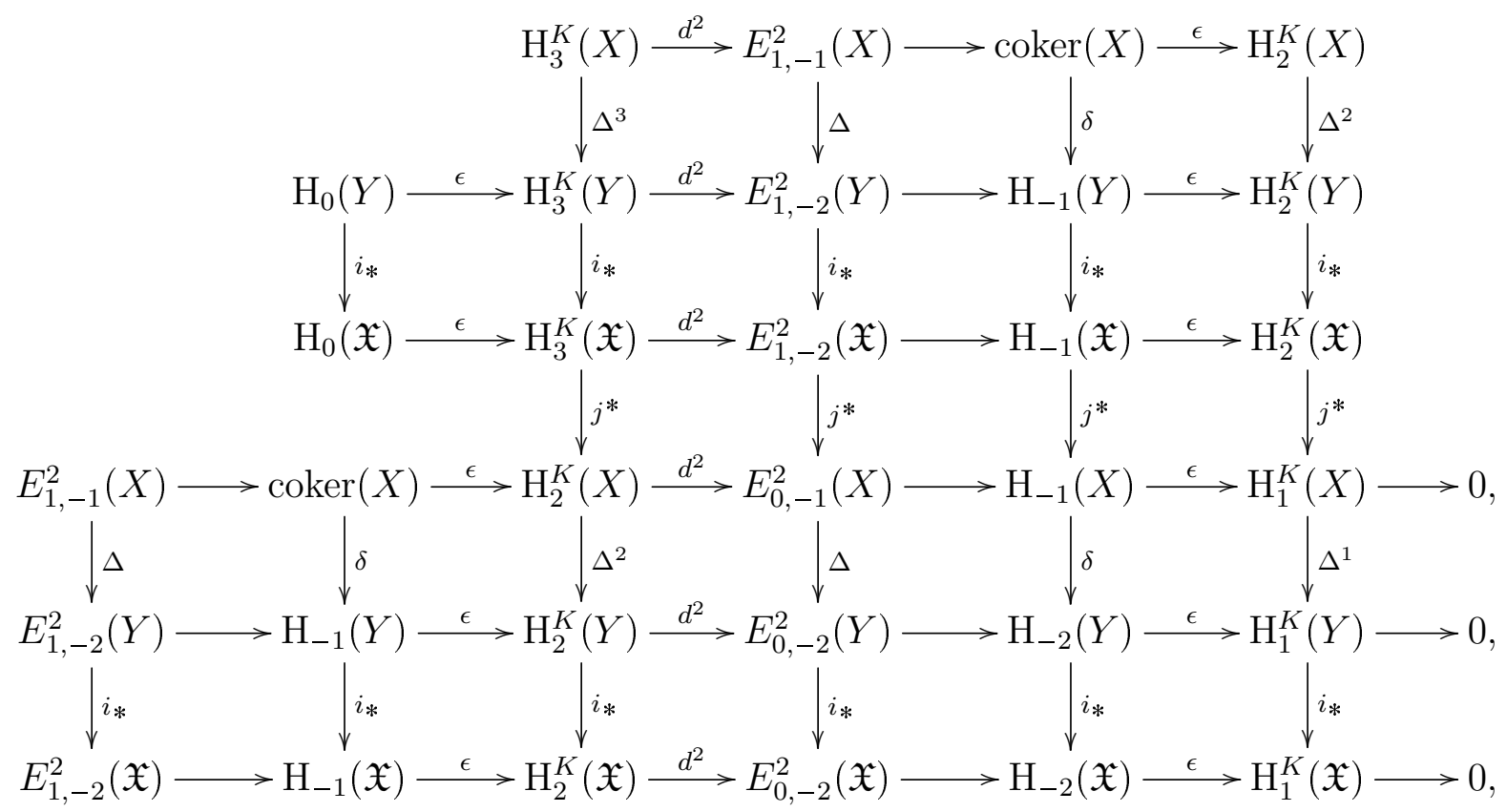

where $\operatorname{coker}(X)=\operatorname{coker}\left(E_{0,0}^{2}(X) \rightarrow \mathrm{H}_{0}(X)\right)$.

Proof. 1. This follows from 2.5.3. The horizontal sequences come from the corresponding 7-term sequences of the niveau spectral sequences. Note that there is a $q$-shift by $-c-2$. Also note for $c=1$ that $E_{0,-c}^{2}(\mathfrak{X})=0$ and therefore $\operatorname{coker}\left(E_{0,-c}^{2}(\mathfrak{X}) \rightarrow \mathrm{H}_{-c}(\mathfrak{X})\right)=\mathrm{H}_{-c}(\mathfrak{X})$ in the line for $\mathfrak{X}$, and also for $Y$.

Conjecture 2.5.7 (Kato's conjecture). Let $\mathfrak{X}$ be a regular proper and flat scheme over a henselian discrete valuation ring $A$ with finite residue field $k$. Then for every integer $n>0$ and $a \geqslant 0$ the group $\mathrm{H}_{a}^{K}(\mathfrak{X} \mid S, \mathbb{Z} / n(-1))$ vanishes, where $S=\operatorname{Spec}(A)$. 
Theorem 2.5.8 (Kerz, Saito). Kato's conjecture 2.5.7 is true if $n$ is not divisible by the residue field characteristic $p=\operatorname{char}(k)$.

Proof. The proof is due to Moritz Kerz and Shuji Saito: KS10 Thm. 8.1 and Thm. 8.4. The proof uses the Bloch-Kato conjecture 2.4.15 and Gabber's refinement of de Jong's alteration in [Ill09].

Lemma 2.5.9. Let the notations be like in 2.5.4 and 2.5.5. Let $\ell \neq \operatorname{char}(K)$ be a prime number. For every integer $i$ there is an exact sequence

$0 \rightarrow \mathrm{H}_{i+1}^{K}\left(\mathfrak{X} \mid S, \mathbb{Q}_{\ell} / \mathbb{Z}_{\ell}(-c)\right) / \ell^{\nu} \rightarrow \mathrm{H}_{i}^{K}\left(\mathfrak{X} \mid S, \mathbb{Z} / \ell^{\nu}(-c)\right) \rightarrow \mathrm{H}_{i}^{K}\left(\mathfrak{X} \mid S, \mathbb{Q}_{\ell} / \mathbb{Z}_{\ell}(-c)\right)\left[\ell^{\nu}\right] \rightarrow 0$.

In particular, if for an integer a the three groups

$$
\mathrm{H}_{a}^{K}\left(\mathfrak{X} \mid S, \mathbb{Q}_{\ell} / \mathbb{Z}_{\ell}(-c)\right)=\mathrm{H}_{a+1}^{K}\left(\mathfrak{X} \mid S, \mathbb{Q}_{\ell} / \mathbb{Z}_{\ell}(-c)\right)=\mathrm{H}_{a+2}^{K}\left(\mathfrak{X} \mid S, \mathbb{Q}_{\ell} / \mathbb{Z}_{\ell}(-c)\right)=0
$$

vanish, then the specialisation map

$$
\mathrm{H}_{a}^{K}\left(X \mid S, \mathbb{Z} / \ell^{\nu}(-c)\right) \rightarrow \mathrm{H}_{a}^{K}\left(Y \mid S, \mathbb{Z} / \ell^{\nu}(-c)\right)
$$

is a natural isomorphism for every $\nu \geqslant 1$.

Proof. This is analogous to 2.4.14. For the second statement in addition use the exact sequence

$$
\mathrm{H}_{a+1}^{K}\left(\mathfrak{X}, \mathbb{Z} / \ell^{\nu}(-c)\right) \rightarrow \mathrm{H}_{a}^{K}\left(X, \mathbb{Z} / \ell^{\nu}(-c)\right) \rightarrow \mathrm{H}_{a}^{K}\left(Y, \mathbb{Z} / \ell^{\nu}(-c)\right) \rightarrow \mathrm{H}_{a}^{K}\left(\mathfrak{X}, \mathbb{Z} / \ell^{\nu}(-c)\right) .
$$

So if $\mathrm{H}_{j}^{K}\left(\mathfrak{X} \mid S, \mathbb{Q}_{\ell} / \mathbb{Z}_{\ell}(-c)\right)$ vanishes for $j=a, a+1, a+2$, then also $\mathrm{H}_{j}^{K}\left(\mathfrak{X} \mid, \mathbb{Z} / \ell^{\nu}(-c)\right)$ vanishes for $j=a, a+1$ and the specialisation map is an isomorphism.

Remark 2.5.10. In low degrees and low dimensions one also is able to state a result, when $K$ is a field of characteristic 0 and where the residue characteristic $\operatorname{char}(k)$ divides $n$ :

Theorem 2.5.11. Let $S=\operatorname{Spec}(A)$ be the spectrum of a henselian discrete valuation ring $A$ with fraction field $K$ and finite residue field $k$, and let $j: \eta=$ $\operatorname{Spec}(K) \hookrightarrow S$ be the open immersion of the generic point, and let $i: s=\operatorname{Spec}(k) \hookrightarrow$ $S$ be closed immersion of the special point. Let $n$ be an integer invertible in $K$ and $\ell$ be a prime different from $\operatorname{char}(K)$, and let $f: \mathfrak{X} \rightarrow S$ be a flat and proper map with smooth generic fibre $X$ and special fibre $Y$. Then we have the following results concerning the Kato homology groups (using $c=1$ and $b=-1$ ) of the étale homology theory on $\operatorname{Sch}_{\mathrm{sft}}(S)$ :

1. $\mathrm{H}_{0}^{K}(\mathfrak{X} \mid S, \mathbb{Z} / n(-1))=0$. 
2. If $X=\mathfrak{X}_{\eta}$ is connected, one has isomorphisms

$$
\Delta_{n}^{0}: \mathrm{H}_{0}^{K}(X \mid S, \mathbb{Z} / n(-1)) \cong \mathrm{H}_{0}^{K}(Y \mid S, \mathbb{Z} / n(-1)) \cong \mathbb{Z} / n .
$$

3. If $\mathfrak{X}$ is regular, then $\mathrm{H}_{1}^{K}(\mathfrak{X}, \mathbb{Z} / n(-1))=0$ and we have an isomorphism

$$
\Delta_{n}^{1}: \mathrm{H}_{1}^{K}(X \mid S, \mathbb{Z} / n(-1)) \cong \mathrm{H}_{1}^{K}(Y \mid S, \mathbb{Z} / n(-1)) .
$$

4. If $f$ is projective and $\mathfrak{X}$ is regular with strict semi-stable reduction, then

$$
\mathrm{H}_{i}^{K}\left(\mathfrak{X} \mid S, \mathbb{Q}_{\ell} / \mathbb{Z}_{\ell}(-1)\right)=0
$$

for $i=0,1,2,3$ and

$$
\Delta_{\ell^{\infty}}^{i}: \mathrm{H}_{i}^{K}\left(X \mid S, \mathbb{Q}_{\ell} / \mathbb{Z}_{\ell}(-1)\right) \rightarrow \mathrm{H}_{i}^{K}\left(Y \mid S, \mathbb{Q}_{\ell} / \mathbb{Z}_{\ell}(-1)\right)
$$

is an isomorphism for $i=0,1,2$, and surjective for $i=3$.

5. If $f$ is projective and $\mathfrak{X}$ is regular with strict semi-stable reduction of relative dimension $d \leqslant 2$, then

$$
\mathrm{H}_{i}^{K}(\mathfrak{X} \mid S, \mathbb{Z} / n(-1))=0
$$

for all $i \geqslant 0$ and

$$
\Delta_{n}^{i}: \mathrm{H}_{i}^{K}(X \mid S, \mathbb{Z} / n(-1)) \cong \mathrm{H}_{i}^{K}(Y \mid S, \mathbb{Z} / n(-1))
$$

is an isomorphism for every $i \geqslant 0$.

Proof. See [JS03 Thm. 1.5, Thm. 1.6. For the last point note that $\mathfrak{X}$ has dimension 3 and therefore $\mathrm{H}_{4}^{K}\left(\mathfrak{X} \mid S, \mathbb{Q}_{\ell} / \mathbb{Z}_{\ell}(-1)\right)$ and all higher groups vanish. So by $2.5 .9 \Delta_{n}^{2}$ is an isomorphism and all higher $\Delta_{n}^{i}$ are the zero-map because of dimension arguments like before. 


\section{Class field theory over local fields}

In this chapter we will deal with class field theory over local fields. The strategy to understand the reciprocity map of a smooth proper variety $X$ over a local field $K$ is to connect it with the reciprocity map of a reduction $Y$ of that variety, which then is given over a finite field $k$ and is understood by the last chapters. To do so, one firstly has to find a sufficiently good model $\mathfrak{X}$ of that variety over the valuation ring $A$ of the given local field $K$. Believing in resolution of singularities one can assume that one has a flat regular model $\mathfrak{X}$ which reduced special fibre $Y$ is a simple normal crossing variety over a finite field $k$. Given such a model one can interpret the reciprocity map as a boundary map in a niveau spectral sequence of a étale homology theory. The variety $X$, the model $\mathfrak{X}$ and its reduction $Y$ then are connected via an excision sequence in that étale homology theory. The recently proven Kato conjecture then states that some of the homology groups of the model vanish. It follows that the kernel and cokernel of the reciprocity map of the variety over a local field then are controlled by the homology groups of the dual complex of its reduction, which by the first chapter also controls the kernel and cokernel of the corresponding reciprocity map of the reduction over the finite residue field. Using this and some other techniques we will see that the kernel of the reciprocity map is the direct sum of a finite group and a $p^{\prime}$-divisible group (i.e. a $\ell$-divisible group, where $\ell$ runs through alls prime numbers diffrent from $p=\operatorname{char}(k))$. Nevertheless one can not assume, that the kernel of the reciprocity map equals the kernel of its reduction. This is because there might be a huge divisible part, which does not occur in the reduction, and some torsion parts coming from higher terms of the niveau spectral sequence.

\subsection{The reciprocity map over local fields modulo $n$}

In this section we will define the reciprocity map for smooth and proper varieties over local fields. Furthermore, we will relate the modulo $n$ version to the niveau spectral sequence associated to the étale homology theory with finite coefficients using the results from the last chapter, duality theorems, some low degree cases of Bloch-Kato's conjecture and the recently proven Kato's conjecture. We follow JS03. 
Definition 3.1.1 (SK-groups). Let $X$ be a noetherian scheme. For every integer $j \geqslant 0$ we put

$$
X_{j}:=\{x \in X \mid \operatorname{dim}(\overline{\{x\}})=j\}
$$

as the set of $j$-dimensional points of $X$. Furthermore, we define the SK-groups to be

$$
\mathrm{SK}_{j}(X):=\operatorname{coker}\left(\bigoplus_{y \in X_{j+1}} K_{j+1}(\kappa(y)) \longrightarrow \bigoplus_{x \in X_{j}} K_{j}(\kappa(x))\right)
$$

Note that the components of the map are given by the boundary maps in algebraic $K$-theory. Also note, that $\mathrm{SK}_{0}(X)=\mathrm{CH}_{0}(X)$.

Definition 3.1.2 (The reciprocity map). Let $K$ be a henselian discrete valuation field with finite residue field and $X$ be a scheme of finite type over $K$. For every $x \in X_{0}$ we then have that $\kappa(x)$ is also a henselian discrete valuation field with finite residue field. So there is a map

$$
\kappa(x)^{\times} \rightarrow G_{\kappa(x)}^{\mathrm{ab}}=\pi_{1}^{\mathrm{ab}}(\kappa(x)) \rightarrow \pi_{1}^{\mathrm{ab}}(X),
$$

where the first map is the 0-dimensional reciprocity map for $\kappa(x)$ and the second is the covariant push-forward map for $\pi_{1}^{\mathrm{ab}}$ of $\operatorname{Spec}(\kappa(x)) \rightarrow X$. Summing all these maps up, we get a map

$$
\rho^{\prime}: \bigoplus_{x \in X_{0}} \kappa(x)^{\times} \rightarrow \pi_{1}^{\mathrm{ab}}(X)
$$

Now, if $X$ is proper over $K$, the map $\rho^{\prime}$ factors through

$$
\rho^{X}: \operatorname{SK}_{1}(X) \longrightarrow \pi_{1}^{\mathrm{ab}}(X)
$$

which is called the reciprocity map of $X$.

Proof. This is due to [Blo81], [KS85], SSai85a] and [Sai85b].

Proposition 3.1.3 (Duality theorems). 1. Let $K$ be a henselian discrete valuation field with finite residue field $k$. Let $f_{\eta}: X \rightarrow \operatorname{Spec}(K)$ be a proper scheme. For any complex $C$ of étale $\mathbb{Z} / n$-module sheaves on $X$ with bounded constructible cohomology sheaves, the pairing

$$
\mathrm{H}^{i}\left(X, D_{X}(C)\right) \times \mathrm{H}^{2-i}(X, C) \rightarrow \mathrm{H}^{2}\left(X, R f_{\eta}^{!} \mathbb{Z} / n(1)\right) \stackrel{\operatorname{tr}_{X}}{\rightarrow} \mathrm{H}^{2}(K, \mathbb{Z} / n(1)) \stackrel{\operatorname{tr}_{\eta}}{\cong} \mathbb{Z} / n
$$

is a perfect pairing of finite groups. Here $D_{X}(C)=R \mathcal{H}$ om $\left(C, R f_{\eta}^{!} \mathbb{Z} / n(1)\right)$, and $\operatorname{tr}_{X}$ is induced by the trace morphisms $R f_{\eta *} R f_{\eta}^{!} \mathbb{Z} / n(1) \rightarrow \mathbb{Z} / n(1)$. 
2. Let $k$ be finite field and $f_{s}: Y \rightarrow \operatorname{Spec}(k)$ be a proper scheme. For any complex $C$ of étale $\mathbb{Z} / n$-module sheaves on $Y$ with bounded constructible cohomology sheaves, the pairing

$$
\mathrm{H}^{i}\left(Y, D_{Y}(C)\right) \times \mathrm{H}^{1-i}(Y, C) \rightarrow \mathrm{H}^{1}\left(Y, R f_{s}^{!} \mathbb{Z} / n\right) \stackrel{\operatorname{tr}_{Y}}{\rightarrow} \mathrm{H}^{1}(k, \mathbb{Z} / n) \stackrel{\operatorname{tr}_{s}}{\cong} \mathbb{Z} / n
$$

is a perfect pairing of finite groups. Here $D_{Y}(C)=R \mathcal{H}$ om $\left(C, R f_{s}^{!} \mathbb{Z} / n\right)$, and $\operatorname{tr}_{Y}$ is induced by the trace morphisms $R f_{s *} R f_{s}^{!} \mathbb{Z} / n \rightarrow \mathbb{Z} / n$.

Proof. See [JS03 Lem. 5.3. This follows from Artin-Verdier duality for $f_{\eta}$ and $f_{s}$ together with the duality theorems for Galois cohomology of $K$ and $k$, cf. SSai98] and CTSS83.

Theorem 3.1.4. Let $S=\operatorname{Spec}(A)$ be the spectrum of a henselian discrete valuation ring $A$ with quotient field $K$ and finite residue field $k$. Let $f: \mathfrak{X} \rightarrow S$ be a regular, proper, flat scheme over $S$ with smooth and connected generic fiber $X$ and strict semi-stable reduction $Y$ as the special fiber. Let $Y=\bigcup_{v \in I} Y_{v}$ be a fixed ordering of the irreducible components of $Y$ and let $\Gamma_{Y}$ be the dual complex to $\left(Y,\left(Y_{i}\right)_{i \in I},(I,<)\right)$. Furthermore, let $n$ either be an integer not divided by $p:=\operatorname{char}(k)$, or let $\operatorname{char}(K)=$ 0 and $p$ divide $n$ then assume that the specialisation map

$$
\mathrm{H}_{2}^{K}(X \mid S, \mathbb{Z} / n(-1)) \cong \mathrm{H}_{2}^{K}(Y \mid S, \mathbb{Z} / n(-1))
$$

is a natural isomorphism, which by 2.5.9 and 2.5.11 is the case if $f$ is projective and

$$
\mathrm{H}_{4}^{K}\left(\mathfrak{X} \mid S, \mathbb{Q}_{p} / \mathbb{Z}_{p}(-1)\right) \quad \text { is p-divisible. }
$$

Then the reciprocity map modulo $n$ fits into a commutative diagram of exact sequences of finite groups

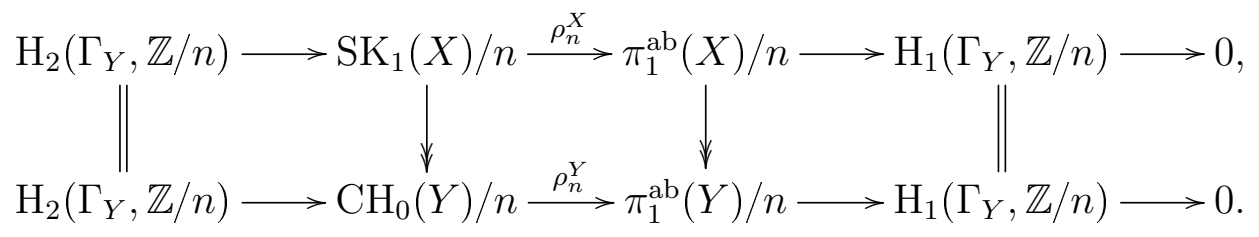

Proof. In 2.5.6 we can use $c=1=\operatorname{cd}(k), b=-1$ and $\mathbb{Z} / n(-1)$-coefficients for 
$\mathrm{H}_{a}\left({ }_{-}\right)=\mathrm{H}_{a}\left({ }_{-} \mid S, \mathbb{Z} / n(-1)\right)$ to get a commutative diagram of exact sequences:

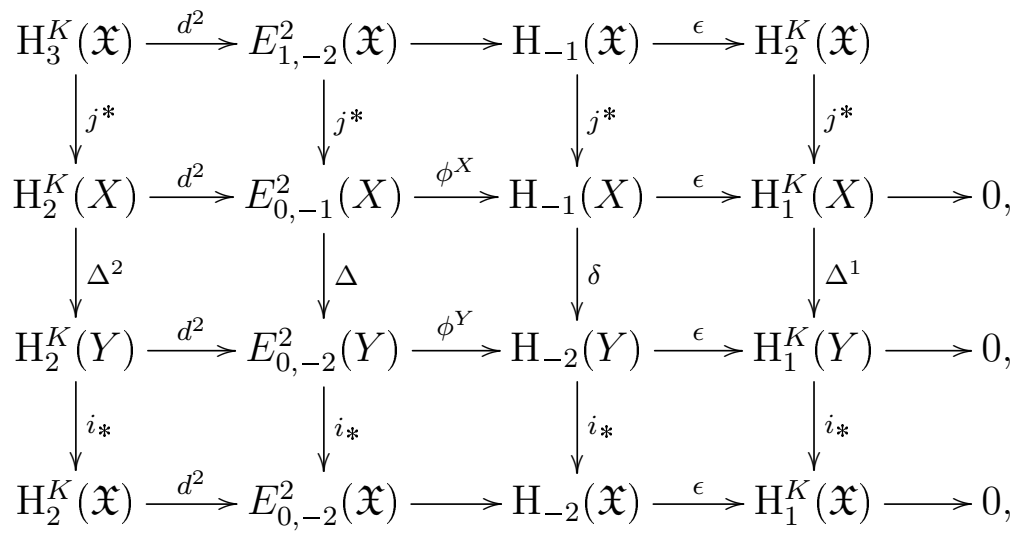

where the groups $\mathrm{H}_{i}^{K}(\mathfrak{X} \mid S, \mathbb{Z} / n(-1))$ vanish for $i=1,2,3$ by 2.5.8, if $n$ is not divided by $\operatorname{char}(K)$ or $p=\operatorname{char}(k)$. So with the assumptions on $p, 2.5 .11$ and 2.4.13 we have natural isomorphisms

$$
\mathrm{H}_{i}^{K}(X \mid S, \mathbb{Z} / n(-1)) \cong \mathrm{H}_{i}^{K}(Y \mid S, \mathbb{Z} / n(-1)) \cong \mathrm{H}_{i}\left(\Gamma_{Y}, \mathbb{Z} / n\right)
$$

for $i=1,2$. And the last group is clearly finite.

By 3.1 .3 and 2.5.3 we have canonical identifications

$$
\begin{aligned}
& \mathrm{H}_{-1}(X \mid S, \mathbb{Z} / n(-1)) \cong \mathrm{H}_{-1}(X \mid \eta, \mathbb{Z} / n(-1))=\mathrm{H}^{1}\left(X, R f_{\eta}^{!} \mathbb{Z} / n(1)\right)
\end{aligned}
$$

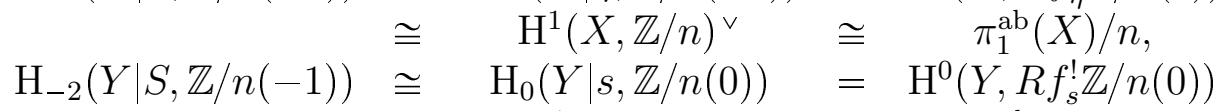

$$
\begin{aligned}
& \cong \mathrm{H}^{1}(Y, \mathbb{Z} / n)^{\vee} \cong \pi_{1}^{\mathrm{ab}}(Y) / n
\end{aligned}
$$

where we can identify $\delta$ with the specialisation map on fundamental groups, which is surjective since $\mathfrak{X}$ is a normal scheme.

The Galois symbol maps $K_{i}(\kappa) / n \rightarrow \mathrm{H}^{i}(\kappa, \mathbb{Z} / n(i))$ in degree $i \leqslant 2$ are isomorphisms by Kummer theory and MS83 and commute with the localisation maps, cf. Kat86] Lem. 1.4. So by 2.5.3 and by the properness of $f: \mathfrak{X} \rightarrow S$ we get indentifications

$$
\begin{aligned}
& E_{0,-1}^{2}(X \mid S, \mathbb{Z} / n(-1)) \\
& =\operatorname{coker}\left(E_{1,-1}^{1}(X \mid S, \mathbb{Z} / n(-1)) \quad \rightarrow \quad E_{0,-1}^{1}(X \mid S, \mathbb{Z} / n(-1))\right) \\
& =\operatorname{coker}\left(\underset{x \in X_{1}=\mathfrak{X}_{2} \cap X}{\bigoplus_{0}} \mathrm{H}_{0}(x \mid S, \mathbb{Z} / n(-1)) \rightarrow \underset{x \in X_{0}=\mathfrak{X}_{1} \cap X}{\bigoplus} \mathrm{H}_{-1}(x \mid S, \mathbb{Z} / n(-1))\right) \\
& \cong \operatorname{coker}\left(\bigoplus_{x \in X_{1}} \mathrm{H}^{2}(\kappa(x), \mathbb{Z} / n(2)) \quad \rightarrow \bigoplus_{x \in X_{0}} \mathrm{H}^{1}(\kappa(x), \mathbb{Z} / n(1))\right) \\
& \cong \operatorname{coker}\left(\bigoplus_{x \in X_{1}} K_{2}(\kappa(x)) / n \quad \rightarrow \bigoplus_{x \in X_{0}} K_{1}(\kappa(x)) / n\right) \\
& =\mathrm{SK}_{1}(X) / n \text {, }
\end{aligned}
$$


and

$$
\begin{aligned}
& E_{0,-2}^{2}(Y \mid S, \mathbb{Z} / n(-1)) \\
& =\operatorname{coker}\left(E_{1,-2}^{1}(Y \mid S, \mathbb{Z} / n(-1)) \rightarrow E_{0,-2}^{1}(Y \mid S, \mathbb{Z} / n(-1))\right) \\
& =\operatorname{coker}\left(\bigoplus_{x \in Y_{1}} \mathrm{H}_{-1}(x \mid S, \mathbb{Z} / n(-1)) \rightarrow \bigoplus_{x \in Y_{0}} \mathrm{H}_{-2}(x \mid S, \mathbb{Z} / n(-1))\right) \\
& \cong \operatorname{coker}\left(\bigoplus_{x \in Y_{1}} \mathrm{H}^{1}(\kappa(x), \mathbb{Z} / n(1)) \rightarrow \bigoplus_{x \in Y_{0}} \mathrm{H}^{0}(\kappa(x), \mathbb{Z} / n(0))\right) \\
& \cong \operatorname{coker}\left(\bigoplus_{x \in Y_{1}} K_{1}(\kappa(x)) / n \quad \rightarrow \bigoplus_{x \in Y_{0}} K_{0}(\kappa(x)) / n\right) \\
& =\mathrm{CH}_{0}(Y) / n \text {, }
\end{aligned}
$$

where the horizontal maps $\phi^{X}$ and $\phi^{Y}$ can be identified with the reciprocity maps $\rho_{n}^{X}$ and $\rho_{n}^{Y}$ given by local duality up to sign, which is shown in [JS03] and [JSS09], respectively. So we get the stated commutative exact diagram of finite groups.

Lemma 3.1.5. Let

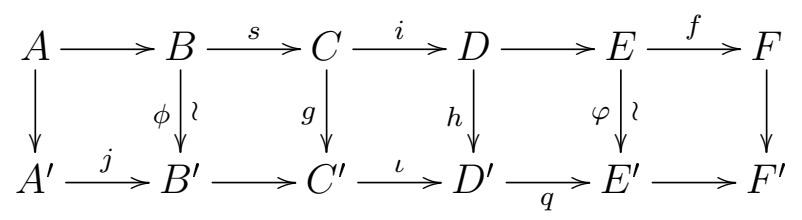

be a commutative diagram of exact sequences, where $\phi$ and $\varphi$ are isomorphisms. Then the sequence

$$
A^{\prime} \stackrel{s \phi^{-1} j}{\longrightarrow} C \stackrel{g \oplus i}{\longrightarrow} C^{\prime} \oplus D \stackrel{\iota-h}{\longrightarrow} D^{\prime} \stackrel{q \varphi^{-1} f}{\longrightarrow} F
$$

is exact.

Proof. Easy diagram chasing.

Remark 3.1.6. If one wants to know in 3.1.4 if the kernels of the reciprocity maps modulo $n$ coincide, one has to write down two more homology groups on the left from the huge diagram 2.5.6 and use 3.1.5. Then we get the following statement:

$$
\operatorname{ker} \rho_{n}^{X} \cong \operatorname{ker} \rho_{n}^{Y} \quad \text { if and only if }
$$

the natural map

$$
E_{1,-2}^{2}(Y \mid S, \mathbb{Z} / n(-1)) \oplus \mathrm{H}_{0}(X \mid S, \mathbb{Z} / n(-1)) \longrightarrow \mathrm{H}_{-1}(Y \mid S, \mathbb{Z} / n(-1))
$$

is surjective. 
Proof. By 3.1.4 $\Delta: \operatorname{ker} \rho_{n}^{X} \rightarrow \operatorname{ker} \rho_{n}^{Y}$ is surjective, and

$$
\operatorname{ker} \Delta=\operatorname{ker}\left(\mathrm{SK}_{1}(X) / n \stackrel{\mathrm{sp} \oplus \rho_{n}^{X}}{\longrightarrow} \mathrm{CH}_{0}(Y) / n \oplus \pi_{1}^{\mathrm{ab}}(X) / n\right) .
$$

And using 3.1.5 applied to the huge diagram 2.5.6 we get the exact sequence

$$
E_{1,-2}^{2}(Y) \oplus \mathrm{H}_{0}(X) \rightarrow \mathrm{H}_{-1}(Y) \rightarrow \mathrm{SK}_{1}(X) / n \rightarrow \mathrm{CH}_{0}(Y) / n \oplus \pi_{1}^{\mathrm{ab}}(X) / n .
$$

So the equivalence follows.

Remark 3.1.7. For surfaces $X$ which have a proper and surjective map to a smooth geometrically connected curve with a smooth conic as generic fibre in [Sza99] the injectivity of $\rho_{n}^{X}$ is shown.

\subsection{Divisibility yoga and completeness meditations}

In this section we will collect all lemmata and auxiliary results we need concerning divisibility questions and completing abelian groups. This will be helpful to transfer results with finite coefficients to analoge results with $\mathbb{Z}_{\ell}-$ or even $\mathbb{Z}$-coefficients.

Notation 3.2.1. For an abelian group $A$ and a set of primes $\mathbb{L}$ let $\mathbb{N}(\mathbb{L})$ be the monoid of all natural numbers which have prime divisiors only in $\mathbb{L}$. We define $A_{\mathbb{L}}$ to be the $\mathbb{L}$-completion

$$
A_{\mathbb{L}}:=\lim _{n \in \mathbb{N}(\mathbb{L})} A / n
$$

and $\hat{A}$ to be

$$
\hat{A}:=\lim _{\substack{n \in \mathbb{N}}} A / n
$$

the $\hat{\mathbb{Z}}$-completion.

Remark 3.2.2. If $B$ is an abelian pro-finite group we have $\hat{B}=B$. If moreover $B$ is a finitely generated pro-finite group we know that $B_{\mathbb{L}}$ is the maximal pro- $\mathbb{L}$ quotient of $B$.

Notation 3.2.3. For an abelian group $A$ and an integer $n$ we denote by ${ }_{n} A, n A$ and $A / n$ the kernel, the image and the cokernel of the multiplication map

$$
A \stackrel{\cdot n}{\longrightarrow} A,
$$

and by

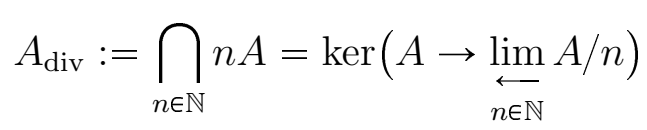


the subgroup of all divisible elements of $A$, and by

$$
A_{\text {Div }}:=\operatorname{im}(\operatorname{Hom}(\mathbb{Q}, A) \rightarrow \operatorname{Hom}(\mathbb{Z}, A) \cong A)
$$

the maximal divisible subgroup of $A$. And we put

$$
A_{\text {tors }}:=\bigcup_{n \in \mathbb{N}}{ }_{n} A=\operatorname{Tor}_{1}^{\mathbb{Z}}(A, \mathbb{Q} / \mathbb{Z})
$$

to be the torsion subgroup of $A$.

Notation 3.2.4. Furthermore, let $A$ be an abelian group and $\mathbb{L}$ be a set of prime numbers. We put $\mathbb{N}(\mathbb{L})$ to be the multiplicative set of all natural numbers with prime divisors only in $\mathbb{L} . \mathbb{N}(\mathbb{L})$ is directedly ordered by divisibility relations. We put

$$
A_{\mathbb{L}-\text { div }}:=\bigcap_{n \in \mathbb{N}(\mathbb{L})} n A=\operatorname{ker}\left(A \rightarrow \lim _{n \in \mathbb{N}(\mathbb{L})} A / n\right)
$$

to be the subgroup of all divisible elements of $A$, and by $A_{\mathbb{L} \text {-Div }}$ the maximal divisible subgroup of $A$ and we put $A_{\mathbb{L} \text {-tors }}$ to be the subgroup of all torsion element that have order in $\mathbb{N}(\mathbb{L})$. If $\mathbb{L}$ is the set of all primes, we then have $A_{\mathbb{L}-\mathrm{div}}=A_{\mathrm{div}}$ and $A_{\mathbb{L} \text {-Div }}=A_{\text {Div }}$ and $A_{\mathbb{L} \text {-tors }}=A_{\text {tors }}$.

Definition 3.2.5 (Mittag-Leffler condition). A projective system $\left(A_{i}, f_{i j}\right)_{i, j \in I}$ of abelian groups fulfills the Mittag-Leffler condition if for each index $i \in I$ there is an index $j \geqslant i$ such that for every $k \geqslant j$ the transition maps $f_{j i}$ and $f_{k i}$ have the same image in $A_{i}$.

And it satisfies the trivial Mittag-Leffler condition if for each index $i \in I$ there is an index $j \geqslant i$ such that the transition map $f_{j i}$ is the zero map.

Lemma 3.2.6. Let $\left(A_{i}, f_{i j}\right)_{i, j \in \mathbb{N}(\mathbb{L})}$ be a projective system of abelian groups satisfying the Mittag-Leffler condition, then for the right derived functor $\lim _{\longleftarrow}^{1}$ of the projective limit we have

$$
\underset{n \in \mathbb{N}(\mathbb{L})}{\lim ^{1}} A_{n}=0 .
$$

If the trivial Mittag-Leffler condition is satisfied, then we also get

$$
\lim _{n \in \mathbb{N}(\mathbb{L})} A_{n}=0
$$

Proof. See [Wei95] Prp. 3.5.7 and notice that the projective system is equivalent to a tower, e.g. to a subsystem of $B_{n}:=A_{n !}$, which then also satisfies the MittagLeffler condition. 
Example 3.2.7. The Mittag-Leffler condition is fulfilled for the index set $\mathbb{N}(\mathbb{L})$ in the following cases:

- All transition maps are the zero-maps.

- The trival Mittag-Leffler condition is satisfied.

- All transition maps are onto.

- All abelian groups $A_{n}$ of the system are finite and the transition maps are arbitrary.

Proof. See [Wei95] Def. 3.5.6 and Ex. 3.5.2.

Lemma 3.2.8. For an abelian group, we always have

$$
A_{\mathbb{L}-\mathrm{Div}} \subseteq A_{\mathbb{L} \text {-div }},
$$

but not necessarily equality. And equality holds, if the projective system $\left\{{ }_{n m} A \stackrel{\cdot m}{\rightarrow}\right.$ $\left.{ }_{n} A\right\}_{n, m \in \mathbb{N}(\mathbb{L})}$ satisfies the Mittag-Leffler condition, e.g. if the groups ${ }_{n} A$ are finite for all $n \in \mathbb{N}(\mathbb{L})$.

Proof. See [Jan88] $\$ 4$ or Wei95] Prop. 3.5.7.

Lemma 3.2.9. Let $\mathbb{L}$ be a set of primes and let $A$ be an abelian group and $\iota: A \rightarrow$ $A_{\mathbb{L}}$ the natural map. Assume one of the following conditions:

- Let $g: A_{\mathbb{L}} \rightarrow B$ be a homomorphism of abelian groups such that there are integers $m, m^{\prime} \in \mathbb{N}(\mathbb{L})$ with $m \cdot B_{\mathbb{L} \text {-tors }}=0=m^{\prime} \cdot \operatorname{ker} g$.

- Let $f: A \rightarrow B$ be a homomorphism of abelian groups such that $A_{\mathbb{L} \text {-div }} \subseteq$ ker $f$ and there are integers $m, m^{\prime} \in \mathbb{N}(\mathbb{L})$ with $m \cdot B_{\mathbb{L} \text {-tors }}=0=m^{\prime} \cdot\left(\operatorname{ker} f / A_{\mathbb{L} \text {-div }}\right)$.

Then we have the equality $A_{\mathbb{L} \text {-Div }}=A_{\mathbb{L} \text {-div }}$.

Proof. Let $n \in \mathbb{N}(\mathbb{L})$ be an arbitrary integer and let $x \in A_{\mathbb{L} \text {-div }}$. Then $n m m^{\prime} \in \mathbb{N}(\mathbb{L})$ and there is a $y \in A$ with $x=n m m^{\prime} y$. We now want to show that $m m^{\prime} y \in A_{\mathbb{L} \text {-div }}$. Then $A_{\mathbb{L} \text {-div }}$ will be $\mathbb{L}$-divisible. Let $(*)$ be the assumption on $m$ and $m^{\prime}$ from above.

- Because of $x \in A_{\mathbb{L} \text {-div }}=\operatorname{ker} \iota$ and the assumptions (*), we have

$$
\begin{aligned}
& 0=g \iota(x)=n m m^{\prime} \cdot g \iota(y), \\
& \Longrightarrow \quad g \iota(y) \quad \in \quad B_{\mathbb{L} \text {-tors }} \\
& \stackrel{*}{\Longrightarrow} 0=m \cdot g \iota(y)=g \iota(m y) \text {, } \\
& \Longrightarrow \quad \iota(m y) \quad \in \quad \operatorname{ker} g \text {, } \\
& \stackrel{*}{\Longrightarrow} 0=m^{\prime} \cdot \iota(m y)=\iota\left(m m^{\prime} y\right) \\
& \Longrightarrow \quad m m^{\prime} y \quad \in \quad \operatorname{ker} \iota \quad=A_{\mathbb{L} \text {-div }} \text {. }
\end{aligned}
$$


- Because of $x \in A_{\mathbb{L} \text {-div }} \subseteq \operatorname{ker} f$ and the assumptions $(*)$, we have

$$
\begin{aligned}
& 0=f(x)=n m m^{\prime} f(y), \\
& \Longrightarrow \quad f(y) \quad \in \quad B_{\mathbb{L} \text {-tors }} \text {, } \\
& \stackrel{*}{\Longrightarrow} 0=m \cdot f(y)=f(m y) \text {, } \\
& \Longrightarrow \quad m y \quad \in \quad \operatorname{ker} f \text {, } \\
& \stackrel{*}{\Longrightarrow} \overline{0}=m^{\prime} \cdot \overline{m y} \in \operatorname{ker} f / A_{\mathbb{L} \text {-div }}, \\
& \Longrightarrow \quad m m^{\prime} y \quad \in \quad A_{\mathbb{L} \text {-div }} \text {. }
\end{aligned}
$$

Lemma 3.2.10. Let $A, B, C$ be abelian groups and $\mathbb{L}$ be a set of prime numbers.

1. If $B \rightarrow C$ is a surjection, we always have a surjection $B_{\mathbb{L}} \rightarrow C_{\mathbb{L}}$.

2. If $A / n$ is finite for every $n \in \mathbb{N}(\mathbb{L})$ or if $\left({ }_{n m} C \stackrel{\cdot m}{\rightarrow}{ }_{n} C\right)_{n, m \in \mathbb{N}(\mathbb{L})}$ satisfies the Mittag-Leffler condition, e.g. if the ${ }_{n} C$ are all finite, then the exact sequence

$$
A \rightarrow B \rightarrow C \rightarrow 0
$$

induces the exact sequence

$$
A_{\mathbb{L}} \rightarrow B_{\mathbb{L}} \rightarrow C_{\mathbb{L}} \rightarrow 0
$$

3. If $\left({ }_{n m} C \stackrel{\cdot m}{\rightarrow}{ }_{n} C\right)_{n, m \in \mathbb{N}(\mathbb{L})}$ satisfies the trivial Mittag-Leffler condition, e.g. if $C_{\mathbb{L} \text {-tors }}$ is of finite exponent, then the exact sequence

$$
0 \rightarrow A \rightarrow B \rightarrow C \rightarrow 0
$$

induces the exact sequence

$$
0 \rightarrow A_{\mathbb{L}} \rightarrow B_{\mathbb{L}} \rightarrow C_{\mathbb{L}} \rightarrow 0
$$

Proof. 1. Put $A^{\prime}:=\operatorname{ker}(B \rightarrow C)$. The exact sequence

$$
0 \rightarrow A^{\prime} \rightarrow B \rightarrow C \rightarrow 0
$$

then induces for every integer $n \in \mathbb{N}(\mathbb{L})$ the exact sequence

$$
{ }_{n} C \rightarrow A^{\prime} / n \rightarrow B / n \rightarrow C / n \rightarrow 0 .
$$

Because the transition maps of the system $\left(A^{\prime} / n\right)_{n \in \mathbb{N}(\mathbb{L})}$ are all surjective, this is also true for the system $\left(Q_{n}\right)_{n \in \mathbb{N}(\mathbb{L})}$, where $Q_{n}:=\operatorname{ker}(B / n \rightarrow C / n)$. So $\left(Q_{n}\right)_{n \in \mathbb{N}(\mathbb{L})}$ satisfies the Mittag-Leffler condition and we get an exact sequence

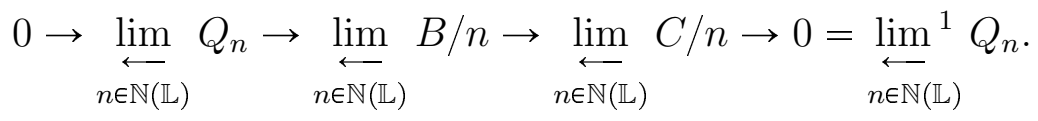

So $B_{\mathbb{L}} \rightarrow C_{\mathbb{L}}$ is surjective. 
2. If, furthermore, we put $D_{n}:=\operatorname{ker}\left(A^{\prime} / n \rightarrow B / n\right)$, the finiteness of all $A / n$ or the Mittag-Leffler condition on $\left({ }_{n} C\right)_{n \in \mathbb{N}(\mathbb{L})}$ induce the Mittag-Leffler condition on the system $\left(D_{n}\right)_{n \in \mathbb{N}(\mathbb{L})}$. So we get an exact sequence

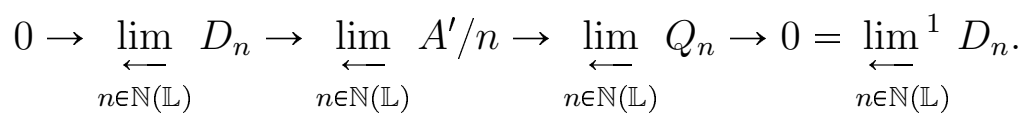

With the surjection $A_{\mathbb{L}} \rightarrow A_{\mathbb{L}}^{\prime}$ and the sequence from the first point, we get the exact sequence

$$
A_{\mathbb{L}} \rightarrow B_{\mathbb{L}} \rightarrow C_{\mathbb{L}} \rightarrow 0
$$

3. Here we have $A \cong A^{\prime}$ and the trivial Mittag-Leffler condition on $\left({ }_{n} C\right)_{n \in \mathbb{N}(\mathbb{L})}$ induces the trivial Mittag-Leffler condition on $\left(D_{n}\right)_{n \in \mathbb{N}(\mathbb{L})}$, such that $\lim _{\leftarrow \in \mathbb{N}(\mathbb{L})} D_{n}$ and $\lim _{\leftarrow}^{1} D_{n \in \mathbb{N}(\mathbb{L})} D_{n}$ vanish in the sequence above. So we have the isomorphism

$$
\lim _{n \in \mathbb{N}(\mathbb{L})} A / n \cong \lim _{n \in \mathbb{N}(\mathbb{L})} Q_{n}
$$

and we get the exact sequence

$$
0 \rightarrow A_{\mathbb{L}} \rightarrow B_{\mathbb{L}} \rightarrow C_{\mathbb{L}} \rightarrow 0 .
$$

Lemma 3.2.11. Let $A$ be an abelian torsion group.

- Then $A$ is the direct sum of its $\ell$-torsion subgroup, i.e. the natural map

$$
\bigoplus_{\ell} A_{\ell \text {-tors }} \stackrel{\sim}{\rightarrow} A
$$

where the sum is taken over all primes $\ell$, is an isomorphism.

- Furthermore, for every set of primes $\mathbb{L}$ we have the isomorphism

$$
A_{\mathbb{L}} \cong \prod_{\ell \in \mathbb{L}} A_{\{\ell\}},
$$

where

$$
A_{\{\ell\}}=\lim _{m \in \mathbb{N}} A / \ell^{m}=A_{\ell \text {-tors },\{\ell\}}=A_{\ell \text {-tors }, \mathbb{L}}
$$

- If $A$ is torsion of finite exponent $n$, then $A \cong A_{\mathbb{L}}$ for every set of primes $\mathbb{L}$ with $n \in \mathbb{N}(\mathbb{L})$. 
Proof. The first statement is clear. The second one uses that $n$ is invertible on $A_{\ell \text {-tors }}$ if $\ell$ does not divide $n$. So $A / n \cong \prod_{\ell \mid n} A_{\ell \text {-tors }} / n$ and the projective limit gives the claim. For the last point note that $A=A / n=A_{\mathbb{L}}$.

Lemma 3.2.12 (Direct sums of torsion and divisible groups).

1. The following properties of an abelian group $A$ are equivalent:

- The quotient of $A$ by its maximal divisible subgroup is a torsion group.

- A is the direct sum of its maximal divisible subgroup and a torsion group.

- $A$ is the direct sum of a divisible group and a torsion group.

2. If $B$ is the direct sum of a torsion and a divisible group, then every quotient of $B$ is.

3. If $C$ is a torsion group and $A$ is the direct sum of a torsion and a divisible group and

$$
0 \rightarrow A \rightarrow B \rightarrow C \rightarrow 0
$$

is exact, then $B$ is also the direct sum of a torsion and a divisible group.

4. If $C$ is a torsion group with $C_{\text {Div }}=0$, e.g. if $C$ is of finite exponent, and

$$
0 \rightarrow A \rightarrow B \rightarrow C \rightarrow 0
$$

is exact, then $A$ is the direct sum of a torsion and a divisible group if and only if $B$ is.

Proof. Easy calculation. See CT93 Lem./Def. 3.1. For example consider the last point: $A_{\text {Div }}$ injects into $B_{\text {Div }}$ and $B_{\text {Div }}$ maps to $C_{\text {Div }}=0$. So $A_{\text {Div }}=B_{\text {Div }}$ and we get an exact sequence

$$
0 \rightarrow A / A_{\text {Div }} \rightarrow B / B_{\text {Div }} \rightarrow C \rightarrow 0,
$$

from which follows that $A / A_{\text {Div }}$ is torsion if and only if $B / B_{\text {Div }}$ is torsion, since $C$ is torsion.

Lemma 3.2.13. Let $\mathbb{L}$ be a set of prime numbers and let

$$
0 \rightarrow D \rightarrow A \stackrel{j}{\rightarrow} T \rightarrow 0
$$

be an exact sequence of abelian groups, where $D$ is $\mathbb{L}$-divisible and $T$ is torsion. Write $T=T_{\mathbb{L} \text {-tors }} \oplus T^{\prime}$ where $T_{\mathbb{L} \text {-tors }}$ is $\mathbb{L}$-torsion and $T^{\prime}$ is $\mathbb{L}$-torsion free. Put $D^{\prime}=j^{-1}\left(T^{\prime}\right)$. Then $D^{\prime} \supseteq D$ is $\mathbb{L}$-divisible and we have

$$
A \cong D^{\prime} \oplus T_{\mathbb{L} \text {-tors }}
$$


Proof. See [JS03] Lemma 7.8: In the exact sequence

$$
0 \rightarrow D \rightarrow D^{\prime} \stackrel{j}{\rightarrow} T^{\prime} \rightarrow 0
$$

both $D$ and $T^{\prime}$ are $\mathbb{L}$-divisible, so $D^{\prime}$ is, too. Therefore the exact sequence

$$
0 \rightarrow D^{\prime} \rightarrow A \stackrel{j}{\rightarrow} T_{\mathbb{L} \text {-tors }} \rightarrow 0
$$

splits, since every $\ell$-primary torsion part of this sequence is exact and splits, using the fact that $\ell$-divisible groups are injective $\mathbb{Z}_{\ell}$-modules for all $\ell \in \mathbb{L}$ and $D^{\prime}$ is $\mathbb{L}$-divisible.

Lemma 3.2.14. Let $\mathbb{L}$ be a set of prime numbers and $A$ be an abelian group whose $\mathbb{L}$-completion $A_{\mathbb{L}}$ has torsion $A_{\mathbb{L}, \mathbb{L} \text {-tors }}$ of finite exponent. Furthermore, assume one of the following assumptions:

- $A$ is the direct sum of a $\mathbb{L}$-divisible group and a torsion group, or

- $A_{\mathbb{L}}$ is torsion of finite exponent.

Then $A_{\mathbb{L}-\text { Div }}=A_{\mathbb{L} \text {-div }}$ and $A_{\mathbb{L}}=A_{\mathbb{L}, \mathbb{L} \text {-tors }}$ is of finite exponent and

$$
A \cong A_{\mathbb{L} \text {-Div }} \oplus A_{\mathbb{L}, \mathbb{L} \text {-tors }}
$$

Proof. Consider the exact sequence

$$
0 \longrightarrow A_{\mathbb{L}-\operatorname{div}} \longrightarrow A \stackrel{f}{\longrightarrow} A_{\mathbb{L}} .
$$

Since $A_{\mathbb{L} \text {-Div }} \subseteq A_{\mathbb{L} \text {-div }}$, in both cases $f$ factors through $A_{\mathbb{L}, \mathbb{L} \text {-tors }}$ which is torsion of finite exponent. By the second point of 3.2 .9 with $B=A_{\mathbb{L}, \mathbb{L} \text {-tors }}$ and the assumption on a finite exponent of $A_{\mathbb{L}, \mathbb{L} \text {-tors }}$ we get $A_{\mathbb{L} \text {-Div }}=A_{\mathbb{L} \text {-div }}$. So $A / A_{\mathbb{L} \text {-Div }}$ then injects into $A_{\mathbb{L}, \mathbb{L} \text {-tors }}$ and is therefore torsion of finite exponent. So we get $\left(A / A_{\mathbb{L} \text {-Div }}\right)_{\mathbb{L}}=A / A_{\mathbb{L} \text {-Div }}$. Since $A_{\mathbb{L}}=\left(A / A_{\mathbb{L} \text {-Div }}\right)_{\mathbb{L}}$ we get $A_{\mathbb{L}}=A / A_{\mathbb{L} \text {-Div }}$ is torsion of finite exponent. So by 3.2 .13 we have $A \cong A_{\mathbb{L} \text {-Div }} \oplus A_{\mathbb{L}}$ (and $f$ is surjective).

Lemma 3.2.15. Let $\mathbb{L}$ be set of prime numbers and let $\mathbb{L}^{\prime}$ be the complementary set of prime numbers. Let $A$ be an abelian group, $A_{\mathbb{L}}=\lim _{n \in \mathbb{N}(\mathbb{L})} A / n$ and $D:=$ $\operatorname{coker}\left(A \stackrel{\iota}{\rightarrow} A_{\mathbb{L}}\right)$. Then we have:

- $A_{\mathbb{L}}$ is uniquely $\mathbb{L}^{\prime}$-divisibel and $D$ is $\mathbb{L}^{\prime}$-divisible.

- Assume that $A / n$ is finite for every integer $n \in \mathbb{N}(\mathbb{L})$. Then we have a canonical isomorphism

$$
A / n \cong A_{\mathbb{L}} / n
$$

for all $n \in \mathbb{N}(\mathbb{L})$ and $D$ is a divisible abelian group, i.e. an injective $\mathbb{Z}$-module. 
- If, furthermore, for every $\ell \in \mathbb{L}$ there exists an integer $m=\ell^{\nu}$, such that the $\ell$-torsion subgroup $A_{\mathbb{L}, \ell \text {-tors }}$ of $A_{\mathbb{L}}$ is killed by $m$, then $D$ is $\mathbb{L}$-torsion free.

Proof. $\quad-A_{\mathbb{L}}$ is a $\mathbb{Z}_{\mathbb{L}}$-module and every integer $m \in \mathbb{N}\left(\mathbb{L}^{\prime}\right)$ has no prime divisor in $\mathbb{L}$ and is therefore invertible in $\mathbb{Z}_{\mathbb{L}}=\prod_{\ell \in \mathbb{L}} \mathbb{Z}_{\ell}$. So for any $a \in A_{\mathbb{L}}$ we have a unique element $b=m^{-1} \cdot a$ with $a=m \cdot b$. So $A_{\mathbb{L}}$ is uniquely $\mathbb{L}^{\prime}$-divisible and as a factor of $A_{\mathbb{L}}$ the group $D$ is also $\mathbb{L}^{\prime}$-divisible.

- For every integer $n \in \mathbb{N}(\mathbb{L})$ the compositum of the natural maps

$$
A \rightarrow A_{\mathbb{L}}=\lim _{m \in \mathbb{N}(\mathbb{L})} A / m \rightarrow A / n
$$

factors through

$$
A / n \rightarrow A_{\mathbb{L}} / n \rightarrow A / n,
$$

which is the identity map. Therefore, $A / n \rightarrow A_{\mathbb{L}} / n$ is injective. Since the image of $A$ in $A_{\mathbb{L}}$ is dense, the image of $A / n \rightarrow A_{\mathbb{L}} / n$ is also dense. But with the finiteness of $A / n$, the image of $A / n$ in $A_{\mathbb{L}} / n$ is also finite. Because $A_{\mathbb{L}}=\lim _{\longleftarrow} A / m$ is pro-finite and hence a Hausdorff group, the finite image of $A / n$ is a closed set in $A_{\mathbb{L}} / n$. So $A / n \rightarrow A_{\mathbb{L}} / n$ is also a surjection. Hence we have isomorphisms of finite groups

$$
A / n \cong \iota(A) / n \cong A_{\mathbb{L}} / n,
$$

where $\iota(A)$ is the image of $A$ in $A_{\mathbb{L}}$. For every integer $n \in \mathbb{N}(\mathbb{L})$ the short exact sequence

$$
0 \longrightarrow \iota(A) \longrightarrow A_{\mathbb{L}} \longrightarrow D \longrightarrow 0
$$

now induces the exaxt sequence

$$
0 \longrightarrow{ }_{n} \iota(A) \longrightarrow{ }_{n} A_{\mathbb{L}} \longrightarrow{ }_{n} D \longrightarrow(A) / n \stackrel{\sim}{\longrightarrow} A_{\mathbb{L}} / n \longrightarrow D / n \longrightarrow 0 .
$$

So $D / n=0$ for every $n \in \mathbb{N}(\mathbb{L})$, and $D$ is $\mathbb{L}$-divisible. With the first point follows that $D$ is also divisible.

- The $\ell$-torsion subgroup $D_{\ell \text {-tors }}=\bigcup_{m \in \mathbb{N}(\{\ell\})} D$ of $D$ is then $\ell$-divisible, too: If $a \in{ }_{m} D$, then there is an $b \in D$ with $n b=a$, so $b \in{ }_{n m} D$.

The surjections ${ }_{n} A_{\mathbb{L}} \rightarrow{ }_{n} D$ from above induce a surjection

$$
A_{\mathbb{L}, \ell \text {-tors }} \rightarrow D_{\ell \text {-tors }}
$$

If we assume to have an integer $m=\ell^{\nu}$ with $m \cdot A_{\mathbb{L}, \ell \text {-tors }}=0$, then $D_{\ell \text {-tors }}$ is also killed by $m$. So we have

$$
D_{\ell \text {-tors }}=D_{\ell \text {-tors }} / m=0
$$


from the $\ell$-divisibility of $D_{\ell \text {-tors }}$. Therefore $D_{\ell \text {-tors }}$ vanishes for every $\ell \in \mathbb{L}$. If there were an element $x$ with $n x=0$ and $n=\ell_{1}^{e_{1}} \cdots \ell_{s}^{e_{s}}$ with $\ell_{i} \in \mathbb{L}$, then $\ell_{2}^{e_{2}} \cdots \ell_{s}^{e_{s}} x \in D_{\ell_{1} \text {-tors }}=0$. So inductively we get $x=0$. Therefore, $D$ is $\mathbb{L}$-torsion free.

\subsection{Good divisors and alterations}

In this section we will collect some fundamental theorems, which we will use later.

Definition 3.3.1. $\quad$ 1. Let $k$ be a field and $Y$ be a simple normal crossing variety over $k$. A good divisor on $Y$ is a reduced closed subscheme $Z \subseteq Y$ of pure codimension 1 such that $Z$ intersects every $k$-fold intersection

$$
Y_{i_{0}, i_{1}, \ldots, i_{k}}:=Y_{i_{0}} \times_{Y} Y_{i_{1}} \times_{Y} \ldots \times_{Y} Y_{i_{k}}
$$

of the irreducible components $Y_{i}$ of $Y$ transversally for any integer $k \geqslant 0$ and such that $U:=Y \backslash Z$ is affine.

2. Let $A$ be discrete valuation ring with residue field $k$ and $\mathfrak{X}$ be a regular, separated and flat scheme of finite type over $S=\operatorname{Spec}(A)$ with strict semi-stable reduction $Y=\mathfrak{X}_{s}$ and of pure relative dimension $d \geqslant 1$. Then a divisor $Z \subseteq \mathfrak{X}$ is called good divisor, if it is flat over $S$ and if the special fibre $Z_{s}$ is a good divisor of $Y$.

Theorem 3.3.2. $\quad$ 1. Let $k$ be a field and $Y$ be simple normal crossing variety over $k$.

- If $Z$ is a good divisor of $Y$, then it is a simple normal crossing variety and the intersections $Y_{i} \cap Z$ are the connected components of $Z$, unless $\operatorname{dim} Y_{i}=1$.

- If $Y$ is proper over $k$ and $Z$ is a good divisor of $Y$ with open complement $U=Y \backslash Z$, then one has

$$
\mathrm{H}_{a-1}^{e ́ t}(U \mid k, \mathbb{Z} / n)=0=\mathrm{H}_{a}^{e ́ t}(\bar{U} \mid k, \mathbb{Z} / n),
$$

for every $a<\operatorname{dim} Y$ and every integer $n \geqslant 1$

- If $Y$ is projective over $k$, then a good divisor always exists.

2. Let $A$ be a henselian discrete valuation ring with residue field $k$ and $\mathfrak{X}$ be a regular, separated and flat scheme of finite type over $S=\operatorname{Spec}(A)$ with strict semi-stable reduction $Y=\mathfrak{X}_{s}$ and of pure relative dimension $d \geqslant 1$.

- If $Z$ is a good divisor of $\mathfrak{X}$, then it is regular and has strict semi-stable reduction, and is of pure relative dimension $d-1$. 
- If $\mathfrak{X}$ is proper over $S, k$ is finite and $Z$ is a good divisor of $\mathfrak{X}$ with open complement $U=\mathfrak{X} \backslash Z$, then one has

$$
\mathrm{H}_{a-2}^{e ́ t}\left(U_{\eta} \mid S, \mathbb{Z} / n(-1)\right)=0=\mathrm{H}_{a-3}^{e ́ t}\left(U_{s} \mid S, \mathbb{Z} / n(-1)\right)
$$

for any integer $n$ and every $a<d=\operatorname{dim} Y$. Furthermore, the residue map

$$
\Delta: \mathrm{H}_{d-2}^{e ́ t}\left(U_{\eta} \mid S, \mathbb{Z} / n(-1)\right) \stackrel{\sim}{\rightarrow} \mathrm{H}_{d-3}^{e ́ t}\left(U_{s} \mid S, \mathbb{Z} / n(-1)\right)
$$

is an isomorphism for every integer $n$ prime to char $(k)$, and is surjective for the case $\operatorname{char}(K)=0$, if one replaces $\mathbb{Z} / n(-1)$ by $\mathbb{Q}_{p} / \mathbb{Z}_{p}(-1)$, where $p=\operatorname{char}(k)$.

- If $\mathfrak{X}$ is projective over $S$ and the generic fibre $X=\mathfrak{X}_{\eta}$ is smooth over the quotient field $K$ of $A$, then a good divisor always exists.

Proof. See JS03 Lem. 3.4, Thm. 3.5, Prp. 4.3, Thm. 4.4. For the existence of the good divisors one needs the Bertini theorems from [JS09] Thm. 1 and Cor. 1, cf. [Poo04], [Jou83] and [Har77]. To avoid finite field extension for finite fields, one has to replace the projective embeddings by multiple ones.

Theorem 3.3.3 (Gabber's refinement of de Jong's alterations). 1. Let $X$ be a scheme separated and of finite type over a field $k, Z \subseteq X$ a nowhere dense closed subset, $\ell \neq \operatorname{char}(k)$ a prime. Then there exists a finite extension $k^{\prime}$ of $k$ of order prime to $\ell$ and an $\ell^{\prime}$-alteration $g: X^{\prime} \rightarrow X$ over $\operatorname{Spec}\left(k^{\prime}\right) \rightarrow \operatorname{Spec}(k)$, (i.e. a proper surjective map sending generic points to generic points such that the corresponding residue field extensions at the generic points are finite with degree prime to $\ell$ ), with $X^{\prime}$ smooth and quasi-projective over $k^{\prime}$ and $g^{-1}(Z)$ the support of a simple normal crossings divisor.

2. Let $S$ be an excellent trait, $X$ a scheme separated and of finite type over $S, \ell$ a prime invertible on $S, Z \subseteq X$ a nowhere dense closed subset. Then there exists a finite extension $S^{\prime} \rightarrow S$ of degree prime to $\ell$ and an $\ell^{\prime}$-alteration $g: X^{\prime} \rightarrow X$ over $S^{\prime} \rightarrow S$, a simple normal crossing divisor $T^{\prime}$ in $X^{\prime}$ such that $X^{\prime}$ is regular and quasi-projective over $S^{\prime}, Z^{\prime}=g^{-1}(Z)$ is a subdivisor of $\left(X_{s^{\prime}}^{\prime}\right)_{\mathrm{red}} \cup T^{\prime},\left(s^{\prime}\right.$ denoting the closed point of $\left.S^{\prime}\right)$, with $\left(X^{\prime}, T^{\prime}\right)$ étale locally given by $X^{\prime}=S^{\prime}\left[t_{1}, \ldots, t_{n}\right] /\left(t_{1}^{a_{1}} \cdots t_{r}^{a_{r}}-\pi\right), \pi$ a uniformizing parameter in $S^{\prime}, \operatorname{gcd}\left(p, a_{1}, \ldots, a_{r}\right)=1, p=\operatorname{char}(k), k$ the residue field of $S$, and $T^{\prime}=$ $S^{\prime}\left[t_{1}, \ldots, t_{n}\right] /\left(t_{r+1} \cdots t_{m}\right)$.

Proof. See [Ill09] Thm. 1.3 and Thm. 1.4, cf. dJ96]. 


\subsection{The structure of $S K_{1}$ and the kernel of the reciprocity map}

In this section we will transfer the results from the previous sections, where we analyzed the reciprocity map modulo $n$, to the usual reciprocity map. Additionally we are able to determine the structure of $\operatorname{SK}_{1}(X)$, or the kernel of the norm map, resp., for varieties $X$ over local fields in any dimension. Since the kernel of the reciprocity map lies in the kernel of the norm map with finite index, we will find, that in all dimension it is a direct sum of a finite group and a $\mathbb{L}$-divisible group, where $\mathbb{L}$ is a set of prime numbers not containing the residue characteristic $p$.

Theorem 3.4.1. Let $K$ be the quotient field of a henselian discrete valuation ring with finite residue field $k$ and let $X$ be a smooth proper geometrically connected variety over $K$. Let $\pi_{1}^{\mathrm{geo}}(X):=\operatorname{ker}\left(\pi_{1}^{\mathrm{ab}}(X) \rightarrow G_{K}^{\mathrm{ab}}\right)$ be the kernel of the norm map. Then we have:

$$
\pi_{1}^{\mathrm{geo}}(X) \cong T \oplus \hat{\mathbb{Z}}^{r}
$$

where $T$ is finite and $r$ is the k-rank of the special fiber of the Néron model of the Albanese variety of $X$.

Proof. See [JS03] Theorem 7.1. The prime-to-char $(k)$-part is due to Grothendieck and the char $(k)$-part comes from Yoshida Yos03.

Lemma 3.4.2. Let $K$ be a henselian valuation field with finite residue field.

1. If $f: V \rightarrow M$ is a proper surjective morphism with $V$ and $M$ of finite type over $K$, then the push-forward map, called norm map, has coker $\left(f_{*}: \operatorname{SK}_{1}(V) \rightarrow\right.$ $\left.\mathrm{SK}_{1}(M)\right)$ of finite exponent.

2. If, furthermore, $M=\operatorname{Spec}(K)$ and $V$ is non-empty, then coker $N_{V \mid K}:=$ coker $f_{*}$ is finite.

Proof. See [JS03] Lem. 6.2: It uses that norm groups are of finite index in $K^{\times}$, and that for a finite and flat morphism, there is a pull-back map $f^{*}$ with $f_{*} \circ f^{*}=$ $[\kappa(V): \kappa(M)]$.

Proposition 3.4.3. Let $K$ be a henselian valuation field with finite residue field.

1. Let $V$ be a geometrically irreducible and proper variety over $K$. Assume one of the following conditions:

- $\operatorname{char}(K)=0$,

- $V$ is smooth and projective over $K$.

Then the kernel $\operatorname{ker}\left(N_{V \mid K}\right)$ of the norm map $N_{V \mid K}: \mathrm{SK}_{1}(V) \rightarrow K^{\times}$is a direct sum of a divisible group and a torsion group. 
2. If $U$ is an open subset of such a $V$ with $U \neq V$ and $\operatorname{dim} U \geqslant 1$, then $\operatorname{SK}_{1}(U)$ is a direct sum of a divisible group and a torsion group.

3. If $V$ is in addition a curve over $K$, i.e. $\operatorname{dim} V=1$, then we can replace "torsion" by "finite" in the statements above.

Proof. Cf. Sat05] Prp. 2.3 and [JS03 Prp. 6.3. The statement is step by step reduced to a curve. If $V$ is a geometrically irreducible, smooth and proper curve (= projective curve by [Liu02] Ex.7.5.4), the problem is solved in [Sai85a] Thm. 4.1 and [Yos03] Thm. 5.1, respectively, for local fields. But the whole proof also works for henselian valuation fields with finite residue fields. Now let $\operatorname{char}(K)=0$ :

- If $V$ is a geometrically irreducible proper, but not smooth, curve over the perfect field $K$, then the normalisation map $V^{\prime} \rightarrow V$ is finite and birational and $V^{\prime}$ is a geometrically irreducible, projective and smooth curve over $K$, since $\operatorname{char}(K)=0$. Let $Z^{\prime}$ be the exceptional divisor, which consists only of a finite set of points, and let $Z$ be its image in $V$. Then the cokernel coker 1 of $\mathrm{SK}_{1}\left(Z^{\prime}\right) \rightarrow \mathrm{SK}_{1}(Z)$ is finite, since it is the cokernel of a finite sum of norm maps between finite field extensions of $K$. With $V^{\prime} \backslash Z^{\prime} \cong V \backslash Z$, we get a commutative diagram of exact sequences

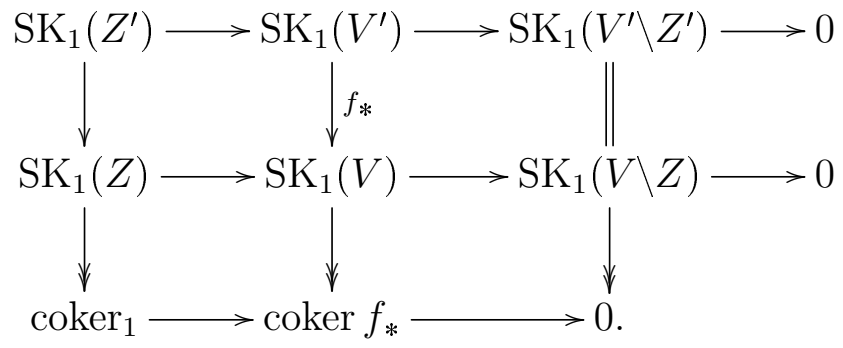

So we have a surjection coker $_{1} \rightarrow \operatorname{coker}_{*}$, showing that coker $f_{*}$ is finite. And we have an exact diagram

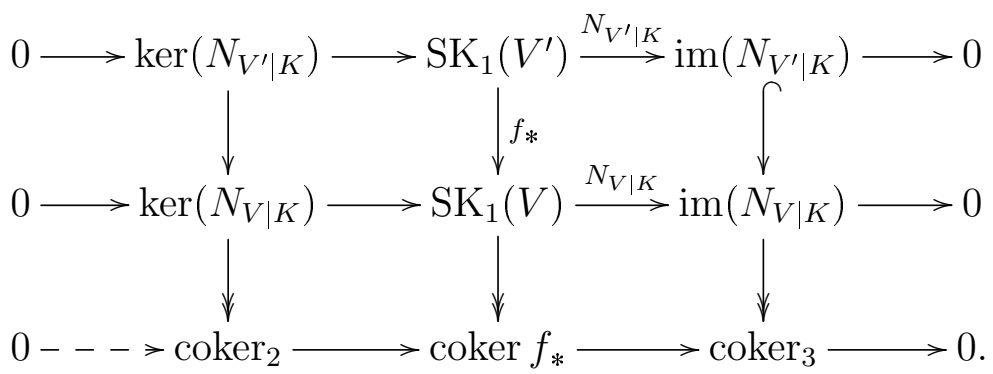

So coker 2 is also finite. Therefore with $\operatorname{ker}\left(N_{V^{\prime} \mid K}\right)$, the group $\operatorname{ker}\left(N_{V \mid K}\right)$ is a direct sum of a finite group and a divisible group, too, by 3.2.12. 
- Now let $V$ be a geometrically irreducible projective variety over $K$ of dimension $\geqslant 2$ and let $\alpha \in \operatorname{ker}\left(N_{V \mid K}\right)$ be represented by a finite sum $\sum_{x \in V_{0}} a_{x}$ with $a_{x} \in \kappa(x)^{\times}$. Following Salberger's argument (c.f. the proof of [CT93] Prp. 3.3) we take a finite normal extension $L$ of $K$, in which every of the finite number of points $x$ with $a_{x} \neq 1$ becomes $L$-rational. So every preimage $y \in V_{L}:=V \otimes_{K} L$ of such a $x$ under the map $f: V_{L} \rightarrow V$ is $L$-rational. So by a Bertini theorem ([Jou83] Cor. I.6.11, Thm. II.5.1, resp.) there is a geometrically irreducible and projective curve $C$ in $V_{L}$ over $L$ running through all the finite number of points $y$ which maps to the points $x$ with $a_{x} \neq 1$. Consider the diagram

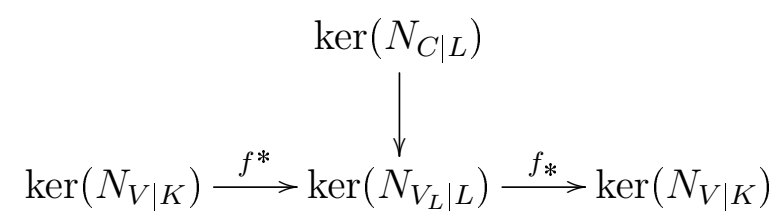

So $f^{*}(\alpha)=\sum_{y \in V_{L, 0}} a_{y}$ lies in the image of $\operatorname{ker}\left(N_{C \mid L}\right)$ with $a_{y}=a_{f(y)} \in \kappa(y)^{\times}$. So by the previous point about curves, there is a natural number $n$ such that $f^{*}(n \alpha)$ lies in the maximal divisible subgroup of $\operatorname{ker}\left(N_{V_{L} \mid L}\right)$. Since the compositum $f_{*} f^{*}$ is multiplication with $m=[L: K]$, we have that

$$
m n \alpha=f_{*} f^{*}(n \alpha)
$$

lies in the maximal divisible subgroup of $\operatorname{ker}\left(N_{V \mid K}\right)$. So modulo the maximal divisible subgroup, every element in $\operatorname{ker}\left(N_{V \mid K}\right)$ is torsion.

- Let $V$ be a geometrically irreducible and proper variety over $K$. Then by Chow's Lemma there is a projective variety $V^{\prime}$ over $K$ with a projective and birational morphism $f: V^{\prime} \rightarrow V$. So $V^{\prime}$ is also geometrically irreducible and we get a commutative diagram of exact sequences

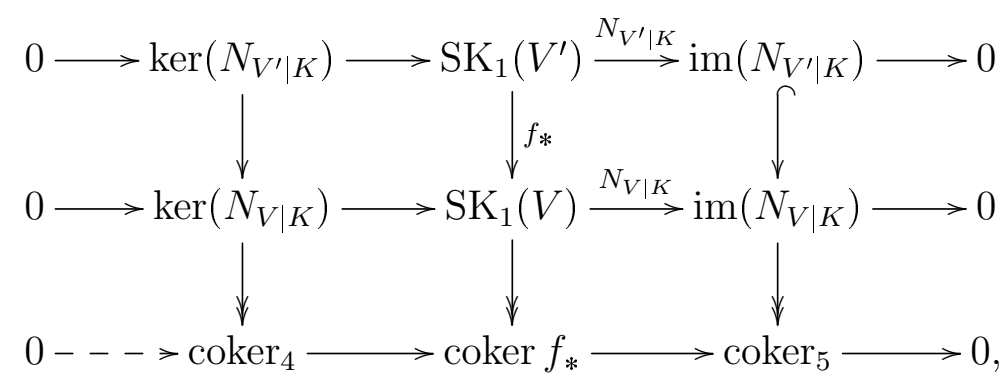

where coker $f_{*}$ is torsion of finite exponent by 3.4.2. Since coker ${ }_{4}$ is also torsion of finite exponent and $\operatorname{ker}\left(N_{V^{\prime} \mid K}\right)$ is the direct sum of a divisible group and a torsion group, this also holds for $\operatorname{ker}\left(N_{V \mid K}\right)$ by 3.2 .12 . 
- If $U$ is a proper open subset of such a $V$ with closed complement $Z$ then we have the diagram:

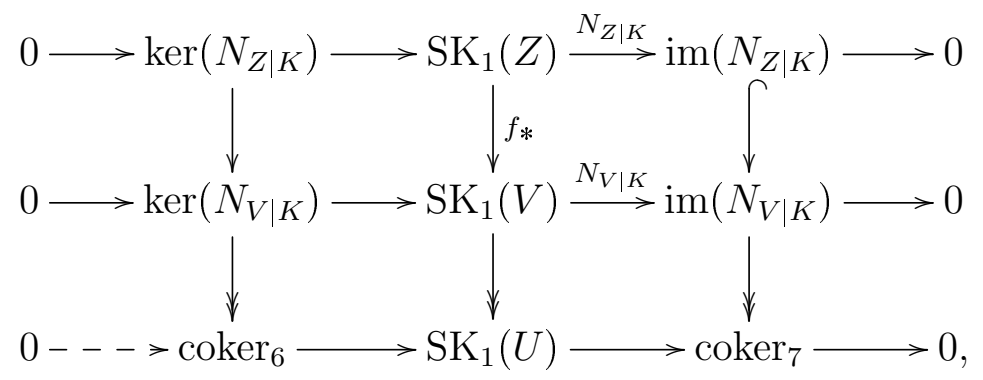

where coker $_{7}$ is finite as a quotient of norm groups in $K^{\times}$. As the image of $\operatorname{ker}\left(N_{V \mid K}\right)$ the group coker $_{6}$ is the direct sum of a divisible and torsion group. And the image of coker 6 has finite index in $\operatorname{SK}_{1}(U)$. Therefore $\operatorname{SK}_{1}(U)$ is also the direct sum of a divisible and a torsion group by 3.2 .12 .

For the other case we use the same methods. Note that the normalisation of a curve is not smooth in general (cf. Har77 Ex. III.10.1) if we drop the assumption $\operatorname{char}(K)=0$. Therefore, we have to assume smoothness. Since Chow's Lemma could lead to singularities, we also have to assume projectivity. Luckily, Bertini's Theorem preserves smoothness, so that all the arguments work in this restricted case.

Remark 3.4.4. If one tries to proof that the $\mathbb{L}$-completion of the kernel of the norm map is torsion by the same methods, then one is faced with the problem that an element of $\mathrm{SK}_{1}(X)_{\mathbb{L}}$ is not given by a finite sum over closed points of some elements from $\kappa(x)_{\mathbb{L}}^{\times}$, because the $\mathbb{L}$-completion does not commute with direct sums. So Salberger's argument does not work in that case.

Lemma 3.4.5. Let $K$ be the quotient field of a henselian discrete valuation ring with finite residue field $k$ and let $X$ be a smooth proper geometrically connected variety over $K$. Assume that $\operatorname{char}(K)=0$ or that $X$ is projective over $K$. Let $V(X):=\operatorname{ker}\left(\mathrm{SK}_{1}(X) \stackrel{N}{\rightarrow} K^{\times}\right)$be the kernel of the norm map. Then we have:

1. $\rho^{X}(V(X))$ is a finite subgroup of $\pi_{1}^{\text {geo }}(X)$.

2. $\operatorname{ker} \rho^{X}$ and $V(X)$ are the direct sums of the divisible group $D=\operatorname{SK}_{1}(X)_{\text {Div }}$ and torsion groups.

3. $\left(\operatorname{ker} \rho^{X}\right)_{\mathbb{L}}$ equals $\operatorname{ker}\left(\rho_{\mathbb{L}}^{X}\right)$ for every set of prime numbers $\mathbb{L}$, i.e. we have

$$
\lim _{n \in \mathbb{N}(\mathbb{L})}\left(\operatorname{ker} \rho^{X}\right) / n=\lim _{n \in \mathbb{N}(\mathbb{L})} \operatorname{ker} \rho_{n}^{X} .
$$


4. $\operatorname{coker}\left(\rho^{X}\right)$ has a finite torsion subgroup.

Proof. 1. See [JS03 Theorem 7.2 and cf. Szza00. The first statement follows from 3.4.3, which shows that $V(X)$ is the direct sum of a torsion group and a divisible group. So the image of $V(X)$ is torsion and by the structure of $\pi_{1}^{\mathrm{geo}}(X)$ in 3.4 .1 finite.

2. Consider the reciprocity map $\rho^{X}: \mathrm{SK}_{1}(X) \rightarrow \pi_{1}^{\mathrm{ab}}(X)$. We have

$$
\rho^{X}\left(\mathrm{SK}_{1}(X)_{\text {Div }}\right) \subseteq \pi_{1}^{\mathrm{ab}}(X)_{\text {Div }}=0
$$

since $\pi_{1}^{\mathrm{ab}}(X)$ is a pro-finite group. So $\operatorname{SK}_{1}(X)_{\text {Div }} \subseteq \operatorname{ker} \rho^{X}$ is the maximal divisible subgroup of $\operatorname{ker} \rho^{X}$. Consider the commutative and exact diagram

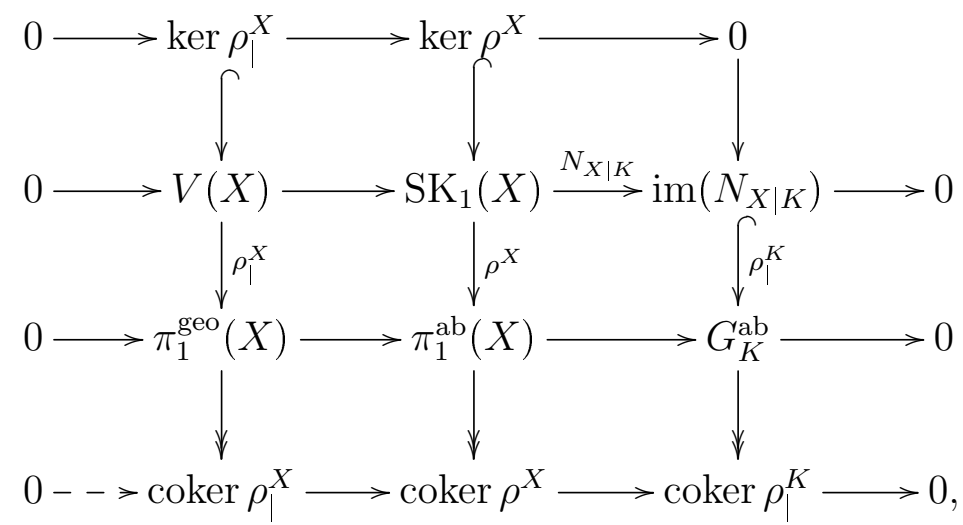

where $\rho_{\mid}^{K}$ denotes the restriction of the local reciprocity map

$$
\rho^{K}: K^{\times} \hookrightarrow G_{K}^{\mathrm{ab}},
$$

which is injective, and beside this has $\operatorname{coker}\left(\rho^{K}\right) \cong \hat{\mathbb{Z}} / \mathbb{Z}$ by local class field theory. It induces the exactness after the dotted line in the diagram. We get a sequence of inclusions of abelian groups

$$
\operatorname{SK}_{1}(X)_{\text {Div }} \subseteq \operatorname{ker} \rho^{X} \subseteq V(X) \subseteq \operatorname{SK}_{1}(X)
$$

It follows that $\left(\operatorname{ker} \rho^{X}\right)_{\text {Div }}=V(X)_{\text {Div }}=\operatorname{SK}_{1}(X)_{\text {Div }}$ and we get an injection

$$
\operatorname{ker} \rho^{X} / \operatorname{SK}_{1}(X)_{\text {Div }} \subseteq V(X) / V(X)_{\text {Div }},
$$

where the last group is torsion by 3.4 .3 .

3. The groups $\pi_{1}^{\mathrm{ab}}(X), \pi_{1}^{\text {geo }}(X), K^{\times}, \operatorname{im}\left(N_{X \mid K}\right)$ have finite torsion subgroups. To show, that coker $\rho^{X}$ only has a finite torsion subgroup, we note that $\rho^{X}(V(X))$ 
is a finite subgroup of $\pi_{1}^{\text {geo }}(X) \cong T \oplus \hat{\mathbb{Z}}^{r}$. So $\operatorname{coker}\left(\rho_{\mid}^{X}\right) \cong T^{\prime} \oplus \hat{\mathbb{Z}}^{r}$ with a finite $T^{\prime}$, has only a finite torsion subgroup. And the local reciprocity map $\rho^{K}$ and the inclusion $\operatorname{im}\left(N_{X \mid K}\right) \subseteq K^{\times}$give an exact diagram

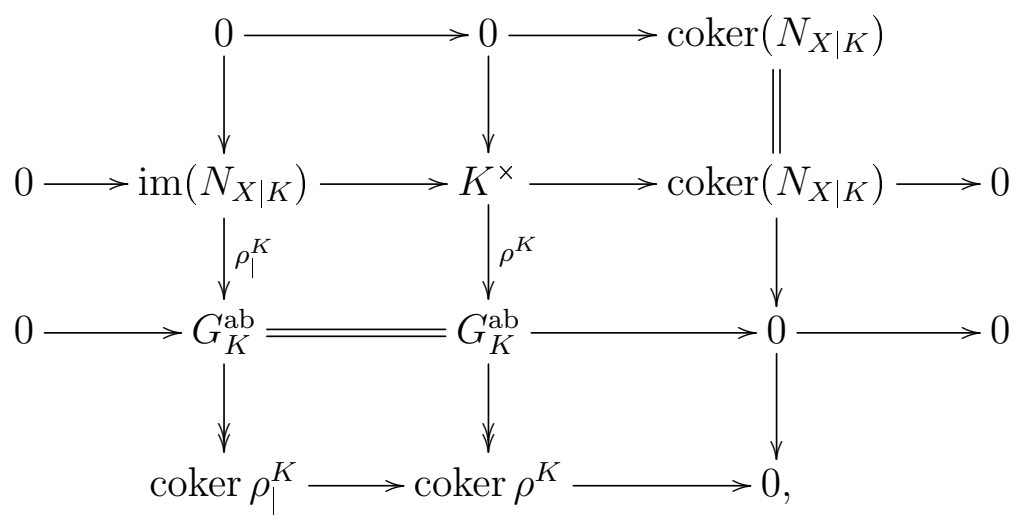

which induce the exact sequence

$$
0 \longrightarrow \operatorname{coker}\left(N_{X \mid K}\right) \longrightarrow \operatorname{coker} \rho_{\mid}^{K} \longrightarrow \operatorname{coker} \rho^{K} \longrightarrow 0
$$

by the snake lemma. Furthermore, coker $\rho^{K} \cong \hat{\mathbb{Z}} / \mathbb{Z}$ is torsion free and $\operatorname{coker}\left(N_{X \mid K}\right)$ is finite by 3.4.2. Therefore, with coker $\rho_{\mid}^{K}$ and coker $\rho_{\mid}^{X}$, the group coker $\rho^{X}$ has only a finite torsion subgroup $\left(\operatorname{coker} \rho^{X}\right)_{\text {tors }}$. So by 3.2.10 the whole first diagram stays exact after any $\mathbb{L}$-completion. In particular we have the equality $\left(\operatorname{ker} \rho^{X}\right)_{\mathbb{L}}=\operatorname{ker}\left(\rho_{\mathbb{L}}^{X}\right)$.

Theorem 3.4.6. Let $X$ be an irreducible, proper and smooth surface, i.e. $\operatorname{dim} X=$ 2 over a local field $K$ and let $\mathbb{L}$ be a set of prime numbers not containing char $(K)$. Then the kernel of the reciprocity map $\operatorname{ker} \rho^{X}$ is the direct sum a finite group and $a \mathbb{L}$-divisible group.

Proof. See [JS03] Thm. 1.8, cf. [Sai85a], Sza00].

Theorem 3.4.7. Let $S=\operatorname{Spec}(A)$ be the spectrum of a henselian discrete valuation ring $A$ with quotient field $K$ and finite residue field $k$. Let $f: \mathfrak{X} \rightarrow S$ be a regular, proper, flat scheme over $S$ with smooth and connected generic fiber $X$ and strict semi-stable reduction $Y$ as the special fiber. Assume moreover that $\operatorname{char}(K)=0$ or that $f$ is projective. Let $Y=\bigcup_{v \in I} Y_{v}$ be a fixed ordering of the irreducible components of $Y$ and let $\Gamma_{Y}$ be the dual complex to $\left(Y,\left(Y_{i}\right)_{i \in I},(I,<)\right)$.

Furthermore, let $\mathbb{L}$ either be a set of prime numbers not containing $p=\operatorname{char}(k)$, or let $\operatorname{char}(K)=0$ and $p \in \mathbb{L}$ with the additionally assumption that the specialisation map

$$
\mathrm{H}_{2}^{K}\left(X \mid S, \mathbb{Z} / p^{\nu}(-1)\right) \cong \mathrm{H}_{2}^{K}\left(Y \mid S, \mathbb{Z} / p^{\nu}(-1)\right)
$$


is a natural isomorphism for all $\nu \geqslant 1$, which by 2.5.9 and 2.5.11 is the case if $f$ is projective and

$$
\mathrm{H}_{4}^{K}\left(\mathfrak{X} \mid S, \mathbb{Q}_{p} / \mathbb{Z}_{p}(-1)\right) \quad \text { is } p \text {-divisible. }
$$

Then we have:

1. For every integer $n \in \mathbb{N}(\mathbb{L})$ the reciprocity map modulo $n$ fits into a commutative diagram of exact sequences of finite groups

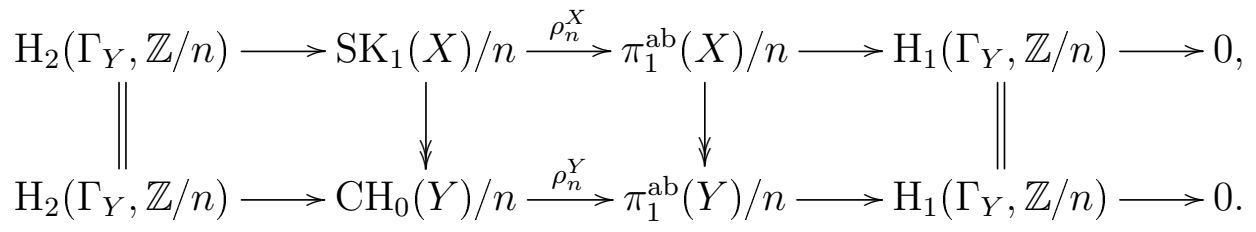

2. The $\mathbb{L}$-completion of the reciprocity map fits into a commutative diagram of exact sequences of finitely generated $\mathbb{Z}_{\mathbb{L} \text {-modules }}$

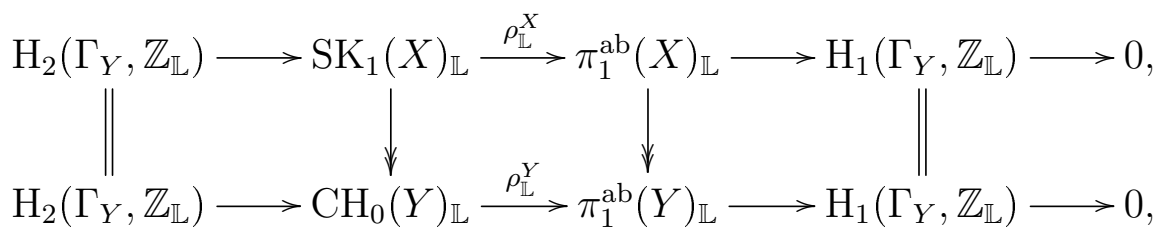

where $\operatorname{ker} \rho_{\mathbb{L}}^{Y}$ and $\operatorname{ker} \rho_{\mathbb{L}}^{X}$ are quotients of $\mathrm{H}_{2}\left(\Gamma_{Y}, \mathbb{Z}_{\mathbb{L}}\right)$. $\operatorname{ker} \rho_{\mathbb{L}}^{Y}$ is finite and equals $\mathrm{H}_{0}\left(\pi_{1}^{\mathrm{ab}}\left(Y^{[\bullet]}\right)_{\mathbb{L}}\right)$.

3. If $f$ is projective, then $\operatorname{ker} \rho^{X}$ and $V(X)$ are the direct sums of their maximal $\mathbb{L}$-divisible subgroup and their $\mathbb{L}$-completion $\operatorname{ker} \rho_{\mathbb{L}}^{X}, V(X)_{\mathbb{L}}$ resp., which is a finite (!) $\mathbb{L}$-torsion group.

4. If, furthermore, $\operatorname{char}(K)=0$ and the above assumption on $p$ holds, then we get

$$
\operatorname{coker} \rho^{X} \cong \mathrm{H}_{1}\left(\Gamma_{Y}, \hat{\mathbb{Z}}\right) \oplus D_{X},
$$

where $D_{X}:=\operatorname{coker}\left(\mathrm{SK}_{1}(X) \stackrel{\iota_{1}}{\rightarrow} \hat{\mathrm{SK}}_{1}(X)\right)$ is a uniquely divisible group, and we get an exact sequence:

$$
\mathrm{H}_{2}\left(\Gamma_{Y}, \hat{\mathbb{Z}}\right) \oplus D^{X} \longrightarrow \mathrm{SK}_{1}(X) \stackrel{\rho^{X}}{\longrightarrow} \pi_{1}^{\mathrm{ab}}(X) \longrightarrow \mathrm{H}_{1}\left(\Gamma_{Y}, \hat{\mathbb{Z}}\right) \oplus D_{X} \longrightarrow 0
$$

where $D^{X}:=\operatorname{SK}_{1}(X)_{\text {Div }}$ is divisible.

Proof. 1. This is a recapitulation of 3.1 .4 . 
2. This conclusion comes from the first point by taking the inverse limit over $\mathbb{N}(\mathbb{L})$. For this, note, that the universal coefficient theorem yields the exact sequences

$$
0 \rightarrow \mathrm{H}_{i}\left(\Gamma_{Y}, \mathbb{Z}\right) \otimes_{\mathbb{Z}} A \rightarrow \mathrm{H}_{i}\left(\Gamma_{Y}, A\right) \rightarrow \operatorname{Tor}_{1}^{\mathbb{Z}}\left(\mathrm{H}_{i-1}\left(\Gamma_{Y}, \mathbb{Z}\right), A\right) \rightarrow 0
$$

for $i=1,2$ and every abelian group $A$. Taking the limit over the projective system given by the groups $A=\mathbb{Z} / n$ for $n \in \mathbb{N}(\mathbb{L})$ the Tor $_{1}$-term vanishes in the limit, since $\mathrm{H}_{i-1}\left(\Gamma_{Y}, \mathbb{Z}\right)$ has a finite torsion subgroup, and we get

$$
\lim _{n \in \mathbb{N}(\mathbb{L})} \mathrm{H}_{i}\left(\Gamma_{Y}, \mathbb{Z} / n\right) \cong \lim _{n \in \mathbb{N}(\mathbb{L})} \mathrm{H}_{i}\left(\Gamma_{Y}, \mathbb{Z}\right) \otimes_{\mathbb{Z}} \mathbb{Z} / n \cong \mathrm{H}_{i}\left(\Gamma_{Y}, \mathbb{Z}\right) \otimes_{\mathbb{Z}} \mathbb{Z} \mathbb{\mathbb { L }}
$$

The last isomorphism is true, because $\mathrm{H}_{i}\left(\Gamma_{Y}, \mathbb{Z}\right)$ is a finitely generated $\mathbb{Z}$ module. So $H_{i}\left(\Gamma_{Y}, \mathbb{Z}\right) \otimes_{\mathbb{Z}} \mathbb{Z}_{\mathbb{L}}$ is a finitely generated $\mathbb{Z}_{\mathbb{L}}$-module. Furthermore, taking $A=\mathbb{Z}_{\mathbb{L}}$ in the coefficients sequence above, we get

$$
\mathrm{H}_{i}\left(\Gamma_{Y}, \mathbb{Z}\right) \otimes_{\mathbb{Z}} \mathbb{Z}_{\mathbb{L}} \cong \mathrm{H}_{i}\left(\Gamma_{Y}, \mathbb{Z}_{\mathbb{L}}\right)
$$

because $\mathbb{Z}_{\mathbb{L}}$ is torsion free, i.e. a flat $\mathbb{Z}$-module. So the stated diagram follows. Since $\pi_{1}^{\mathrm{ab}}(X)$ is a finitely generated pro-finite group and $\mathrm{H}_{2}\left(\Gamma_{Y}, \mathbb{Z}_{\mathbb{L}}\right)$ is a finitely generated $\mathbb{Z}_{\mathbb{L}}$-module, the group $\operatorname{SK}_{1}(X)_{\mathbb{L}}$ also is a finitely generated $\mathbb{Z}_{\mathbb{L}}$-module.

3. Since by 3.4.5 $\rho^{X}(V(X))$ is finite, we have that $\operatorname{ker} \rho^{X} \subseteq V(X)$ is of finite index. So the two statements are equivalent by 3.2.12. We argue by induction on $d=\operatorname{dim} X$. For $d=1$ this is due to [Sai85a] Thm. 4.1 and Yos03] Thm. 5.1 as formulated in 3.4.3. The case $d=2$ is due to JS03 Thm. 1.8, see 3.4.6. And we will follow an analogue strategy for $d \geqslant 3$. Let $Z \subseteq \mathfrak{X}$ be a good divisor from 3.3 .2 and $U=\mathfrak{X} \backslash Z$ be the open complement.

- First of all we show that for almost all $\ell \in \mathbb{L}$ and every $\nu \geqslant 1$ we have that

$$
\operatorname{ker} \rho_{\ell^{\nu}}^{X} \subseteq \operatorname{im}\left(\operatorname{SK}_{1}\left(Z_{\eta}\right) / \ell^{\nu}\right)
$$

Therefore consider the commutative diagram with exact rows coming from the niveau spectral sequence 2.1.6 for the étale homology theory over $S$, where we omit the twist $(-1)$ in the coefficient for brevity, cf. 
3.1 .4

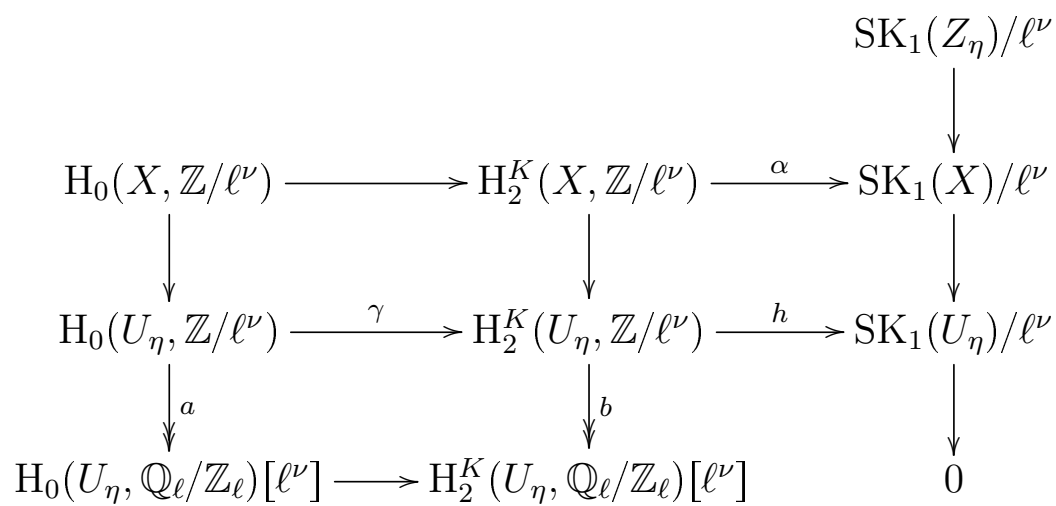

where we can identify $\operatorname{im}(\alpha)$ with $\operatorname{ker} \rho_{\ell^{\nu}}^{X}$ by 3.1.4. The maps $a$ and $b$ are surjective as a part of a coefficient sequence, cf. 2.4.14:

$\mathrm{H}_{3}^{K}\left(U_{\eta}, \mathbb{Q}_{\ell} / \mathbb{Z}_{\ell}(-1)\right) / \ell^{\nu} \hookrightarrow \mathrm{H}_{2}^{K}\left(U_{\eta}, \mathbb{Z} / \ell^{\nu}(-1)\right) \rightarrow \mathrm{H}_{2}^{K}\left(U_{\eta}, \mathbb{Q}_{\ell} / \mathbb{Z}_{\ell}(-1)\right)\left[\ell^{\nu}\right]$.

We now want to show that $\gamma$ is surjective, i.e. $h$ is the zero map, at least for almost all primes $\ell \in \mathbb{L}$.

For $d=2$ this was done in [JS03]: $b$ is an isomorphism by the coefficient sequence above and the vanishing of $\mathrm{H}_{3}^{K}(U \eta)$ by an dimension argument. The bottom map is an isomorphisms by JS03 Lem. 6.5 and 3.3.2 for $\ell \neq p$. So $\gamma$ is then clearly surjective for every $\ell \in \mathbb{L} \backslash\{p\}$.

Now let $d \geqslant 3$ and consider the exact sequence

$$
\mathrm{H}_{0}\left(U_{\eta}, \mathbb{Q}_{\ell} / \mathbb{Z}_{\ell}\right) \longrightarrow \mathrm{H}_{2}^{K}\left(U_{\eta}, \mathbb{Q}_{\ell} / \mathbb{Z}_{\ell}\right) \longrightarrow \mathrm{SK}_{1}\left(U_{\eta}\right) \otimes \mathbb{Q}_{\ell} / \mathbb{Z}_{\ell} .
$$

Since $U_{\eta}$ is the complement of a good divisor and with $d \geqslant 3$, by 3.3 .2 we get

$$
\mathrm{H}_{0}\left(U_{\eta} \mid S, \mathbb{Q}_{\ell} / \mathbb{Z}_{\ell}(-1)\right)=0 .
$$

Furthermore, by 3.4 .3 the group $\operatorname{SK}_{1}\left(U_{\eta}\right)$ is the direct sum of a divisible group and a torsion group, therefore $\mathrm{SK}_{1}\left(U_{\eta}\right) \otimes \mathbb{Q}_{\ell} / \mathbb{Z}_{\ell}$ vanishes, too, from which follows that

$$
\mathrm{H}_{2}^{K}\left(U_{\eta} \mid S, \mathbb{Q}_{\ell} / \mathbb{Z}_{\ell}(-1)\right)=0 .
$$

We show that $b$ is injective for almost all $\ell \in \mathbb{L}$ with help of the coefficient sequence from above. Consider the commutative and exact diagram with $\mathbb{Q}_{\ell} / \mathbb{Z}_{\ell}(-1)$-coefficients:

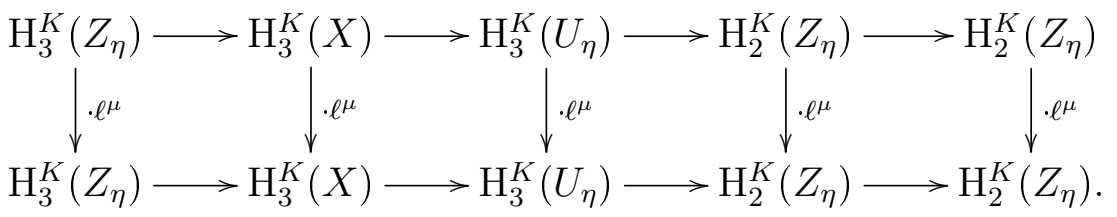


Now by 2.5.8, 2.5.11 resp., and 2.4.13 we have identifications

$$
\begin{aligned}
& \mathrm{H}_{i}^{K}\left(Z_{\eta} \mid S, \mathbb{Q}_{\ell} / \mathbb{Z}_{\ell}(-1)\right) \cong \mathrm{H}_{i}^{K}\left(Z_{s} \mid S, \mathbb{Q}_{\ell} / \mathbb{Z}_{\ell}(-1)\right) \cong \mathrm{H}_{i}\left(\Gamma_{Z_{s}}, \mathbb{Q}_{\ell} / \mathbb{Z}_{\ell}\right), \\
& \mathrm{H}_{i}^{K}\left(X \mid S, \mathbb{Q}_{\ell} / \mathbb{Z}_{\ell}(-1)\right) \cong \mathrm{H}_{i}^{K}\left(Y \mid S, \mathbb{Q}_{\ell} / \mathbb{Z}_{\ell}(-1)\right) \cong \mathrm{H}_{i}\left(\Gamma_{Y}, \mathbb{Q}_{\ell} / \mathbb{Z}_{\ell}\right),
\end{aligned}
$$

for $\ell \neq p=\operatorname{char}(k)$ and the universal coefficient theorem for $\Gamma=\Gamma_{Z_{s}}, \Gamma_{Y}$ :

$$
0 \rightarrow \mathrm{H}_{i}(\Gamma, \mathbb{Z}) \otimes \mathbb{Q}_{\ell} / \mathbb{Z}_{\ell} \rightarrow \mathrm{H}_{i}\left(\Gamma, \mathbb{Q}_{\ell} / \mathbb{Z}_{\ell}\right) \rightarrow \mathrm{H}_{i-1}(\Gamma, \mathbb{Z})_{\ell \text {-tors }} \rightarrow 0 .
$$

So if $\ell$ does not divide the finite order of $\mathrm{H}_{i-1}(\Gamma, \mathbb{Z})_{\text {tors }}$, then the first map is an isomorphism and $\mathrm{H}_{i}\left(\Gamma, \mathbb{Q}_{\ell} / \mathbb{Z}_{\ell}\right)$ is uniquely divisible. Therefore, if $\ell$ does not divide the finite number

$$
p \cdot \# \mathrm{H}_{1}\left(\Gamma_{Z_{s}}, \mathbb{Z}\right)_{\text {tors }} \cdot \# \mathrm{H}_{2}\left(\Gamma_{Z_{s}}, \mathbb{Z}\right)_{\text {tors }} \cdot \# \mathrm{H}_{1}\left(\Gamma_{Y}, \mathbb{Z}\right)_{\text {tors }} \cdot \# \mathrm{H}_{2}\left(\Gamma_{Y}, \mathbb{Z}\right)_{\text {tors }},
$$

the four outer multiplications in the $\ell^{\mu}$-diagram are isomorphisms and the five-lemma yields that $\mathrm{H}_{3}^{K}\left(U_{\eta}, \mathbb{Q}_{\ell} / \mathbb{Z}_{\ell}(-1)\right)$ is uniquely $\ell$-divisible. So

$$
\mathrm{H}_{3}^{K}\left(U_{\eta}, \mathbb{Q}_{\ell} / \mathbb{Z}_{\ell}(-1)\right) / \ell^{\nu}=0
$$

and the map

$$
H_{2}^{K}\left(U_{\eta} \mid S, \mathbb{Z} / \ell^{\nu}(-1)\right) \rightarrow H_{2}^{K}\left(U_{\eta} \mid S, \mathbb{Q}_{\ell} / Z_{\ell}(-1)\right)\left[\ell^{\nu}\right]=0
$$

is injective and therefore both groups are zero. It follows that the map $\gamma$ in the first diagram is surjective and $h$ is the zero map. So, since

$$
\operatorname{SK}_{1}\left(Z_{\eta}\right) / \ell^{\nu} \rightarrow \operatorname{SK}_{1}(X) / \ell^{\nu} \rightarrow \operatorname{SK}_{1}\left(U_{\eta}\right) / \ell^{\nu} \rightarrow 0
$$

is exact and $h=0$, we get that

$$
\operatorname{ker} \rho_{\ell^{\nu}}^{X}=\operatorname{im} \alpha \subseteq \operatorname{ker}\left(\operatorname{SK}_{1}(X) / \ell^{\nu} \rightarrow \operatorname{SK}_{1}\left(U_{\eta}\right) / \ell^{\nu}\right)=\operatorname{im}\left(\operatorname{SK}_{1}\left(Z_{\eta}\right) / \ell^{\nu}\right)
$$

for almost all $\ell \in \mathbb{L}$ and every $\nu \geqslant 1$.

- We now show that for almost all $\ell \in \mathbb{L}$ and every $\nu \geqslant 1$ we even have

$$
\operatorname{ker} \rho_{\ell^{\nu}}^{X} \subseteq \operatorname{im}\left(V\left(Z_{\eta}\right) / \ell^{\nu}\right)
$$

where $V\left(Z_{\eta}\right)$ is the kernel of the norm map. If $\ell$ does not divide \# coker $\left(N_{Z_{\eta} \mid K}\right)$, which is finite by 3.4 .2 , we get the commutative diagram with exact rows

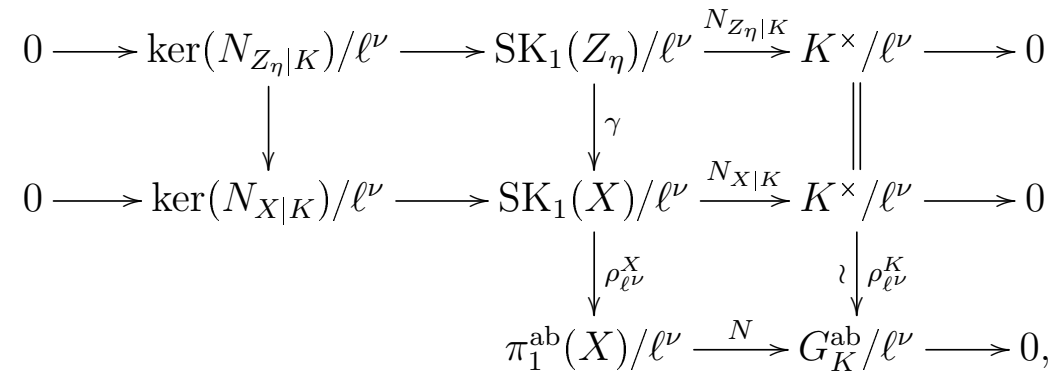


where $\rho_{\ell^{\nu}}^{K}$ is an isomorphism by local class field theory. So if $y \in \operatorname{SK}_{1}\left(Z_{\eta}\right)$ is a preimage of $x \in \operatorname{ker} \rho_{\ell^{\nu}}^{X}$, then it follows that $N \circ \rho_{\ell^{\nu}}^{X} \circ \gamma(y)=0$. By this diagram it follows that $N_{Z_{\eta} \mid K}(y)=0$, hence the claim.

- Let $\mathbb{L}^{\prime} \subseteq \mathbb{L}$ is the set of prime numbers where the finite number of primes from above are removed, then by induction $V\left(Z_{\eta}\right)$ is the direct sum of a finite (!) group $T$ and a $\mathbb{L}^{\prime}$-divisible group $D$. Note that we do not have to assume the condition on $p=\operatorname{char}(k)$ for $Z$, because we removed it from $\mathbb{L}^{\prime}$. So if $\ell \in \mathbb{L}^{\prime}$ does not divide $\# T$, then $V\left(Z_{\eta}\right)$ is $\ell$-divisible and therefore $V\left(Z_{\eta}\right) / \ell^{\nu}$ vanishes. Since ker $\rho_{\ell^{\nu}}^{X}$ lies in the image of $V\left(Z_{\eta}\right) / \ell^{\nu}$ it also vanishes. Therefore $\operatorname{ker} \rho_{\ell^{\nu}}^{X}$ vanishes for almost all $\ell \in \mathbb{L}$ and every $\nu \geqslant 1$.

- It also follows that for almost all $\ell \in \mathbb{L}$ we have that

$$
\operatorname{ker} \rho_{\{\ell\}}^{X}=\lim _{\nu \geqslant 1} \operatorname{ker} \rho_{\ell^{\nu}}^{X}=0 .
$$

- Since by $3.4 .5 \operatorname{ker} \rho^{X}$ is the direct sum of a divisible group and torsion group, it is also the direct sum of a $\mathbb{L}$-divisible group and a $\mathbb{L}$-torsion group by 3.2 .13 and we get by 3.2.11.

$$
\operatorname{ker} \rho^{X} \cong\left(\operatorname{ker} \rho^{X}\right)_{\mathbb{L} \text {-Div }} \oplus \bigoplus_{\ell \in \mathbb{L}}\left(\operatorname{ker} \rho^{X} /\left(\operatorname{ker} \rho^{X}\right)_{\mathbb{L} \text {-Div }}\right)_{\ell \text {-tors }}
$$

and therefore

$$
\left(\operatorname{ker} \rho^{X}\right)_{\mathbb{L}} \cong \prod_{\ell \in \mathbb{L}}\left(\operatorname{ker} \rho^{X}\right)_{\{\ell\}}
$$

Since

$$
\left(\operatorname{ker} \rho^{X}\right)_{\mathbb{L}^{\prime}}=\operatorname{ker} \rho_{\mathbb{L}^{\prime}}^{X}
$$

by 3.4 .5 for every set of prime numbers $\mathbb{L}^{\prime}$, we have by the previous points that $\left(\operatorname{ker} \rho^{X}\right)_{\{\ell\}}=0$ for almost all $\ell \in \mathbb{L}$ and we get

$$
\operatorname{ker} \rho_{\mathbb{L}}^{X} \cong \prod_{\ell \in \mathbb{L}^{\prime}}\left(\operatorname{ker} \rho^{X}\right)_{\{\ell\}} \cong \operatorname{ker} \rho_{\mathbb{L}^{\prime}}^{X},
$$

where $\mathbb{L}^{\prime}$ is only a finite (!) set of prime numbers.

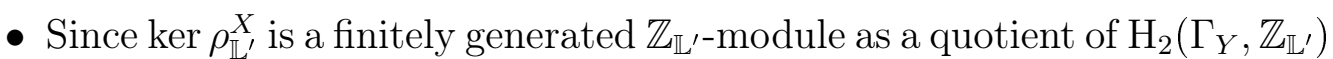
it only has a finite torsion subgroup, because $\mathbb{L}^{\prime}$ is finite. Again with the fact that $\operatorname{ker} \rho_{\mathbb{L}^{\prime}}^{X}=\left(\operatorname{ker} \rho^{X}\right)_{\mathbb{L}^{\prime}}$ and that $\operatorname{ker} \rho^{X}$ is the direct sum of a divisible group and a torsion group it follows from 3.2.14 that $\operatorname{ker} \rho_{\mathbb{L}^{\prime}}^{X}$ is finite. With $\operatorname{ker} \rho_{\mathbb{L}^{\prime}}^{X}=\operatorname{ker} \rho_{\mathbb{L}}^{X}$ also $\operatorname{ker} \rho_{\mathbb{L}}^{X}$ is finite and 3.2.14 again gives us that

$$
\operatorname{ker} \rho^{X} \cong \operatorname{ker} \rho_{\mathbb{L}}^{X} \oplus\left(\operatorname{ker} \rho^{X}\right)_{\mathbb{L} \text {-Div }}
$$

is the direct sum of the finite $\mathbb{L}$-torsion group $\operatorname{ker} \rho_{\mathbb{L}}^{X}$ and the $\mathbb{L}$-divisible $\operatorname{group}\left(\operatorname{ker} \rho^{X}\right)_{\mathbb{L}-\text { Div }}$. 
- We also mention the following: With the three exact sequences of finite groups

$$
\begin{gathered}
\operatorname{ker} \rho_{n}^{X c} \longrightarrow \operatorname{SK}_{1}(X) / n \longrightarrow \pi_{1}^{\mathrm{ab}}(X) / n \longrightarrow 0, \\
{ }_{n}\left(\operatorname{coker} \rho^{X}\right) \longrightarrow\left(\operatorname{im} \rho^{X}\right) / n \longrightarrow \pi_{1}^{\mathrm{ab}}(X) / n \longrightarrow\left(\operatorname{coker} \rho_{n}^{X} \longrightarrow 0,\right. \\
\left.\left(\operatorname{ker} \rho^{X}\right) / n \longrightarrow \rho^{X}\right) / n \longrightarrow \operatorname{SK}_{1}(X) / n \longrightarrow\left(\operatorname{im} \rho^{X}\right) / n \longrightarrow 0,
\end{gathered}
$$

and with coker $\rho_{n}^{X}=\left(\operatorname{coker} \rho^{X}\right) / n$ follows that

$$
\begin{aligned}
\# \operatorname{ker} \rho_{n}^{X} & =\#\left(\operatorname{SK}_{1}(X) / n\right) \cdot \#\left(\operatorname{coker} \rho_{n}^{X}\right) / \#\left(\pi_{1}^{\mathrm{ab}}(X) / n\right) \\
& \leqslant \#\left(\operatorname{SK}_{1}(X) / n\right) \cdot \#\left({ }_{n}\left(\operatorname{coker} \rho^{X}\right)\right) / \#\left(\left(\operatorname{im} \rho^{X}\right) / n\right) \\
& \leqslant \#\left(\left(\operatorname{ker} \rho^{X}\right) / n\right) \cdot \#\left({ }_{n}\left(\operatorname{coker} \rho^{X}\right)\right) \\
& \leqslant \# \operatorname{ker} \rho_{\mathbb{L}}^{X} \cdot \#\left(\operatorname{coker} \rho^{X}\right)_{\text {tors }}
\end{aligned}
$$

is bounded with respect to $n \in \mathbb{N}(\mathbb{L})$. Note, that the both last groups are finite and independent of $n$.

4. By $\operatorname{char}(K)=0$ and the condition on $p$ we can choose $\mathbb{L}$ to be the set of all primes. Consider the commutative diagram

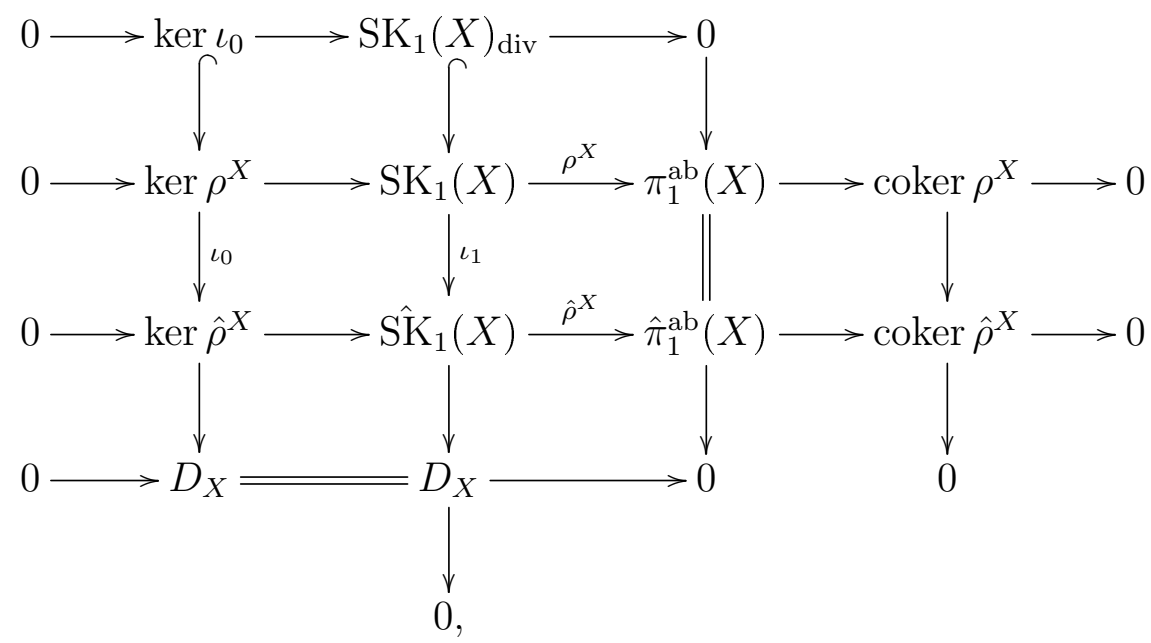

where $D_{X}:=\operatorname{coker}\left(\mathrm{SK}_{1}(X) \stackrel{\iota_{1}}{\rightarrow} \hat{S K}_{1}(X)\right)$. The isomorphism $\pi_{1}^{\mathrm{ab}}(X)=\hat{\pi}_{1}^{\mathrm{ab}}(X)$ induces the equality $\left(\operatorname{ker} \rho^{X}\right)_{\operatorname{div}}=\operatorname{SK}_{1}(X)_{\operatorname{div}}$. So if we mod out these groups on both sides, the snake lemma yields the exact sequence

$$
0 \rightarrow \operatorname{ker} \rho^{X} / \operatorname{SK}_{1}(X)_{\operatorname{div}} \rightarrow \operatorname{ker} \hat{\rho}^{X} \rightarrow D_{X} \rightarrow \operatorname{coker} \rho^{X} \rightarrow \operatorname{coker} \hat{\rho}^{X} \rightarrow 0
$$


Since $\operatorname{SK}_{1}(X) / n$ is finite by the first point and $\operatorname{ker} \rho^{X} / n$ lies in the exact sequence

$$
{ }_{n} \operatorname{im}\left(\rho^{X}\right) \rightarrow \operatorname{ker} \rho^{X} / n \rightarrow \mathrm{SK}_{1}(X) / n,
$$

where ${ }_{n} \operatorname{im}\left(\rho^{X}\right)$ and $\operatorname{ker} \rho^{X} / n$ are also finite. Furthermore, we have that $\left(\operatorname{ker} \rho^{X}\right)^{\wedge}=\operatorname{ker} \hat{\rho}^{X}$ by 3.4 .5 and by the second point that ker $\hat{\rho}^{X}$ is a finitely generated $\hat{\mathbb{Z}}$-module. Since $\pi_{1}^{\mathrm{ab}}(X)$ is also a finitely generated $\hat{\mathbb{Z}}$-module, $\mathrm{SK}_{1}(X)$ also is. By 3.2.15 we get that $D_{X}$ is an uniquely divisible group, since every $\ell$-torsion of a finitely generated $\hat{\mathbb{Z}}$-module is finite. By the last point we have that ker $\hat{\rho}^{X}$ is finite and with 3.2 .14 we see that $\iota_{0}$ is surjective. Since $D_{X}$ is uniquely divisible the exact sequence

$$
0 \rightarrow D_{X} \rightarrow \operatorname{coker} \rho^{X} \rightarrow \operatorname{coker} \hat{\rho}^{X} \rightarrow 0
$$

therfore splits. By the second point we have coker $\hat{\rho}^{X} \cong \mathrm{H}_{1}\left(\Gamma_{Y}, \hat{\mathbb{Z}}\right)$. Since $\left(\operatorname{ker} \rho^{X}\right)$ is of finite exponent we get with 3.2.15 and 3.4.5 that

$$
\left(\operatorname{ker} \rho^{X}\right)_{\operatorname{div}}=\left(\operatorname{ker} \rho^{X}\right)_{\text {Div }}=\operatorname{SK}_{1}(X)_{\text {Div }} \text {, }
$$

and

$$
\operatorname{ker} \rho^{X}=\left(\operatorname{ker} \rho^{X}\right) \oplus \operatorname{SK}_{1}(X)_{\text {Div }}
$$

Corollary 3.4.8. Let $K$ be the field of fractions of an excellent henselian discrete valuation ring $A$ with finite residue field $k$, e.g. $K$ a local field. Let $X$ be a proper and smooth variety over $K$ and let $\mathbb{L}$ be a set of prime numbers not containing $\operatorname{char}(k)$. Then the kernel of the reciprocity map $\operatorname{ker} \rho^{X}$ is the direct sum of a finite group and $a \mathbb{L}$-divisible group. If $X$, furthermore, is geometrically connected over $K$, then also the kernel of the norm map $V(X)$ is the direct sum of a finite group and a $\mathbb{L}$-divisible group.

Proof. By 3.3.3 there is a de Jong alteration $g: \mathfrak{X}^{\prime} \rightarrow \mathfrak{X}$ and where $X^{\prime}=\mathfrak{X}_{\eta}^{\prime}$ is smooth projective and where $Y_{\text {red }}^{\prime}$ is a simple normal crossing variety, $Y^{\prime}:=\mathfrak{X}_{s}^{\prime}$. So $\mathfrak{X}^{\prime}$ satisfies the condition on 3.4.7. Note, that $Y^{\prime}$ and $Y_{\text {red }}^{\prime}$ have the same niveau spectral sequence and homology groups. Let $m$ be the degree of $g$. By [JS03] Lem. 7.4 the cokernel of

$$
g_{*}: \mathrm{H}_{a}^{K}\left(X^{\prime} \mid K, \mathbb{Z} / n(-1)\right) \rightarrow \mathrm{H}_{a}^{K}(X \mid K, \mathbb{Z} / n(-1))
$$

is of exponent $m$ for every integer $n$ not divided by $\operatorname{char}(K)$ by an analogue argument like in 3.4.2. So if we choose $n=\ell^{\nu}$ with $\ell \in \mathbb{L}$ not dividing $m$ the map 
$g_{*}$ is surjective, since $\mathrm{H}_{a}^{K}(X \mid K, \mathbb{Z} / n(-1))$ is $\ell$-torsion. So we get the commutative diagram

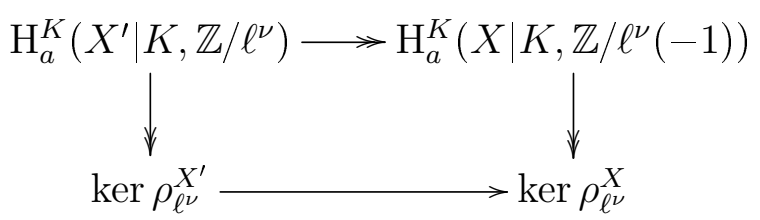

and therefore a surjection

$$
\operatorname{ker} \rho_{\ell^{\nu}}^{X^{\prime}} \rightarrow \operatorname{ker} \rho_{\ell^{\nu}}^{X}
$$

for every prime $\ell \in \mathbb{L}$ not dividing $m$ and every $\nu \geqslant 1$. Because \# $\operatorname{ker} \rho_{\ell^{\nu}}^{X^{\prime}}$ is uniformly bounded by the proof of 3.4.7, $\operatorname{ker} \rho_{\ell^{\nu}}^{X}$ also is with respect to these integers. (Indeed, they vanish for almost all $\ell$.) It only remains the finite number of primes $\ell \mid m$. But for these primes we can find an $\ell^{\prime}$-alteration by 3.3 .3 of degree $m^{\prime}$ prime to $\ell$ and using the same argument as before. So we get that \# ker $\rho_{n}^{X}$ is uniformly bounded with respect to $n \in \mathbb{N}(\mathbb{L})$. So $\operatorname{ker} \rho_{\mathbb{L}}^{X}$ is finite and 3.2 .14 again gives us the claim, since $\operatorname{ker} \rho_{\mathbb{L}}^{X}=\left(\operatorname{ker} \rho^{X}\right)_{\mathbb{L}}$ and $\operatorname{ker} \rho^{X}$ is the direct sum of a torsion group and a divisible group by 3.4.5. Since $\operatorname{ker} \rho^{X} \subseteq V(X)$ is of finite index by 3.4.5, the same holds for $V(X)$ by 3.2 .12 .

Remark 3.4.9. In the setting of 3.4 .8 we had to exclude $p=\operatorname{char}(k)$ from $\mathbb{L}$ even if $\operatorname{char}(K)=0$, in contrast to the $\operatorname{dim} \leqslant 2$ case 3.4.6. This is because we had to deal with two problems:

- The alteration theorem only works outside the prime p.

- We had to assume the Kato conjecture for $\mathrm{H}_{4}^{K}\left(\mathfrak{X} \mid S, \mathbb{Q}_{p} / \mathbb{Z}_{p}(-1)\right)$, which is not proven for $p$ yet. For a relative surface this group clearly vanishes by a dimension argument, so that problem did not occure in this case.

To avoid these problems we would have to show that the p-completion of $V(X)$ is finite, or, equivalently, that $\operatorname{ker} \rho_{p^{\nu}}^{X}$ is bounded with respect to $\nu \geqslant 1$. This was shown in [JS03] for surfaces also using a dimension argument.

\subsection{The kernel of the reciprocity map and its reduction}

In this section we want to discuss if the kernel of the reciprocity map modulo $n$ of a smooth and projective variety over a local field with strict semi-stable reduction injects into the corresponding kernel of its reduction. 
Proposition 3.5.1. Let $d^{\prime} \geqslant 2$ be a fixed integer. If, with the conditions in 3.4.7, we have canonical isomorphisms

$$
\operatorname{ker} \rho_{n}^{X} \cong \operatorname{ker} \rho_{n}^{Y}
$$

for all projective schemes $\mathfrak{X}$ like in 3.4 .7 of relative dimension $d^{\prime}$, then it holds in every dimension $\geqslant d^{\prime}$.

Proof. Let the notions be like in 3.4 .7 and let $\mathfrak{X}$ be projective of relative dimension $d \geqslant d^{\prime}+1 \geqslant 3$. Consider the big diagram from 2.5.6, where $\mathrm{H}_{a}\left({ }_{-}\right)=$ $\mathrm{H}_{a}\left({ }_{-} \mid S, \mathbb{Z} / n(-1)\right)$ :

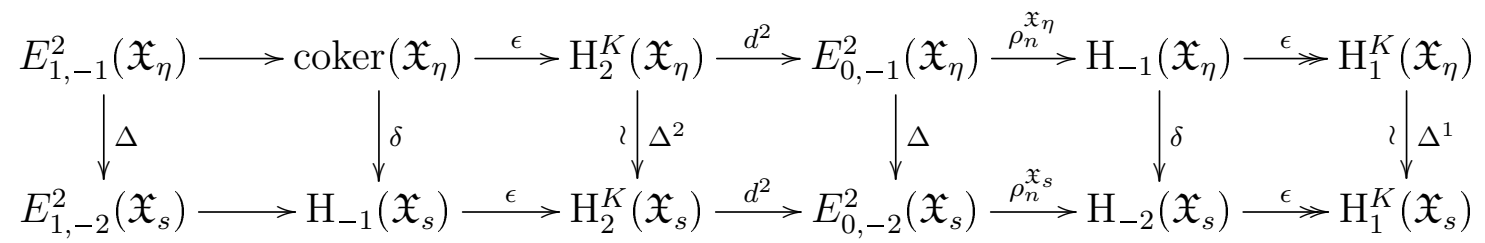

where $\operatorname{coker}\left(\mathfrak{X}_{\eta}\right)=\operatorname{coker}\left(E_{0,0}^{2}\left(\mathfrak{X}_{\eta}\right) \rightarrow \mathrm{H}_{0}\left(\mathfrak{X}_{\eta}\right)\right)$. The isomorphism $\Delta^{2}$ induces a surjection

$$
\operatorname{sp}_{n}: \operatorname{ker} \rho_{n}^{\mathfrak{X}_{\eta}} \rightarrow \operatorname{ker} \rho_{n}^{\mathfrak{X}_{s}} .
$$

And by definition we have

$$
\operatorname{ker} \operatorname{sp}_{n}=\operatorname{ker}\left(E_{0,-1}^{2}\left(\mathfrak{X}_{\eta}\right) \stackrel{\rho_{n}^{\mathfrak{X}_{\eta}} \oplus \Delta}{\longrightarrow} \mathrm{H}_{-1}\left(\mathfrak{X}_{\eta}\right) \oplus E_{0,-2}^{2}\left(\mathfrak{X}_{s}\right)\right) .
$$

From 3.1 .5 we get an exact sequence

$$
\mathrm{H}_{0}\left(\mathfrak{X}_{\eta}\right) \oplus E_{1,-2}^{2}\left(\mathfrak{X}_{s}\right) \longrightarrow \mathrm{H}_{-1}\left(\mathfrak{X}_{s}\right) \longrightarrow E_{0,-1}^{2}\left(\mathfrak{X}_{\eta}\right) \longrightarrow \mathrm{H}_{-1}\left(\mathfrak{X}_{\eta}\right) \oplus E_{0,-2}^{2}\left(\mathfrak{X}_{s}\right) .
$$

So ker $\mathrm{sp}_{n}$ vanishes, if and only if the first map is surjective. By induction on the dimension we will reduce this problem to $d=d^{\prime} \geqslant 2$ :

By 3.3.2 there is a "good divisor" $Z \subseteq \mathfrak{X}$, which then is a projective, flat, regular scheme of relative dimension $d-1 \geqslant d^{\prime} \geqslant 2$ with smooth generic fibre and strict semi-stable reduction. Let $U=\mathfrak{X} \backslash Z$ be the open complement. We get a commutative diagram of exact sequences

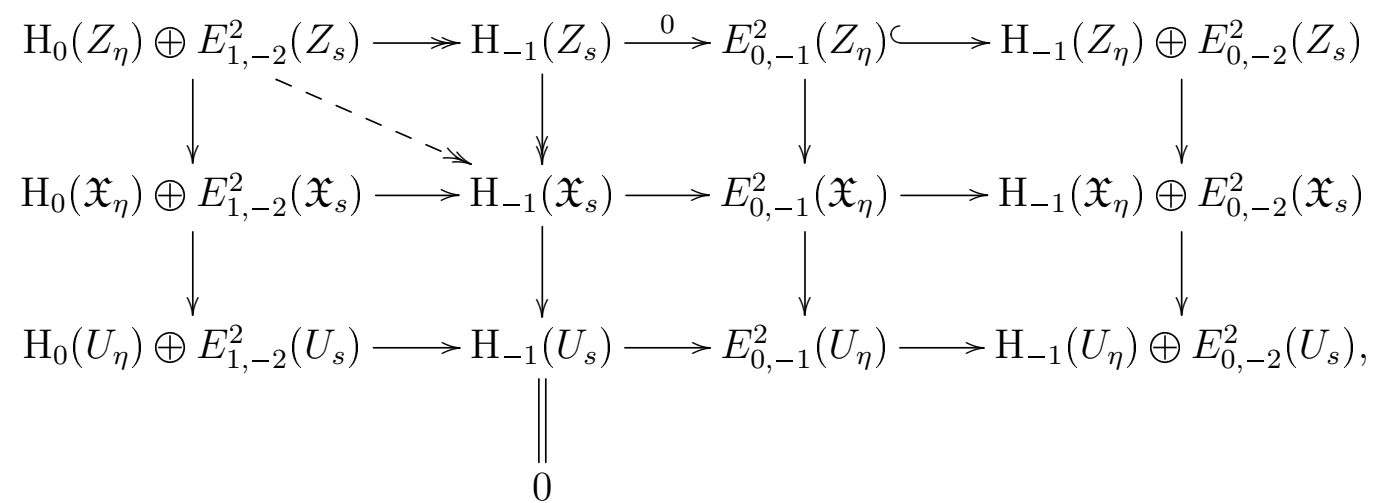


where the zero map in the first line comes by induction and where $\mathrm{H}_{-1}\left(U_{s}\right)$ vanishes by 3.3 .2 because $-1=2-3$ and $2<d^{\prime}+1 \leqslant d$ by assumption on $d$. So the dotted line is surjective and, therefore, the first map in the middle line, too. This implies the statement.

Remark 3.5.2. 1. The assumption of 3.5.1 on the kernels of the reciprocity maps is always true for dimension 0,1 . Unfortunately, the induction step using the vanishing result for the open complement of a good divisor from 3.3.2 ends at relative dimension 2. So the statement can not be reduced to relative curves or points. So the question arises if the two kernels are always isomorphic.

2. If there exists an counterexample in a dimension $\tilde{d}$, then by 3.5 .1 there also will be a counterexample $\mathfrak{X}$ in relative dimension 2 . And $\mathfrak{X} \times_{A} \mathbb{P}_{A}^{\otimes r}$ is then a good candidate for a counterexample in relative dimension $r+2$.

3. To get a criterion for a variety to be a counterexample of 3.5.1 consider the following: If we have

$$
\# \mathrm{H}_{0}\left(\mathfrak{X}_{\eta}\right) * \# E_{1,-2}^{2}\left(\mathfrak{X}_{s}\right)<\# \mathrm{H}_{-1}\left(\mathfrak{X}_{s}\right)
$$

then the map

$$
\mathrm{H}_{0}\left(\mathfrak{X}_{\eta}\right) \oplus E_{1,-2}^{2}\left(\mathfrak{X}_{s}\right) \longrightarrow \mathrm{H}_{-1}\left(\mathfrak{X}_{s}\right)
$$

can not be surjective and therefore the kernels of the reciprocity maps modulo $n$ must differ. So the task is to estimate the three terms. Put $X=\mathfrak{X}_{\eta}$ and $Y=\mathfrak{X}_{s}$. And let $n$ be an integer not divided by the residue characteristic $p=\operatorname{char}(k)$.

a) $B y$ 2.5.3 we have

$$
E_{1,-2}^{2}(Y \mid S, \mathbb{Z} / n(-1)) \cong E_{1,0}^{2}(Y \mid k, \mathbb{Z} / n(0))
$$

And by 2.2.4 there is a first quadrant spectral sequence of homological type

$$
{ }^{I} \tilde{E}_{r, t}^{1}=E_{t, 0}^{2}\left(Y^{[r]}\right) \Longrightarrow \tilde{H}_{r+t}(Y)=E_{r+t, 0}^{2}(Y),
$$

leading to the exact sequence:

$$
{ }^{I} \tilde{E}_{0,1}^{2} \longrightarrow E_{1,0}^{2}(Y) \longrightarrow{ }^{I} \tilde{E}_{1,0}^{2} \longrightarrow 0
$$


With 3.1.4 for smooth varieties we get:

$$
\begin{aligned}
{ }^{I} \tilde{E}_{0,1}^{2} & =\operatorname{coker}\left({ }^{I} \tilde{E}_{0,1}^{1} \leftarrow{ }^{I} \tilde{E}_{1,1}^{1}\right) \\
& =\operatorname{coker}\left(E_{0,1}^{2}\left(Y^{[0]}\right) \leftarrow E_{0,1}^{2}\left(Y^{[1]}\right)\right) \\
& \cong \operatorname{coker}\left(\mathrm{H}_{1}\left(Y^{[0]} \mid k, \mathbb{Z} / n\right) \leftarrow \mathrm{H}_{1}\left(Y^{[1]} \mid k, \mathbb{Z} / n\right)\right) \\
& \cong \operatorname{coker}\left(\mathrm{H}^{2 d-1}\left(Y^{[0]}, \mathbb{Z} / n(d)\right) \leftarrow \mathrm{H}^{2 d-3}\left(Y^{[1]}, \mathbb{Z} / n(d-1)\right)\right) \\
& \cong \operatorname{ker}\left(\mathrm{H}^{2}\left(Y^{[0]}, \mathbb{Z} / n\right) \rightarrow \mathrm{H}^{2}\left(Y^{[1]}, \mathbb{Z} / n\right)\right)^{\vee}, \\
{ }^{I} \tilde{E}_{1,0}^{2} & =\mathrm{H}\left({ }^{I} \tilde{E}_{0,0}^{1} \leftarrow{ }^{I} \tilde{E}_{1,0}^{1} \leftarrow{ }^{I} \tilde{E}_{2,0}^{1}\right) \\
& =\mathrm{H}\left(E_{0,0}^{2}\left(Y^{[0]}\right) \leftarrow E_{0,0}^{2}\left(Y^{[1]}\right) \leftarrow E_{0,0}^{2}\left(Y^{[2]}\right)\right) \\
& \cong \mathrm{H}\left(\mathrm{CH}_{0}\left(Y^{[0]}\right) / n \leftarrow \mathrm{CH}_{0}\left(Y^{[1]}\right) / n \leftarrow \mathrm{CH}_{0}\left(Y^{[2]}\right) / n .\right)
\end{aligned}
$$

So we get inequalities

$$
\begin{aligned}
\left.\# E_{1,0}^{2}(Y \mid k, \mathbb{Z} / n)\right) & \leqslant \#^{I} \tilde{E}_{0,1}^{2} * \#^{I} \tilde{E}_{1,0}^{2} \\
& \leqslant \# \mathrm{H}^{2}\left(Y^{[0]}, \mathbb{Z} / n\right) * \# \mathrm{H}_{1}\left(\mathrm{CH}_{0}\left(Y^{[\bullet]}\right) / n\right) .
\end{aligned}
$$

Furthermore, let $\Gamma=\operatorname{Gal}(\bar{k} \mid k) \cong \hat{\mathbb{Z}}$ be the absolute Galois group of $k$ and $\bar{Z}$ be the base change of a $k$-variety $Z$ with $\bar{k}$, then the Hochschild-Serre spectral sequence

$$
\tilde{E}_{2}^{p, q}=\mathrm{H}^{p}\left(\Gamma, \mathrm{H}^{q}(\bar{Z}), \bar{F}\right) \Longrightarrow \mathrm{H}^{p+q}(Z, F)
$$

gives short exact sequences

$$
0 \rightarrow \mathrm{H}^{m-1}(\bar{Z}, \bar{F})_{\Gamma} \rightarrow \mathrm{H}^{m}(Z, F) \rightarrow \mathrm{H}^{m}(\bar{Z}, \bar{F})^{\Gamma} \rightarrow 0 .
$$

So we get

$$
\# \mathrm{H}^{2}\left(Y^{[0]}, \mathbb{Z} / n\right)=\# \mathrm{H}^{2}\left(\bar{Y}^{[0]}, \mathbb{Z} / n\right)^{\Gamma} * \# \mathrm{H}^{1}\left(\bar{Y}^{[0]}, \mathbb{Z} / n\right)_{\Gamma} .
$$

b) $B y$ 2.5.3 and 3.1.3 we get

$$
\mathrm{H}_{-1}(Y \mid S, \mathbb{Z} / n(-1)) \cong \mathrm{H}_{1}(Y \mid k, \mathbb{Z} / n(0)) \cong \mathrm{H}^{2}(Y, \mathbb{Z} / n)^{\vee} .
$$

And by 2.4.6 there is a first quadrant spectral sequence of cohomological type

$$
{ }^{I} E_{1}^{r, q}=\mathrm{H}^{q}\left(Y^{[r]}, \mathbb{Z} / n\right) \Longrightarrow{ }^{I} E^{r+q}=\mathrm{H}^{r+q}(Y, \mathbb{Z} / n),
$$

leading to the exact sequence

$$
{ }^{I} E_{2}^{0,1} \longrightarrow{ }^{I} E_{2}^{2,0} \longrightarrow \operatorname{ker}\left({ }^{I} E^{2} \rightarrow{ }^{I} E_{2}^{0,2}\right) \longrightarrow{ }^{I} E_{2}^{1,1} \longrightarrow{ }^{I} E_{2}^{3,0},
$$


where we have

$$
\begin{aligned}
{ }^{I} E_{2}^{0,1} & =\operatorname{ker}\left({ }^{I} E_{1}^{0,1} \rightarrow{ }^{I} E_{1}^{1,1}\right) \\
& =\operatorname{ker}\left(\mathrm{H}^{1}\left(Y^{[0]}, \mathbb{Z} / n\right) \rightarrow \mathrm{H}^{1}\left(Y^{[1]}, \mathbb{Z} / n\right)\right) \\
& \cong \operatorname{coker}\left(\pi_{1}^{\mathrm{ab}}\left(Y^{[0]}\right) / n \leftarrow \pi_{1}^{\mathrm{ab}}\left(Y^{[1]}\right) / n\right)^{\vee} \\
& \cong \operatorname{coker}\left(\mathrm{CH}_{0}\left(Y^{[0]}\right) / n \leftarrow \mathrm{CH}_{0}\left(Y^{[1]}\right) / n\right)^{\vee} \\
& \cong\left(\mathrm{CH}_{0}(Y) / n\right)^{\vee}, \\
{ }^{I} E_{2}^{2,0} & =\mathrm{H}\left({ }^{I} E_{1}^{1,0} \rightarrow{ }^{I} E_{1}^{2,0} \rightarrow{ }^{I} E_{1}^{3,0}\right) \\
& =\mathrm{H}\left(\mathrm{H}^{0}\left(Y^{[1]}, \mathbb{Z} / n\right) \rightarrow \mathrm{H}^{0}\left(Y^{[2]}, \mathbb{Z} / n\right) \rightarrow \mathrm{H}^{0}\left(Y^{[3]}, \mathbb{Z} / n\right),\right) \\
& \cong \mathrm{H}_{2}\left(\Gamma_{Y}, \mathbb{Z} / n\right)^{\vee}, \\
{ }^{I} E_{2}^{0,2} & \left.\left.=\operatorname{ker}\left({ }^{I} E_{1}^{0,2}\right) \rightarrow{ }^{I} E_{1}^{1,2}\right)\right) \\
& =\operatorname{ker}\left(\mathrm{H}^{2}\left(Y^{[0]}, \mathbb{Z} / n\right) \rightarrow \mathrm{H}^{2}\left(Y^{[1]}, \mathbb{Z} / n\right)\right), \\
{ }^{I} E_{2}^{1,1} & =\mathrm{H}\left({ }^{I} E_{1}^{0,1} \rightarrow{ }^{I} E_{1}^{1,1} \rightarrow{ }^{I} E_{1}^{2,1}\right) \\
& =\mathrm{H}\left(\mathrm{H}^{1}\left(Y^{[0]}, \mathbb{Z} / n\right) \rightarrow \mathrm{H}^{1}\left(Y^{[1]}, \mathbb{Z} / n\right) \rightarrow \mathrm{H}^{1}\left(Y^{[2]}, \mathbb{Z} / n\right)\right) \\
& =\mathrm{H}\left(\pi_{1}^{\mathrm{ab}}\left(Y^{[0]}\right) / n \leftarrow \pi_{1}^{\mathrm{ab}}\left(Y^{[1]}\right) / n \leftarrow \pi_{1}^{\mathrm{ab}}\left(Y^{[2]}\right) / n\right)^{\vee} \\
& =\mathrm{H}\left(\mathrm{CH}_{0}\left(Y^{[0]}\right) / n \leftarrow \mathrm{CH}_{0}\left(Y^{[1]}\right) / n \leftarrow \mathrm{CH}_{0}\left(Y^{[2]}\right) / n\right)^{\vee}, \\
& \\
{ }^{I} E_{2}^{3,0} & =\mathrm{H}\left({ }^{I} E_{1}^{2,0} \rightarrow{ }^{I} E_{1}^{3,0} \rightarrow{ }^{I} E_{1}^{4,0}\right) \\
& =\mathrm{H}\left(\mathrm{H}^{0}\left(Y^{[2]}, \mathbb{Z} / n\right) \rightarrow \mathrm{H}^{0}\left(Y^{[3]}, \mathbb{Z} / n\right) \rightarrow \mathrm{H}^{0}\left(Y^{[4]}, \mathbb{Z} / n\right),\right) \\
& \cong \mathrm{H}{ }_{3}\left(\Gamma_{Y}, \mathbb{Z} / n\right)^{\vee} .
\end{aligned}
$$

So we get inequalities:

$$
\begin{aligned}
& \# \mathrm{H}^{1}\left(Y^{[0]}, \mathbb{Z} / n\right) * \# \mathrm{H}^{2}(Y, \mathbb{Z} / n) * \# \mathrm{H}_{3}\left(\Gamma_{Y}, \mathbb{Z} / n\right) \\
\geqslant & \#^{I} E_{2}^{0,1} * \#^{I} E^{2} * \#^{I} E_{2}^{3,0} \\
\geqslant & \#^{I} E_{2}^{0,1} * \# \operatorname{ker}\left({ }^{I} E^{2} \rightarrow{ }^{I} E_{2}^{0,2}\right) * \#^{I} E_{2}^{3,0} \\
\geqslant & \#^{I} E_{2}^{2,0} * \# E^{I} E_{2}^{1,1} \\
= & \# \mathrm{H}_{2}\left(\Gamma_{Y}, \mathbb{Z} / n\right) * \# \mathrm{H}_{1}\left(\mathrm{CH}_{0}\left(Y^{[\bullet]}\right) / n\right) .
\end{aligned}
$$

And the Hochschild-Serre spectral sequence again gives:

$$
\# \mathrm{H}^{1}\left(Y^{[0]}, \mathbb{Z} / n\right)=\# \mathrm{H}^{0}\left(\bar{Y}^{[0]}, \mathbb{Z} / n\right)_{\Gamma} * \# \mathrm{H}^{1}\left(\bar{Y}^{[0]}, \mathbb{Z} / n\right)^{\Gamma} .
$$

c) By 2.5.3 and 3.1.3 and 2.4.3 we get

$$
\begin{aligned}
\mathrm{H}_{0}(X \mid S, \mathbb{Z} / n(-1)) & \cong \mathrm{H}_{0}(X \mid K, \mathbb{Z} / n(-1)) \\
& \cong \mathrm{H}^{2 d}(X, \mathbb{Z} / n(d+1)) \\
& \cong \mathrm{H}^{2}(X, \mathbb{Z} / n)^{\vee} .
\end{aligned}
$$

Now, let $G=\operatorname{Gal}(\bar{K} \mid K)$ be the absolute Galois group of $K, I \subseteq G$ its inertia subgroup, and $\Gamma \cong \hat{\mathbb{Z}}$ the factor group corresponding to the 
maximal unramified extension $\tilde{K}$ of $K$. For a $K$-variety $Z$, let $\bar{Z}, \tilde{Z}$ resp., be the base change with $\bar{K}, \tilde{K}$ resp.. By [NSW08] Thm 7.1.8. we have $\operatorname{cd}_{n} I \leqslant 1$ and also $\operatorname{cd}_{n} \Gamma \leqslant 1$. So the Hochschild-Serre spectral sequence yields the short exact sequences

$$
\begin{gathered}
0 \longrightarrow \mathrm{H}^{1}(\tilde{X}, \mathbb{Z} / n)_{\Gamma} \longrightarrow \mathrm{H}^{2}(X, \mathbb{Z} / n) \longrightarrow \mathrm{H}^{2}(\tilde{X}, \mathbb{Z} / n)^{\Gamma} \longrightarrow 0 \\
0 \longrightarrow \mathrm{H}^{1}(\bar{X}, \mathbb{Z} / n)_{I} \longrightarrow \mathrm{H}^{2}(\tilde{X}, \mathbb{Z} / n) \longrightarrow \mathrm{H}^{2}(\bar{X}, \mathbb{Z} / n)^{I} \longrightarrow 0 \\
0 \longrightarrow \mathrm{H}^{0}(\bar{X}, \mathbb{Z} / n)_{I} \longrightarrow \mathrm{H}^{1}(\tilde{X}, \mathbb{Z} / n) \longrightarrow \mathrm{H}^{1}(\bar{X}, \mathbb{Z} / n)^{I} \longrightarrow 0
\end{gathered}
$$

So we get the inequalitiy

$$
\begin{aligned}
\# \mathrm{H}^{2}(X) & =\# \mathrm{H}^{1}(\tilde{X})_{\Gamma} * \# \mathrm{H}^{2}(\tilde{X})^{\Gamma} \\
& \leqslant \#\left(\mathrm{H}^{0}(\bar{X})_{I}\right)_{\Gamma} * \#\left(\mathrm{H}^{1}(\bar{X})^{I}\right)_{\Gamma} * \#\left(\mathrm{H}^{1}(\bar{X})_{I}\right)^{\Gamma} * \#\left(\mathrm{H}^{2}(\bar{X})^{I}\right)^{\Gamma} \\
& =\# \mathrm{H}^{0}(\bar{X})^{G} *\left(\# \mathrm{H}^{1}(\bar{X})^{G}\right)^{2} * \# \mathrm{H}^{2}(\bar{X})^{G},
\end{aligned}
$$

since $n$ is prime to $p$, the inertia subgroup I operates only via its tame quotient, which is a monothetic pro-finite group. So the order of the fix module and cofix module coincide in the equalities above.

Joining the first three points together we get the following statement:

Proposition 3.5.3. If the inequality with $\mathbb{Z} / n$-coefficients

$$
\begin{gathered}
\# \mathrm{H}_{2}\left(\Gamma_{Y}\right)>\# \mathrm{H}_{3}\left(\Gamma_{Y}\right) * \# \mathrm{H}^{0}\left(\bar{Y}^{[0]}\right)^{\Gamma} *\left(\# \mathrm{H}^{1}\left(\bar{Y}^{[0]}\right)^{\Gamma}\right)^{2} * \# \mathrm{H}^{2}\left(\bar{Y}^{[0]}\right)^{\Gamma} \\
* \# \mathrm{H}^{0}(\bar{X})^{G} *\left(\# \mathrm{H}^{1}(\bar{X})^{G}\right)^{2} * \# \mathrm{H}^{2}(\bar{X})^{G}
\end{gathered}
$$

holds, then the surjection $\operatorname{ker} \rho_{n}^{X} \rightarrow \operatorname{ker} \rho_{n}^{Y}$ is not injective.

Remark 3.5.4. If in the setting of 3.5.3 one does not have information over the étale cohomology groups of $\bar{X}$, one can estimate them with the cohomological spectral sequence of vanishing cycles [RZ82], which is $\Gamma$-equivariant:

$$
E_{1}^{s, t}=\bigoplus_{k \geqslant \max (0, s)} \mathrm{H}^{t+2 s-2 k}\left(\bar{Y}^{[2 k-s]}, \mathbb{Z} / n(s-k)\right) \Longrightarrow \mathrm{H}^{s+t}(\bar{X}, \mathbb{Z} / n)^{P}
$$

We can estimate $\mathrm{H}^{s+t}(\bar{X}, \mathbb{Z} / n)^{P}$ by comparing the terms $E_{1}^{s, t}$ with the limit terms 
$E_{\infty}^{s, t}$ which are subquotients:

$$
\begin{array}{ll}
\# \mathrm{H}^{0}(\bar{X}, \mathbb{Z} / n)^{P} & =\# E_{\infty}^{0,0} \leqslant \# \mathrm{H}^{0}\left(\bar{Y}^{[0]}, \mathbb{Z} / n\right), \\
\# F^{1} \mathrm{H}^{1}(\bar{X}, \mathbb{Z} / n)^{P} & =\# E_{\infty}^{1,0} \leqslant \# \mathrm{H}^{0}\left(\bar{Y}^{[1]}, \mathbb{Z} / n\right), \\
\# F^{0} \mathrm{H}^{1}(\bar{X}, \mathbb{Z} / n)^{P} / F^{1} & =\# E_{\infty}^{0,1} \leqslant \# \mathrm{H}^{1}\left(\bar{Y}^{[0]}, \mathbb{Z} / n\right), \\
\# \mathrm{H}^{1}(\bar{X}, \mathbb{Z} / n)^{P} / F^{0} & =\# E_{\infty}^{-1,2} \leqslant \# \mathrm{H}^{0}\left(\bar{Y}^{[1]}, \mathbb{Z} / n(-1)\right), \\
\# F^{2} \mathrm{H}^{2}(\bar{X}, \mathbb{Z} / n)^{P} & =\# E_{\infty}^{2,0} \leqslant \# \mathrm{H}^{0}\left(\bar{Y}^{[2]}, \mathbb{Z} / n\right), \\
\# F^{1} \mathrm{H}^{2}(\bar{X}, \mathbb{Z} / n)^{P} / F^{2} & =\# E_{\infty}^{1,1} \leqslant \# \mathrm{H}^{1}\left(\bar{Y}^{[1]}, \mathbb{Z} / n\right), \\
\# F^{0} \mathrm{H}^{2}(\bar{X}, \mathbb{Z} / n)^{P} / F^{1} & =\# E_{\infty}^{0,2} \leqslant \# \mathrm{H}^{2}\left(\bar{Y}^{[0]}, \mathbb{Z} / n\right) * \# \mathrm{H}^{0}\left(\bar{Y}^{[2]}, \mathbb{Z} / n(-1)\right), \\
\# F^{-1} \mathrm{H}^{2}(\bar{X}, \mathbb{Z} / n)^{P} / F^{0} & =\# E_{\infty}^{-1,3} \leqslant \# \mathrm{H}^{1}\left(\bar{Y}^{[1]}, \mathbb{Z} / n(-1)\right), \\
\# \mathrm{H}^{2}(\bar{X}, \mathbb{Z} / n)^{P} / F^{-1} & =\# E_{\infty}^{-2,4} \leqslant \# \mathrm{H}^{0}\left(\bar{Y}^{[2]}, \mathbb{Z} / n(-2)\right) .
\end{array}
$$

Taking the products, we get inequalities

$$
\begin{aligned}
\# \mathrm{H}^{0}(\bar{X})^{P} \leqslant & \# \mathrm{H}^{0}\left(\bar{Y}^{[0]}\right) \\
\# \mathrm{H}^{1}(\bar{X})^{P} \leqslant & \# \mathrm{H}^{0}\left(\bar{Y}^{[1]}\right) * \# \mathrm{H}^{1}\left(\bar{Y}^{[0]}\right) * \# \mathrm{H}^{0}\left(\bar{Y}^{[1]}\right)(-1) \\
\# \mathrm{H}^{2}(\bar{X})^{P} \leqslant & \# \mathrm{H}^{0}\left(\bar{Y}^{[2]}\right) * \# \mathrm{H}^{1}\left(\bar{Y}^{[1]}\right) * \# \mathrm{H}^{0}\left(\bar{Y}^{[2]}\right)(-1) \\
& * \# \mathrm{H}^{2}\left(\bar{Y}^{[0]}\right) * \# \mathrm{H}^{1}\left(\bar{Y}^{[1]}\right)(-1) * \# \mathrm{H}^{0}\left(\bar{Y}^{[2]}\right)(-2) .
\end{aligned}
$$

Note, that $P$ is a certain subgroup of $I$, and they act trivially on $\mathrm{H}^{s+t}(\bar{X}, \mathbb{Z} / n)$ in the case of simple normal crossings for $\bar{Y}$ by [RZ82] Kor. 2.25.

Lemma 3.5.5. Let $\bar{k}$ be an algebraic closed field and $n$ an integer not divided by $\operatorname{char}(\bar{k})$. Then we have:

1.

$$
\mathrm{H}^{r}\left(\mathbb{A} \frac{d}{k}, \mathbb{Z} / n\right)= \begin{cases}\mathbb{Z} / n & \text { for } r=0 \\ 0 & \text { otherwise }\end{cases}
$$

2.

$$
\mathrm{H}^{r}\left(\mathbb{P}_{\bar{k}}^{d}, \mathbb{Z} / n\right)= \begin{cases}\mathbb{Z} / n\left(-\frac{r}{2}\right) & \text { for even } r, \quad 0 \leqslant r \leqslant 2 d \\ 0 & \text { otherwise. }\end{cases}
$$

3.

$$
\mathrm{H}^{r}\left(\mathbb{P}_{\bar{k}}^{\otimes d}, \mathbb{Z} / n\right)= \begin{cases}\mathbb{Z} / n\left(-\frac{r}{2}\right)^{\left(\frac{d}{2}\right)} & \text { for even } r, \quad 0 \leqslant r \leqslant 2 d \\ 0 & \text { otherwise, }\end{cases}
$$

where $\mathbb{P}_{\bar{k}}^{\otimes d}$ is the $d$-fold product $\mathbb{P}_{\bar{k}}^{1} \times_{\bar{k}} \cdots \times_{\bar{k}} \mathbb{P}_{\bar{k}}^{1}$ of $\mathbb{P}_{\bar{k}}^{1}$. 
4. Let $X=V_{+}(g) \hookrightarrow \mathbb{P}_{\bar{k}}^{d+1}$ be a smooth (!), projective hypersurface definied by a homogeneous polynomial $g$ of degree $f$ and dimension $d$. Then

$$
\mathrm{H}^{r}(X, \mathbb{Z} / n)= \begin{cases}\mathrm{H}^{r}\left(\mathbb{P} \frac{d}{k}, \mathbb{Z} / n\right) & \text { for } r \neq d, \\ \mathrm{H}^{d}\left(\mathbb{P} \frac{d}{k}, \mathbb{Z} / n\right) \oplus \mathrm{H}^{d}(X, \mathbb{Z} / n)^{\prime} & \text { for } r=d,\end{cases}
$$

with the free $\mathbb{Z} / n$-module

$$
\mathrm{H}^{d}(X, \mathbb{Z} / n)^{\prime}:=\operatorname{ker}\left(\mathrm{H}^{d}(X, \mathbb{Z} / n) \stackrel{\text { Gys }}{\longrightarrow} \mathrm{H}^{d+2}\left(\mathbb{P}_{\bar{k}}^{d+1}, \mathbb{Z} / n(1)\right)\right)
$$

of rank

$$
\operatorname{rank}_{\mathbb{Z} / n} \mathrm{H}^{d}(X, \mathbb{Z} / n)^{\prime}=\frac{(f-1)^{d+2}+(-1)^{d}(f-1)}{f} .
$$

5. Let $X \hookrightarrow \mathbb{P}_{\bar{k}}^{\otimes(d+1)}$ be a smooth, projective hypersurface definied by a multihomogeneous polynomial $g$ of dimension $d$. Then

$$
\mathrm{H}^{r}(X, \mathbb{Z} / n)= \begin{cases}\mathrm{H}^{r}\left(\mathbb{P}_{\bar{k}}^{\otimes(d+1)}, \mathbb{Z} / n\right) & \text { for } r \leqslant d-1, \\ \mathrm{H}^{r}\left(\mathbb{P}_{\bar{k}}^{\otimes(d+1)}, \mathbb{Z} / n(1)\right) & \text { for } r \geqslant d+1 .\end{cases}
$$

Proof. See [Mil08] example 16.3 and 16.4. For $\mathbb{P}_{\bar{k}}^{\otimes d}$ use induction on $d$ and the Künneth formula [Mil08] §22. For the last point consider the Gysin sequence [Mil08] $\S 16$ :

$$
\mathrm{H}^{r+1}(U)(1) \rightarrow \mathrm{H}^{r}(X) \rightarrow \mathrm{H}^{r+2}\left(\mathbb{P}_{\bar{k}}^{\otimes(d+1)}\right)(1) \rightarrow \mathrm{H}^{r+2}(U)(1)
$$

where $U$ is the complement of $X$ in $\mathbb{P}_{\bar{k}}^{\otimes(d+1)}$, which is affine and of dimension $d+1$, and for which $\mathrm{H}^{r+1}(U)(1)$ and $\mathrm{H}^{r+2}(U)(1)$ vanish by Mil08] $\S 14$ for $r+1 \geqslant d+2$. For $r \leqslant d-1$ we get by Poincaré duality, cf. Mil08] $\$ 24$ :

$$
\begin{aligned}
\mathrm{H}^{r}(X, \mathbb{Z} / n) & \cong \mathrm{H}^{2 d-r}\left(X, \mathbb{Z} / n^{\vee}(d)\right)^{\vee} \\
& \cong \mathrm{H}^{2 d-r+2}\left(\mathbb{P}_{\bar{k}}^{\otimes(d+1)}, \mathbb{Z} / n(d+1)\right)^{\vee} \\
& \cong \mathrm{H}^{(2 d+2)-(2 d-r+2)}\left(\mathbb{P}_{\bar{k}}^{\otimes(d+1)}, \mathbb{Z} / n(d+1)^{\vee}(d+1)\right)^{\vee} \\
& \cong \mathrm{H}^{r}\left(\mathbb{P}_{\bar{k}}^{\otimes(d+1)}, \mathbb{Z} / n\right),
\end{aligned}
$$

where the second isomorphism is the Gysin isomorphism as before and holds because $2 d-r+1>d+1=\operatorname{dim} U$ for $r \leqslant d-1$.

Remark 3.5.6. Consider the following strategy to construct counterexamples:

- Construct a simple normal crossing variety $\bar{Y}$ consisting of smooth hypersurfaces $\bar{Y}_{i}=V_{+}\left(f_{i}\right)$ in the projective space $\mathbb{P}^{d+1}$ or $\mathbb{P}^{\otimes(d+1)}$, resp., over the algebraic closure $\bar{k}$ of a finite field. 
- Take a finite subfield $k$ of $\bar{k}$ over which all $f_{i}$ are defined and such that every connected component of any $Y^{[r]}$ is geometrically connected, where $Y=$ $V_{+}\left(f_{1} \cdots f_{s}\right)$ is the corresponding variety over $k$.

- The reciprocity map $\rho_{n}^{Y}$ then by 1.5.4 has trivial kernel.

- Construct a lift $\tilde{f}$ of the product $f_{1} \cdots f_{s}$ to a henselian discrete valuation ring A with residue field $k$, such that $V_{+}(\tilde{f})$ is a regular connected subscheme of $\mathbb{P}_{A}^{d+1}$ or $\mathbb{P}_{A}^{\otimes(d+1)}$, resp., with geometrically connected and smooth generic fibre $\bar{X}$ over $\bar{K}$, where $K=\operatorname{Quot}(A)$.

- $\bar{X}$ is then a hypersurface in $\mathbb{P}^{d+1}$ or $\mathbb{P}^{\otimes(d+1)}$ over $\bar{K}$.

- For $d \geqslant 3$ we then by 3.5.5 have

$$
\# \mathrm{H}^{2}(X, \mathbb{Z} / n) \leqslant \# \mathrm{H}^{0}(\bar{X}, \mathbb{Z} / n) *\left(\# \mathrm{H}^{1}(\bar{X}, \mathbb{Z} / n)\right)^{2} * \# \mathrm{H}^{2}(\bar{X}, \mathbb{Z} / n)=n^{2},
$$

$n^{d+2}$, resp., with exponents independent on the degree of $\tilde{f}$ and the number $n$.

- If we constructed $\bar{Y}$ such that $\# \mathrm{H}_{2}\left(\Gamma_{\bar{Y}}, \mathbb{Z} / n\right)>n^{2}$ or $n^{d+2}$, resp., then there must be a non-trivial element in

$$
\operatorname{ker} \rho_{n}^{X} \cong \operatorname{coker}\left(\mathrm{H}^{2}(X, \mathbb{Z} / n)^{\vee} \rightarrow \mathrm{H}_{2}\left(\Gamma_{Y}, \mathbb{Z} / n\right)\right),
$$

whereas $\operatorname{ker} \rho_{n}^{Y}=0$.

This strategy has the problem, that it is in a way independent on n. It would also work, if we took the projective limit of the above groups over $n=\ell^{\nu}$ for a prime

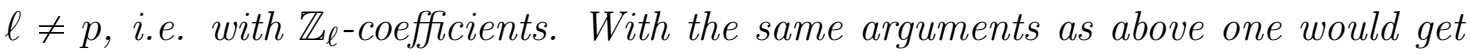
that

$$
\operatorname{rank}_{\mathbb{Z}_{\ell}} \operatorname{ker} \rho_{\{\ell\}}^{X}>0=\operatorname{rank}_{\mathbb{Z}_{\ell}} \operatorname{ker} \rho_{\{\ell\}}^{Y},
$$

which contradicts the fact, that $\rho_{\{\ell\}}^{X}$ is finite by 3.4.8. So this strategy can not work properly. Since it is no problem to construct a huge $\mathrm{H}_{2}\left(\Gamma_{Y}\right)$ with intersecting hypersurfaces, the main problem is to find a regular (!) hypersurface lift $\mathfrak{X}$ of $Y$. The examples 3.5 .8 shows that everything can be done, even that $X$ is smooth, but only the regularity of $\mathfrak{X}$ is missing.

Example 3.5.7 (Cf. [Sat05], [Fri83]). Let $K$ be a local field with ring of integers $A$ and residue field $k$. Let $d \geqslant 3$ and $m \geqslant d+6$ be integers. Fix a monic irreducible polynomial $f \in k[T]$ of degree $m$, let $h=k[T] / f$ be the corresponding field extension and $Q=\operatorname{Spec}(h)$. Furthermore, let $Y$ be the hypersurface in $\mathbb{P}_{k}^{\otimes(d+1)}$ defined by the multi-homogeneous polynomial of degree $(m, m, 2,0, \ldots, 0)$ :

$$
F:=\left(f\left(T_{1} / T_{1}^{\prime}\right) T_{1}^{\prime m}\right) *\left(f\left(T_{2} / T_{2}^{\prime}\right) T_{2}^{\prime m}\right) *\left(T_{3} T_{3}^{\prime}\right),
$$


i.e. we have

$$
Y=\left(\left(Q \times \mathbb{P}_{k}^{1} \times \mathbb{P}_{k}^{1}\right) \cup\left(\mathbb{P}_{k}^{1} \times Q \times \mathbb{P}_{k}^{1}\right) \cup\left(\mathbb{P}_{k}^{1} \times \mathbb{P}_{k}^{1} \times\{O, \infty\}\right)\right) \times \mathbb{P}_{k}^{\otimes(d-2)} .
$$

Let $g, g^{\prime}$ resp., be a separable polynomial in $k[T]$ of degree $m$ or $m-1$, degree 2 resp., which is prime to $f$, prime to $T$ resp.. Furthermore take monic lifts $\tilde{f}, \tilde{g}, \tilde{g}^{\prime}$ of $f, g, g^{\prime}$ in $A[T]$ of the same degrees. Let $\mathfrak{X}$ be the projective subscheme of $\mathbb{P}_{A}^{\otimes(d+1)}$ defined by the multi-homogeneous polynomial of degree $(m, m, 2,0, \ldots, 0)$ :

$$
\tilde{F}+\pi * \tilde{G},
$$

where $\pi$ is a prime element of $A$ and

$$
\begin{aligned}
& \tilde{F}=\left(\tilde{f}\left(T_{1} / T_{1}^{\prime}\right) T_{1}^{\prime m}\right) *\left(\tilde{f}\left(T_{2} / T_{2}^{\prime}\right) T_{2}^{\prime m}\right) *\left(T_{3} T_{3}^{\prime}\right), \\
& \tilde{G}=\left(\tilde{g}\left(T_{1} / T_{1}^{\prime}\right) T_{1}^{\prime m}\right) *\left(\tilde{g}\left(T_{2} / T_{2}^{\prime}\right) T_{2}^{\prime m}\right) *\left(g^{\prime}\left(T_{3} / T_{3}^{\prime}\right) T_{3}^{\prime 2}\right) .
\end{aligned}
$$

The generic fibre $X=\mathfrak{X}_{\eta}$ of $\mathfrak{X}$ is then geometrically connected and smooth over $K$, since it is everywhere locally étale isomorphic to $\mathbb{A}_{K}^{d}$, and defines a hypersurface in $\mathbb{P}_{K}^{\otimes(d+1)}, c f$. [Sat05] 33 , and [Fri83] for more general polynomials $G$.

Since $Q \times{ }_{k} Q=\operatorname{Spec}\left(h \otimes_{k} h\right)$ consists of $m Q$-rational points, we get in the obvious way that

- $Y^{[0]}$ consists of 2 Q-rational and 2 k-rational irreducible components:

$$
\begin{aligned}
& Y_{1}=Q \times \mathbb{P}_{k}^{1} \times \mathbb{P}_{k}^{1} \times \mathbb{P}_{k}^{\otimes(d-2)} \\
& Y_{2}=\mathbb{P}_{k}^{1} \times Q \times \mathbb{P}_{k}^{1} \times \mathbb{P}_{k}^{\otimes(d-2)} \\
& Y_{3}=\mathbb{P}_{k}^{1} \times \mathbb{P}_{k}^{1} \times O \times \mathbb{P}_{k}^{\otimes(d-2)} \\
& Y_{4}=\mathbb{P}_{k}^{1} \times \mathbb{P}_{k}^{1} \times \infty \times \mathbb{P}_{k}^{\otimes(d-2)}
\end{aligned}
$$

- $Y^{[1]}$ consists of $m+4 Q$-rational irreducible components:

$$
\begin{aligned}
& Y_{1} \cap Y_{2}=Q \times Q \times \mathbb{P}_{k}^{1} \times \mathbb{P}_{k}^{\otimes(d-2)}, \\
& Y_{1} \cap Y_{3}=Q \times \mathbb{P}_{k}^{1} \times O \times \mathbb{P}_{k}^{\otimes(d-2)}, \\
& Y_{1} \cap Y_{4}=Q \times \mathbb{P}_{k}^{1} \times \infty \times \mathbb{P}_{k}^{\otimes(d-2)}, \\
& Y_{2} \cap Y_{3}=\mathbb{P}_{k}^{1} \times Q \times O \times \mathbb{P}_{k}^{\otimes(d-2)}, \\
& Y_{2} \cap Y_{4}=\mathbb{P}_{k}^{1} \times Q \times \infty \times \mathbb{P}_{k}^{\otimes(d-2)},
\end{aligned}
$$

- $Y^{[2]}$ consists of $2 m$ Q-rational irreducible components:

$$
\begin{aligned}
& Y_{1} \cap Y_{2} \cap Y_{3}=Q \times Q \times O \times \mathbb{P}_{k}^{\otimes(d-2)}, \\
& Y_{1} \cap Y_{2} \cap Y_{4}=Q \times Q \times \infty \times \mathbb{P}_{k}^{\otimes(d-2)},
\end{aligned}
$$


- there are no higher $r$-fold intersections.

From the exact sequence 1.3.3:

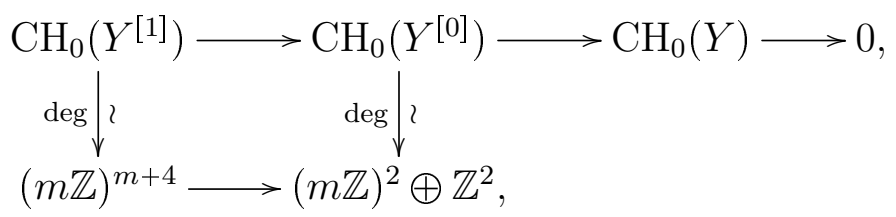

where the lower map is given by the matrix

$$
\left(\begin{array}{r|rrrrr} 
& Y_{12} & Y_{13} & Y_{14} & Y_{23} & Y_{24} \\
\hline Y_{1} & -m & -m & -m & 0 & 0 \\
Y_{2} & m & 0 & 0 & -m & -m \\
Y_{3} & 0 & m & 0 & m & 0 \\
Y_{4} & 0 & 0 & m & 0 & m
\end{array}\right),
$$

we get that

$$
\mathrm{CH}_{0}(Y) \cong \mathbb{Z} \oplus \mathbb{Z} / m
$$

cf. [Sat05] Lem. 3.4. Note, that we have the natural isomorphism from [Ful98] Thm. 3.3:

$$
\mathrm{CH}_{0}\left(Z \times{ }_{k} \mathbb{P}_{k}^{\otimes r}\right) \cong \mathrm{CH}_{0}(Z)
$$

The dual complex $\Gamma_{Y}$ of $Y$ is of the form

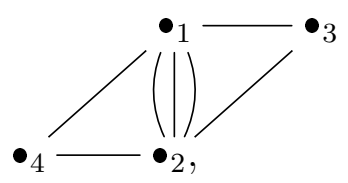

where every triangle is filled in. Therefore, it is connected and simply connected. So we get

$$
\begin{aligned}
& \mathrm{H}_{0}\left(\Gamma_{Y}, \mathbb{Z}\right) \cong \mathbb{Z}, \\
& \mathrm{H}_{1}\left(\Gamma_{Y}, \mathbb{Z}\right) \cong 0, \\
& \mathrm{H}_{2}\left(\Gamma_{Y}, \mathbb{Z}\right) \cong \mathbb{Z}^{m-1},
\end{aligned}
$$

where the rank of the second homology group can be computed by the fact, that the alternating sum of the ranks of the complex $\mathbb{Z}^{\pi_{0}\left(Y^{[\bullet]}\right)}$ :

$$
\mathbb{Z}^{2 m} \longrightarrow \mathbb{Z}^{m+4} \longrightarrow \mathbb{Z}^{4}
$$

coincide with the Euler-Poincaré characteristic. 
Now let $n$ be an arbitrary integer not divided by char $(k)$. Because $X$ is a smooth geometrically connected hypersurface defined by a multi-homogeneous polynomial, we get by 3.5 .5

$$
\mathrm{H}^{r}(\bar{X}, \mathbb{Z} / n) \cong \mathrm{H}^{r}\left(\mathbb{P}_{\bar{k}}^{\otimes(d+1)}, \mathbb{Z} / n\right) \cong \mathbb{Z} / n\left(-\frac{r}{2}\right)^{\left(\begin{array}{c}
d+1 \\
\frac{r}{2}
\end{array}\right)}
$$

for $0 \leqslant r \leqslant 2 \leqslant d-1$, since $d$ is chosen to be $\geqslant 3$. Like in 3.5.2 the Hochchild-Serre spectral sequence gives

$$
\# \mathrm{H}^{2}(X, \mathbb{Z} / n) \leqslant \# \mathrm{H}^{0}(\bar{X}) *\left(\# \mathrm{H}^{1}(\bar{X})\right)^{2} * \# \mathrm{H}^{2}(\bar{X})=n^{d+2} .
$$

If $\mathfrak{X}$ were regular, we would like in 3.5.2 have the exact sequence

$$
\mathrm{H}^{2}(X, \mathbb{Z} / n)^{\vee} \longrightarrow \mathrm{H}_{2}\left(\Gamma_{Y}, \mathbb{Z} / n\right) \longrightarrow \operatorname{ker} \rho_{n}^{X} \longrightarrow 0,
$$

leading to the estimations

$$
\begin{aligned}
\# \operatorname{ker} \rho_{n}^{X} & \geqslant \# \mathrm{H}_{2}\left(\Gamma_{Y}, \mathbb{Z} / n\right) / \# \mathrm{H}^{2}(X, \mathbb{Z} / n) \\
& \geqslant n^{m-1} / n^{d+2} \\
& =n^{m-d-3} \\
& \geqslant n^{3} \\
& >\# \mathrm{CH}_{0}(Y) / n \\
& \geqslant \# \operatorname{ker} \rho_{n}^{Y}
\end{aligned}
$$

for $d \geqslant 3$ and $m \geqslant d+6$. Taking the projective limit of the above sequence over $\mathbb{N}(\mathbb{L})$ for any set of prime numbers $\mathbb{L}$ not containing char $(k)$, we would get the similar result that

$$
\operatorname{rank}_{\mathbb{Z}_{\mathbb{L}}} \operatorname{ker} \rho_{\mathbb{L}}^{X} \geqslant 3
$$

observing that

$$
\lim _{n \in \mathbb{N}(\mathbb{L})} \mathrm{H}_{2}\left(\Gamma_{Y}, \mathbb{Z} / n\right)=\mathrm{H}_{2}\left(\Gamma_{Y}, \mathbb{Z}\right) \otimes \mathbb{Z}_{\mathbb{L}}=\mathbb{Z}_{\mathbb{L}}^{m-1}
$$

is free of rank $m-1$ and that

$$
\left(\lim _{n \in \mathbb{N}(\mathbb{L})} H^{r}(\bar{X}, \mathbb{Z} / n)\right)^{\vee}=\left(\underset{n \in \mathbb{N}(\mathbb{L})}{\lim } \mathbb{Z} / n\left(-\frac{r}{2}\right)^{\left(\begin{array}{c}
d+1 \\
\frac{r}{2}
\end{array}\right)}\right)^{\vee}=\left(\mathbb{Q} / \mathbb{Z}\left(-\frac{r}{2}\right)_{\mathbb{L} \text {-tors }}^{\left(\begin{array}{c}
d_{r}+1 \\
2
\end{array}\right)}\right)^{\vee}
$$

has $\mathbb{Z}_{\mathbb{L}}$-rank at most $\left(\begin{array}{c}d+1 \\ \frac{r}{2}\end{array}\right)$.

Example 3.5.8. Let the setting be like in 3.5 .7 and let $L \mid K$ be the unramified field extension corresponding to $h \mid k$ and $B \mid A$ the corresponding ring of integers. Now consider the base extension $\mathfrak{X}^{\prime}=\mathfrak{X}_{B}$. We then have $Y^{\prime}:=\mathfrak{X}_{s}^{\prime}=Y_{h}$ and $X^{\prime}:=\mathfrak{X}_{\eta}^{\prime}=X_{L}$. Since $X$ was smooth and geometrically integral, this also holds for $X^{\prime}$. Since $Q$ splits completely over $h$ we have that $Y_{1, h}$ and $Y_{2}, h$ each consists of $m$ irreducible components. So we get that 
- $Y^{[0]}$ consists of $2 m+2$ h-rational irreducible components:

$$
Y_{1}^{\prime i}, \quad Y_{2}^{\prime j}, \quad Y_{3}^{\prime}, \quad Y_{4}^{\prime},
$$

for $1 \leqslant i, j \leqslant m$,

- $Y^{{ }^{[1]}}$ consists of $m^{2}+4 m$ h-rational irreducible components:

$$
Y_{1}^{\prime i} \cap Y_{2}^{\prime j}, \quad Y_{1}^{\prime i} \cap Y_{3}^{\prime} . \quad Y_{1}^{i} \cap Y_{4}^{\prime}, \quad Y_{2}^{\prime j} \cap Y_{3}^{\prime} . \quad Y_{2}^{\prime j} \cap Y_{4}^{\prime} \text {, }
$$

for $1 \leqslant i, j \leqslant m$,

- $Y^{\prime[2]}$ consists of $2 m^{2}$ h-rational irreducible components:

$$
Y_{1}^{\prime i} \cap Y_{2}^{\prime j} \cap Y_{3}^{\prime}, \quad Y_{1}^{\prime i} \cap Y_{2}^{\prime i} \cap Y_{4}^{\prime},
$$

for $1 \leqslant i, j \leqslant m$,

- there are no higher $r$-fold intersections.

The dual complex to $Y^{\prime}$ is clearly connected and simply connected. So like before we get

$$
\begin{aligned}
& \mathrm{H}_{0}\left(\Gamma_{Y^{\prime}}, \mathbb{Z}\right) \cong \mathbb{Z}, \\
& \mathrm{H}_{1}\left(\Gamma_{Y^{\prime}}, \mathbb{Z}\right) \cong 0 \\
& \mathrm{H}_{2}\left(\Gamma_{Y^{\prime}}, \mathbb{Z}\right) \cong \mathbb{Z}^{m^{2}-2 m+1} .
\end{aligned}
$$

Since every component of a $Y^{\prime[r]}$ has a h-rational point, it is geometrically connected and since $\pi_{1}^{\mathrm{ab}}\left(\mathbb{P}_{k}^{\otimes r}\right) \cong G_{k}^{\mathrm{ab}}$ every component of $Y^{\prime}[0]$ is of "geometrically simply connected". Therefore by 1.4.11, the groups $\operatorname{ker} \rho^{Y}, \operatorname{ker} \rho_{n}^{Y}, \operatorname{ker} \rho_{\mathbb{L}}^{Y}$ vanish. Like before we would get that

$$
\# \operatorname{ker} \rho_{n}^{X} \geqslant n^{m^{2}-2 m-d-1}>0=\operatorname{ker} \rho_{n}^{Y}
$$

for $n$ not divided by $\operatorname{char}(h)$ and $m>d \geqslant 3$, and similar for the $\mathbb{Z}_{\mathbb{L}}$-rank of $\operatorname{ker} \rho_{\mathbb{L}}^{X}$, if $\mathfrak{X}_{B}$ were regular.

Remark 3.5.9. Note, that the examples 3.5.7 and 3.5.8 are not counterexamples to the question, if the kernels of the reciprocity maps modulo $n$ coincide, since the model $\mathfrak{X}$ in these examples is not regular, although its generic fibre is smooth and has strict semi-stable reduction. Moreover, these example show, that there can not be any regular hypersurface lift of $Y$.

If one now wants to resolve the singularities of $\mathfrak{X}$, then one would get new components in the special fibre, so that the new $\mathrm{H}_{2}\left(\Gamma, \mathbb{Z}_{\ell}\right)$ would have a smaller rank.

If one could construct a regular lift of $Y$, not necessarily as a hypersurface, then one has no control of $\mathrm{H}^{2}\left(X, \mathbb{Z}_{\ell}\right)$, which then would have a bigger rank.

Therefore, the construction of a counterexample is not easy and still is open. 


\section{Bibliography}

[AGV73] Michael Artin, Alexander Grothendieck, and Jean-Louis Verdier et. al, Théorie des Topos et Cohomologie Etale des Schemas, vol. 3, Lecture Notes in Mathematics, no. 305, Springer, Berlin, 1973.

[BBD82] Alexander Beilinson, Joseph Bernstein, and Pierre R. Deligne, Faisceaux pervers, Astérique 100 (1982), 3-171.

[BK86] Spencer Bloch and Kazuya Kato, p-adic etale cohomology, Publ. Math. I.H.E.S. 63 (1986), 107-152.

[Blo81] Spencer Bloch, Algebraic K-theory and class field theory for arithmetic surfaces, Ann. Math. 114 (1981), 229-265.

[Blo86] , Algebraic cycles and higher K-theory, Adv. in Math. 61 (1986), $267-304$.

[BO74] Spencer Bloch and Arthur Ogus, Gersten's conjecture and the homology of schemes, Ann. Scient. Éc. Norm. Sup. 4 (1974), no. 7, 181-202.

[CJS09] Vincent Cossart, Uwe Jannsen, and Shuji Saito, Canonical embedded and non-embedded resolution of singularities for excellent two-dimensional schemes, www.mathematik.uni-regensburg.de/Jannsen (2009), Preprint.

[CT93] Jean-Louis Colliot-Thélène, On the reciprocity sequence in higher class field theory of function fields, Algebraic K-Theory and Algebraic Topology, NATO ASI Series C, vol. 407, Kluwer Acad. Publ., Dordrecht, 1993, $35-55$.

[CTSS83] Jean-Louis Colliot-Thélène, Jean-Jacques Sansuc, and Christophe Soulé, Torsion dans le groupe de Chow de codimension deux, Duke Math. J. 50 (1983), 763-801.

[dJ96] Aise Johan de Jong, Smoothness, semi-stability and alterations, Pub. Math. IHES 83 (1996), 51-93.

[Fri83] Robert Friedman, Global smoothing of varieties with normal crossings, Ann. of. Math. 118 (1983), 75-114. 
[Fuj02] Kazuhiro Fujiwara, A proof of the absolute purity conjecture (after Gabber), Algebraic Geometry 2000, Azumino, Math. Soc. Japan, Tokyo, Adv. Stud. Pure Math. 36 (2002), 153-183.

[Ful98] William Fulton, Intersection theory, 2nd ed., Springer, Berlin, Heidelberg, 1998.

[GL01] Thomas Geißer and Marc Levine, The Bloch-Kato conjecture and a theorem of Suslin-Voevodsky., J. Reine Angew. Math. 530 (2001), 55-103.

[Gro71] Alexander Grothendieck, Revêtements Étales et Groupe Fondamental, Lecture Notes in Mathematics, vol. 224, Springer, Berlin, 1971, arXiv:math/0206203v2 [math.AG].

[Gro77] Alexander Grothendieck et. al, Cohomologie $\ell$-adique et Fonctions L, Lecture Notes in Mathematics, no. 589, Springer, Berlin, 1977.

[Har77] Robin Hartshorne, Algebraic Geometry, Graduate Texts in Mathematics, vol. 52, Springer, New York, 1977.

[Hu59] Sze-Tsen Hu, Homotopy theory, Academic Press, New York, London, 1959, 5th printing, 1971.

[HW09] Christian Haesemeyer and Chuck Weibel, Norm Varieties and the Chain Lemma (after Markus Rost), Algebraic Topology, Abel Symposium, no. 4, 95-130, Springer, Berlin, 2009.

[Ill79] Luc Illusie, Complex de De Rham-Witt et cohomologie cristalline, Ann. Scient. E.N.S. 12 (1979), 501-661.

[Ill09] , On Gabber's refined uniformization [Ga1], http://www.math.upsud.fr/ illusie/ (2009), Talks at the University of Tokyo 2008.

[Jan] Uwe Jannsen, Some cohomological considerations, unpublished notes to [JS03].

[Jan88] _ Continuous étale cohomology, Math. Ann. 280 (1988), 207-245.

[Jou83] Jean-Pierre Jouanolou, Théorémes de Bertini et Applications, Progress in Math., no. 42, Birkhäuser, Basel, 1983.

[JS03] Uwe Jannsen and Shuji Saito, Kato homology of arithmetic schemes and higher class field theory over local fields, Documenta Math., Extra volume: Kazuya Kato's Fiftieth Birthday (2003), 479-538. 
[JS09] _ Bertini theorems and Lefschetz pencils over discrete valuation rings, with applications to higher class field theory, arXiv:0911.1470v1 [math.AG] (2009), to appear in J. Algebraic Geometry.

[JSS09] Uwe Jannsen, Shuji Saito, and Kanetomo Sato, Étale duality for constructible sheaves on arithmetic schemes, arXiv:0910.3759v2 [math.AG] (2009).

[Kat86] Kazuya Kato, A Hasse principle for two dimensional global fields, J. Reine u. Angew. Math. 366 (1986), 142-183.

[KS83] Kazuya Kato and Shuji Saito, Unramified class field theory for arithmetic surfaces, Ann. Math. 118 (1983), 241-274.

[KS85] - Unramified class field theory of arithmetic schemes, Ann. Math. 118 (1985), 241-275.

[KS86] Global class field theory of arithmetic schemes, Contemp. Math., Amer. Math. Soc. 55 (1986), 255-331.

[KS09] Moritz Kerz and Alexander Schmidt, Covering data and higher dimensional class field theory, J. of Number Theory 129 (2009), 2569-2599.

[KS10] Moritz Kerz and Shuji Saito, Cohomological Hasse principle and motivic cohomology for arithmetic schemes, arXiv:1010.5930v1 [math.AG] (2010), Preprint.

[Lan56] Serge Lang, Unramified class field theory over function fields in several variables, Ann. Math. 64 (1956), 285-325.

[Liu02] Qing Liu, Algebraic Geometry and Arithmetic Curves, Oxford Graduate Texts in Mathematics, Oxford University Press, New York, 2002.

[Mil80] James S. Milne, Étale cohomology, Princeton University Press, Princeton, 1980 .

[Mil86] _ Values of zeta functions of varieties over finite fields, Amer. J. Math. 108 (1986), 297-360.

[Mil08] L Lectures on Etale Cohomology, 2.10 ed., http://www.jmilne.org/math/CourseNotes/lec.html, 2008, Course Notes.

[Mos99] Thomas Moser, A duality theorem for étale p-torsion sheaves on complete varieties over a finite field, Compositio Math. 117 (1999), 123-152. 
[MS83] Alexander S. Merkurjev and Andrei A. Suslin, K-cohomology of SeveriBrauer varieties and the norm residue homomorphism, Math. USSR Izvestiya 21 (1983), 307-340.

[MSA99] Kazuya Matsumi, Kanetomo Sato, and Masanori Asakura, On the kernel of the reciprocity map of normal surfaces over finite fields, K-Theory $\mathbf{1 8}$ (1999), 203-234.

[NSW08] Jürgen Neukirch, Alexander Schmidt, and Kay Wingberg, Cohomology of number fields, 2nd ed., Grundlehren der math. Wissenschaften, vol. 323, Springer, Berlin, Heidelberg, 2008.

[Poo04] Bjorn Poonen, Bertini theorems over finite fields, Ann. of Math. 160 (2004), no. 3, 1099-1127, arXiv:math/0204002v1 [math.AG].

[Ras95] Wayne Raskind, Abelian class field theory of arithmetic schemes, Proceedings of Symposia in Pure Mathematics 58 (1995), no. 1, 85-187.

[RZ82] Michael Rapoport and Thomas Zink, Über die lokale Zetafunktion von Shimuravarietäten. Monodromiefiltration und verschwindende Zyklen in ungleicher Charakteristik, Invent. math. 68 (1982), 21-101.

[Sai85a] Shuji Saito, Class field theory for curves over local fields, Journal of Number Theory 21 (1985), 44-80.

[Sai85b] _ Unramified class field theory of arithmetical schemes, Ann. Math. 121 (1985), 251-281.

[Sai98] _ A global duality theorem for varieties over global fields, Algebraic $K$-theory: connections with geometry and topology (1998), 425-444, Kluwer Academic Publishers.

[Sat05] Kanetomo Sato, Non-divisible cycles on surfaces over local fields, Journal of Number Theory 114 (2005), 272-297.

[Ser02] Jean-Pierre Serre, Galois cohomology, corr. 2nd ed., Springer monographs in mathematics, Springer, Berlin, Heidelberg, 2002.

[SJ06] Andrei Suslin and Seva Joukhovitski, Norm varieties, J. Pure Appl. Algebra 206 (2006), no. 1-2, 245-276.

[Sti06] Jakob Stix, A general Seifert-Van Kampen theorem for algebraic fundamental groups, Publications of RIMS 42 (2006), no. 3, 763-786.

[Sug09] Rin Sugiyama, On the kernel of the recipocity map of simple normal crossing varieties over finite fields, Preprint (2009). 
[Suw95] Noriyuki Suwa, A note on Gersten's conjecture for logarithmic Hodge-Witt sheaves, K-Theory 9 (1995), 245-271.

[SV00] Andrei Suslin and Vladimir Voevodsky, Bloch-Kato conjecture and motivic cohomology with finite coefficients, NATO Sci. Ser. C Math. Phys. Sci., 548, Kluwer Acad. Publ., Dordrecht, 2000, The arithmetic and geometry of algebraic cycles (Banff, AB, 1998), 117-189.

[Sza99] Tamás Szamuely, Sur l'application de réeciprocité pour une surface fibrée en coniques définie sur un corps local, K-Theory 18 (1999), 173-179.

[Sza00] _ Sur la théorie des corps de classes pour les variétés sur les corps p-adiques, J. Reine Angew. Math. 525 (2000), 183-212.

[Sza09] _ Galois groups and fundamental groups, Cambridge Studies in Advanced Mathematics, vol. 117, Cambridge University Press, Cambridge, New York, 2009.

[Voe10a] Vladimir Voevodsky, Motivic Eilenberg-MacLane spaces, arXiv:0805.4432v3 [math.AG] (2010), to appear in Publ. IHES.

[Voe10b] _ On motivic cohomology with $\mathbb{Z} / \ell$-coefficients, arXiv:0805.4430v2 [math.AG] (2010), to appear in Annals of Math.

[Wei95] Charles A. Weibel, An Introduction to Homological Algebra, paperback ed., Cambridge Studies in Advanced Mathematics, no. 38, Cambridge University Press, Cambridge, New York, 1995.

[Wie06] Götz Wiesend, A construction of covers of arithmetic schemes, J. Number Theory 121 (2006), no. 1, 118-131.

[Wie07] _ Class field theory for arithmetic schemes, Math. Zeitschriften 256 (2007), no. 4, 717-729.

[Yos03] Teruyoshi Yoshida, Finiteness theorems in the class field theory of varieties over local fields, J. Number Theory 101 (2003), no. 1, 138-150. 